
Outfalls, June-August 1996

by

W. L. Specht

Westinghouse Savannah River Company

Savannah River Site

Aiken, South Carolina 29808

This paper was prepared in connection with work done under the above contract number with the U. S. Department of Energy. By acceptance of this paper, the publisher and/or recipient acknowledges the U. S. Government's right to retain a nonexclusive, royalty-free license in and to any copyright covering this paper, along with the right to reproduce and to authorize others to reproduce all or part of the copyrighted paper. 


\section{DISCLAIMER}

This report was prepared as an account of work sponsored by an agency of the United States Government. Neither the United States Government nor any agency thereof, nor any of their employees, makes any warranty, express or implied, or assumes any legal liability or responsibility for the accuracy, completeness, or usefulness of any information, apparatus, product, or process disclosed, or represents that its use would not infringe privately owned rights. Reference herein to any specific commercial product, process, or service by trade name, trademark, manufacturer, or otherwise does not necessarily constitute or imply its endorsement, recommendation, or favoring by the United States Government or any agency thereof. The views and opinions of authors expressed herein do not necessarily state or reflect those of the United States Government or any agency thereof.

This report has been reproduced directly from the best available copy.

Available to DOE and DOE contractors from the Office of Scientific and Technical Information, P.O. Box 62, Oak Ridge, TN 37831; prices available from (615) 576-8401.

Available to the public from the National Technical Information Service, U.S. Department of Commerce, 5285 Port Royal Road, Springfield, VA 22161. 



\section{DISCLAIMER}

This report was prepared as an account of work sponsored by an agency of the United States Government. Neither the United States Government nor any agency thereof, nor any of their employees, make any warranty, express or implied, or assumes any legal liability or responsibility for the accuracy, completeness, or usefulness of any information, apparatus, product, or process disclosed, or represents that its use would not infringe privately owned rights. Reference herein to any specific commercial product, process, or service by trade name, trademark, manufacturer, or otherwise does not necessarily constitute or imply its endorsement, recommendation, or favoring by the United States Government or any agency thereof. The views and opinions of authors expressed herein do not necessarily state or reflect those of the United States Government or any agency thereof. 


\section{DISCLAMMER}

Portions of this document may be illegible in electronic image produets. Images are produced from the best available original document. 


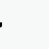


October 03, 1996

TO: $\quad$ Mike Boerste, $773-43 \mathrm{~A}$

FROM: Winona L. Specht, 773-42A

Re: Final Report for A-Area Toxicity Tests

Attached is the final report which summarizes the results of the toxicity tests that were conducted on A-Area discharges, under Work Authorization Document LT960170.

cc: $\quad$ N. Turner, $773-43 A$

J.B. Gladden, 773-42A

J.L. Keyes, 742-A

D.V. Osteen, 742-A

W. Payne, 742-A

T. Smith, 742-A

L. Eldridge, 742-A

C.R. Whitehurst, 763-A

W. Wilmarth, 773-43A

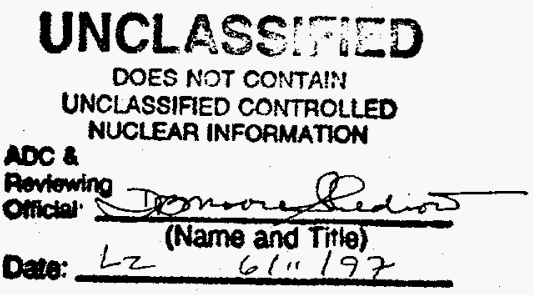




\title{
RESULTS OF CHRONIC TOXICITY TESTS CONDUCTED ON \\ SELECTED A-AREA OUTFALLS, JUNE-AUGUST 1996
}

\author{
by Winona L. Specht, ESS/SRTC
}

\subsection{Introduction}

In anticipation of possible toxicity testing requirements in the SRS's new (1996) NPDES permit, toxicity tests were performed at selected A-Area NPDES outfalls in order to determine if the outfalls were toxic. Chronic definitive toxicity tests were conducted on Ceriodaphnia dubia using water collected from nine locations (Table 1) during the summer of 1996.

Table 1. Sampling Locations for Toxicity Testing, June-August 1996.

Location Source Inputs to Outfall

A-001A Air Stripper

A-001B Lab drains, neutralization facility

A-001C Non-contact cooling water, steam condensate, storm water

A-003 Chilled water, steam condensate, cooling tower overflow, storm water

A-004 Steam condensate, storm water

A-005 Greenhouse water, A/C condensate, steam cleaning rack, lab drains, animal holding pen water, and storm water.

A-006 Overflow from animal holding ponds, storm water

AT-001 Combined discharge of A-001, A-003, A-004, and A-005

WE-001 Approximately $1 \mathrm{~km}$ downstream from AT-001; includes A-006

\subsection{Methods}

Definitive chronic toxicity tests were conducted on Ceriodaphnia dubia using EPA methods (Weber et al. 1989). Test conditions are specified in Table 2. Water collected from Upper Three Runs at Road 8-1 (Treadway Bridge) was used as control water and dilution water for the tests. Toxicity tests were performed on a series of dilutions, $(0,6.25 \%, 12.5 \%, 25 \%, 50 \%$ and $100 \%$ sample water $)$, using 10 test organisms per concentration. At the end of the 7-day test the reproductive success (number of young produced) of organisms exposed to each dilution was compared statistically to that the control group $(0 \%)$ in order to determine if reproduction was impaired.

The results of the tests were reported as NOEC's (No Observed Effect Concentration), which was the highest concentration tested that did not cause a significant reduction in reproduction. If there was no reproductive impairment, the NOEC was $100 \%$. In addition, any mortality that occurred during the test was also recorded. If an outfall was found to be non-toxic, a second chronic definitive test was performed several weeks later to confirm that the outfall was consistently not toxic. Water quality parameters routinely measured during the toxicity tests include temperature, $\mathrm{pH}$, hardness, alkalinity and specific conductance. 
Table 2. Test Conditions for Conducting a 7-day Definitive Water

Toxicity Test with Ceriodaphnia dubia

\begin{tabular}{ll} 
Parameter & Conditions \\
\hline Test type & Static renewal \\
Temperature & $25 \pm 1^{\circ} \mathrm{C}$ \\
Light quality & Ambient laboratory illumination \\
Light intensity & $50-100$ foot candles \\
Photoperiod & $16 \mathrm{~L}: 8 \mathrm{D}$ \\
Test chamber size & $30 \mathrm{ml}$ \\
Test solution volume & $15 \mathrm{ml}$ \\
Renewal of test water & Daily \\
Age of test organisms & Less than $24 \mathrm{~h}$ at start of test; all released \\
& within a $12 \mathrm{~h}$ period \\
\# neonates/test chamber & 1 \\
\# Neonates/concentration & 10 \\
Feeding regime & $0.1 \mathrm{ml}$ YCT and algal suspension/test chamber/day \\
Aeration & None \\
Dilution water & Upper Three Runs at Road $8-1$ \\
Number of dilutions & 5 and a control \\
dilution factor & 0.5 \\
Test duration & Until at least $60 \%$ of control females have three \\
& broods (usually 7 days) \\
Test endpoints & Survival and reproduction \\
Test acceptability & $80 \%$ or greater survival and an average of at least \\
& 15 young/surviving female in the control solutions \\
Sampling requirements & A minimum of three samples are collected for the \\
& seven day test (usually on days 1,3, and 5 of the \\
& test). Samples are less than 72 hours old when \\
& used.
\end{tabular}

\subsection{Results}

The No Observed Effect Concentrations (NOEC's) for the toxicity tests are presented in Table 3. Copies of the toxicity testing reports can be found in the Appendix. Of the nine locations that were tested, three of the locations were not toxic (A-001A, A-006, and WE-001). The remaining six locations had NOEC's ranging from $<6.25 \%$ to $50 \%$ effluent. The most toxic sampling locations were A-001C and A-003, which both exhibited reproductive impairment at the lowest effluent concentration that was tested $(6.25 \%)$. All of the six locations that were chronically toxic were also acutely toxic when undiluted, producing $100 \%$ mortality in undiluted (100\%) effluent (Table 3 ). 
Table 3. No Observed Effect Concentrations (NOEC's) and Mortality for Toxicity Tests Performed on A-Area Outfalls, June-August 1996

$\begin{array}{lrcr}\text { Location } & \text { Test Date } & \text { NOEC } & \begin{array}{r}\text { Percent Mortality } \\ \text { in 100\% Efflue }\end{array} \\ \text { A-001A } & & & 20 \% \\ \text { A-001A (repeat) } & 7 / 16 / 96 & 100 \% & 0 \% \\ \text { A-001B } & 8 / 6 / 96 & 100 \% & 100 \% \\ \text { A-001C } & 6 / 25 / 96 & 12.5 \% & 100 \% \\ \text { A-003 } & 6 / 25 / 96 & <6.25 \% & 100 \% \\ \text { A-004 } & 6 / 25 / 96 & <6.25 \% & 100 \% \\ \text { A-005 } & 7 / 9 / 96 & 25 \% & 100 \% \\ \text { A-006 } & 7 / 9 / 96 & 50 \% & 0 \% \\ \text { A-006 (repeat) } & 7 / 9 / 96 & 100 \% & 0 \% \\ \text { AT-001 } & 7 / 23 / 96 & 100 \% & 100 \% \\ \text { WE-001 } & 7 / 16 / 96 & 25 \% & 0 \% \\ \text { WE-001 (repeat) } & 7 / 16 / 96 & 100 \% & 0 \% \\ & 7 / 23 / 96 & 100 \% & \end{array}$

${ }^{a} 100 \%$ mortality in $25 \%$ effluent

b $100 \%$ mortality in $12.5 \%$ effluent

\subsection{Summary/Conclusions/Path Forward}

Chronic definitive toxicity tests were conducted at nine locations in the vicinity of A-Area. Six of the nine locations were toxic, with NOEC's ranging from $<6.25 \%$ to $50 \%$. Sampling location AT-001 which located downstream of outfalls A-001 (which includes A-001A, A-001B, and A-001C), A-003, A-004, and A-005 was toxic, with an NOEC of $25 \%$. However, sampling location WE-001, which is located approximately $1 \mathrm{~km}$ downstream from AT-001, at the downstream end of a small wetland, was not toxic. Since there are no major tributaries flowing into Tim's Branch between these two sampling points, these results suggest that toxicants present at AT-001 are being removed by the wetland.

In order to determine why six of the sampling locations were toxic, Toxicity Identification Evaluations (TIE's) must be performed. TIE's were initiated in August 1996 at each of these six locations. 


\section{References}

Weber, C.I, W.H. Peltier, T.J. Norberg-King, W.B. Horning II, F.A. Kessler, J.R. Menkedick, T.W. Neilheisel, P.A. Lewis, D.J. Klemm, Q.H. Pickering, E.L. Robinson, J.M. Lazorchak, L.J. Wymer, and R.W. Freyberg. 1989. Short-term Methods for Estimating the Chronic Toxicity of Effluents and Receiving Waters to Freshwater Organisms. EPA/600/4-89/001. 
APPENDIX

* 
(864) 877-6942 - FAX (864) 877-6938

P.O. Box 16414, Greenville, SC 29606 - 4 Craftsman Court, Greer, SC 29650

\section{Day Chronic Definitive Survival and Reproduction Bioassay}

Method: EPA/600/4-89/001

Test Organism: Ceriodaphnia dubia

Facility: Westinghouse Savannah River Company

Sample ID: A-001B

Date: $\quad 6 / 25 / 96$ 


\section{CHRONIC DEFINITIVE SURVIVAL AND REPRODUCTION TEST Survival and Reproduction Results}

Client: Westinghouse Savannah River Company

Sample Identification: A-001B

Test. Date: $\quad$ 6/25/96

T5971

\begin{tabular}{|c|c|c|c|c|c|c|}
\hline \multicolumn{2}{|c|}{ Reproduction at } & \multicolumn{3}{c|}{$0 \%$} & \multicolumn{3}{c|}{ Concentration } \\
\hline Rep. & Day 3/4 & Day 5 & Day 6 & Day 7 & Day 8 & Total \\
\hline 1 & 0 & 3 & 9 & 0 & 0 & 12 \\
\hline 2 & 1 & 2 & 0 & 10 & 10 & 23 \\
\hline 3 & 0 & 3 & 10 & 0 & 9 & 22 \\
\hline 4 & 3 & 6 & 7 & 0 & 11 & 27 \\
\hline 5 & 3 & 4 & 4 & 0 & 13 & 24 \\
\hline 6 & 0 & 6 & 0 & 15 & 0 & 21 \\
\hline 7 & 3 & 7 & 10 & 0 & 0 & 20 \\
\hline 8 & 0 & 4 & 0 & 5 & 0 & 9 \\
\hline 9 & 2 & 6 & 9 & 0 & 0 & 17 \\
\hline 10 & 3 & 0 & 0 & 2 & 13 & 18 \\
\hline
\end{tabular}

\begin{tabular}{|c|c|c|c|c|c|c|}
\hline \multicolumn{2}{|c|}{ Reproduction at } & $6.25 \%$ & \multicolumn{3}{c|}{ Concentration } \\
\hline Rep. & Day 3/4 & Day 5 & Day 6 & Day 7 & Day 8 & Total \\
\hline 1 & 0 & L & L & L & L & L \\
\hline 2 & 0 & 0 & 0 & 6 & 11 & 17 \\
\hline 3 & 0 & 7 & 0 & 0 & 12 & 19 \\
\hline 4 & 0 & 0 & 0 & 0 & 13 & 13 \\
\hline 5 & 0 & 0 & 5 & 0 & 13 & 18 \\
\hline 6 & 0 & 0 & 7 & 0 & 2 & 9 \\
\hline 7 & 3 & 6 & 6 & 0 & 0 & 15 \\
\hline 8 & 0 & 0 & 0 & 4 & 13 & 17 \\
\hline 9 & 2 & 0 & 0 & 0 & 13 & 15 \\
\hline 10 & 3 & 0 & 0 & 0 & 6 & 9 \\
\hline
\end{tabular}

\begin{tabular}{|c|c|c|c|c|c|c|}
\hline \multicolumn{2}{|c|}{ Reproduction at } & \multicolumn{3}{c|}{$12.5 \%$} & \multicolumn{3}{c|}{ Concentration } \\
\hline Rep. & Day 3/4 & Day 5 & Day 6 & Day 7 & Day 8 & Total \\
\hline 1 & 4 & 3 & 0 & 8 & 0 & 15 \\
\hline 2 & 0 & 0 & 0 & 10 & 10 & 20 \\
\hline 3 & 0 & 0 & 0 & 0 & 0 & 0 \\
\hline 4 & 4 & 0 & 0 & 8 & 13 & 25 \\
\hline 5 & 3 & 2 & 0 & 0 & 12 & 17 \\
\hline 6 & 3 & 0 & 4 & 7 & 0 & 14 \\
\hline 7 & 4 & 0 & 1 & 0 & 9 & 14 \\
\hline 8 & 0 & 1 & 0 & 0 & 12 & 13 \\
\hline 9 & 3 & 0 & 2 & 0 & 10 & 15 \\
\hline 10 & 4 & 0 & 0 & 0 & 7 & 11 \\
\hline
\end{tabular}

\begin{tabular}{|c|c|c|c|c|c|c|}
\hline \multicolumn{3}{|c|}{ Reproduction at } & \multicolumn{5}{c|}{$25.0 \%$} & \multicolumn{3}{c|}{ Concentration } \\
\hline Rep. & Day 3/4 & Day 5 & Day 6 & Day 7 & Day 8 & Total \\
\hline 1 & 1 & 5 & 0 & 11 & 0 & 17 \\
\hline 2 & 0 & 0 & 1 & 9 & 0 & 10 \\
\hline 3 & 3 & 0 & 0 & 2 & 13 & 18 \\
\hline 4 & $X$ & $X$ & $X$ & $X$ & $X$ & 0 \\
\hline 5 & 2 & 5 & 1 & 0 & 8 & 16 \\
\hline 6 & 1 & 0 & 3 & 0 & 14 & 18 \\
\hline 7 & 3 & 2 & 0 & 0 & 11 & 16 \\
\hline 8 & 0 & 0 & 0 & 0 & 11 & 11 \\
\hline 9 & 4 & 2 & 0 & 0 & 12 & 18 \\
\hline 10 & 0 & 3 & 0 & 0 & 13 & 16 \\
\hline
\end{tabular}

\begin{tabular}{|c|c|c|c|c|c|c|}
\hline \multicolumn{3}{|c|}{ Reproduction at } & \multirow{2}{*}{$\frac{50.0 \%}{\text { Day } 6}$} & \multicolumn{3}{|c|}{ Concentration } \\
\hline Rep. & Day 3/4 & Day 5 & & Day 7 & Day 8 & Total \\
\hline 1 & 3 & $x$ & $\mathrm{X}$ & $\mathrm{X}$ & $X$ & 3 \\
\hline 2 & $\bar{X}$ & $\mathrm{X}$ & $\bar{X}$ & $\mathrm{X}$ & $\mathrm{X}$ & 0 \\
\hline 3 & $\bar{X}$ & $\mathrm{X}$ & $\bar{X}$ & $\mathrm{X}$ & $\mathrm{X}$ & 0 \\
\hline 4 & $X$ & $\mathrm{X}$ & $\mathrm{X}$ & $x$ & $\mathrm{X}$ & 0 \\
\hline 5 & $\bar{x}$ & $x$ & $x$ & $x$ & $\mathrm{X}$ & 0 \\
\hline 6 & 0 & 5 & $x$ & $\bar{X}$ & $\mathrm{X}$ & 5 \\
\hline 7 & $X$ & $x$ & $x$ & $X$ & $x$ & 0 \\
\hline 8 & $x$ & $X$ & $X$ & $\mathrm{X}$ & $X$ & 0 \\
\hline 9 & $x$ & $X$ & $x$ & $x$ & $x$ & 0 \\
\hline 10 & 0 & $\mathrm{X}$ & $\mathrm{X}$ & $x$ & $\mathrm{X}$ & 0 \\
\hline
\end{tabular}

\begin{tabular}{|c|l|l|l|l|l|l|}
\hline \multicolumn{2}{|l}{ Reproduction at } & \multicolumn{1}{l|}{$100 \%$} & \multicolumn{3}{l|}{ Concentration } \\
\hline Rep. & Day 3/4 & Day 5 & Day 6 & Day 7 & Day 8 & Total \\
\hline 1 & $\mathrm{X}$ & $\mathrm{X}$ & $\mathrm{X}$ & $\mathrm{X}$ & $\mathrm{X}$ & 0 \\
\hline 2 & $\mathrm{X}$ & $\mathrm{X}$ & $\mathrm{X}$ & $\mathrm{X}$ & $\mathrm{X}$ & 0 \\
\hline 3 & $\mathrm{X}$ & $\mathrm{X}$ & $\mathrm{X}$ & $\mathrm{X}$ & $\mathrm{X}$ & 0 \\
\hline 4 & $\mathrm{X}$ & $\mathrm{X}$ & $\mathrm{X}$ & $\mathrm{X}$ & $\mathrm{X}$ & 0 \\
\hline 5 & $\mathrm{X}$ & $\mathrm{X}$ & $\mathrm{X}$ & $\mathrm{X}$ & $\mathrm{X}$ & 0 \\
\hline 6 & $\mathrm{X}$ & $\mathrm{X}$ & $\mathrm{X}$ & $\mathrm{X}$ & $\mathrm{X}$ & 0 \\
\hline 7 & $\mathrm{X}$ & $\mathrm{X}$ & $\mathrm{X}$ & $\mathrm{X}$ & $\mathrm{X}$ & 0 \\
\hline 8 & $\mathrm{X}$ & $\mathrm{X}$ & $\mathrm{X}$ & $\mathrm{X}$ & $\mathrm{X}$ & 0 \\
\hline 9 & $\mathrm{X}$ & $\mathrm{X}$ & $\mathrm{X}$ & $\mathrm{X}$ & $\mathrm{X}$ & 0 \\
\hline 10 & $\mathrm{X}$ & $\mathrm{X}$ & $\mathrm{X}$ & $\mathrm{X}$ & $\mathrm{X}$ & 0 \\
\hline
\end{tabular}

$X=$ Dead

$L=$ Lost or caught in surface tension of water 


\section{CHRONIC DEFINITIVE SURVIVAL AND REPRODUCTION TEST \\ Statistical Analyses}

Client: Westinghouse Savannah River Company

Sample Identification: A-001B

Test Date: $\quad$ 6/25/96

\begin{tabular}{|lrr|}
\hline Test for Normality & & \\
Test Used: Shapiro Wilks & W= & 0.906 \\
& critical & 0.93 \\
& & \\
The data are not normal in distribution \\
\end{tabular}

\begin{tabular}{|lrr|}
\hline Test for Homogeneity of Variance & \\
Test Used: Bartlett's Test & $\mathrm{B}=$ & 1.98 \\
& critical & 13.28 \\
The data are homogeneous in variance
\end{tabular}

\begin{tabular}{|c|c|c|c|c|c|}
\hline \multicolumn{6}{|c|}{ Test for Differences in Reproduction } \\
\hline \multicolumn{2}{|l|}{ Test Tyne Used. } & \multicolumn{4}{|c|}{ Wilcoxon's Test } \\
\hline$t$ Test & \multicolumn{5}{|c|}{ Test Concentration } \\
\hline Control & $6.25 \%$ & $12.5 \%$ & $25 \%$ & $50.0 \%$ & $100 \%$ \\
\hline t Value & 2.04 & 2.22 & 2.40 & 8.39 & N/A \\
\hline critical & 2.33 & 2.33 & 2.33 & 2.33 & \\
\hline mean & 14.7 & 14.4 & 14.0 & 0.8 & 0.0 \\
\hline \multicolumn{6}{|l|}{ MSD $=$} \\
\hline \multicolumn{2}{|l|}{ Wilcoxon's Test } & \multicolumn{4}{|c|}{ Test Concentration } \\
\hline & $6.25 \%$ & $12.5 \%$ & $25 \%$ & $50.0 \%$ & $100 \%$ \\
\hline Rank Sum & & 84 & 76 & $\mathrm{~N} / \mathrm{A}$ & N/A \\
\hline critical & & 76 & 76 & & \\
\hline \multicolumn{6}{|c|}{$\begin{array}{l}\text { LOEC }=25 \% \text { effluent } \\
\text { NOEC }=12.5 \% \text { effluent }\end{array}$} \\
\hline
\end{tabular}

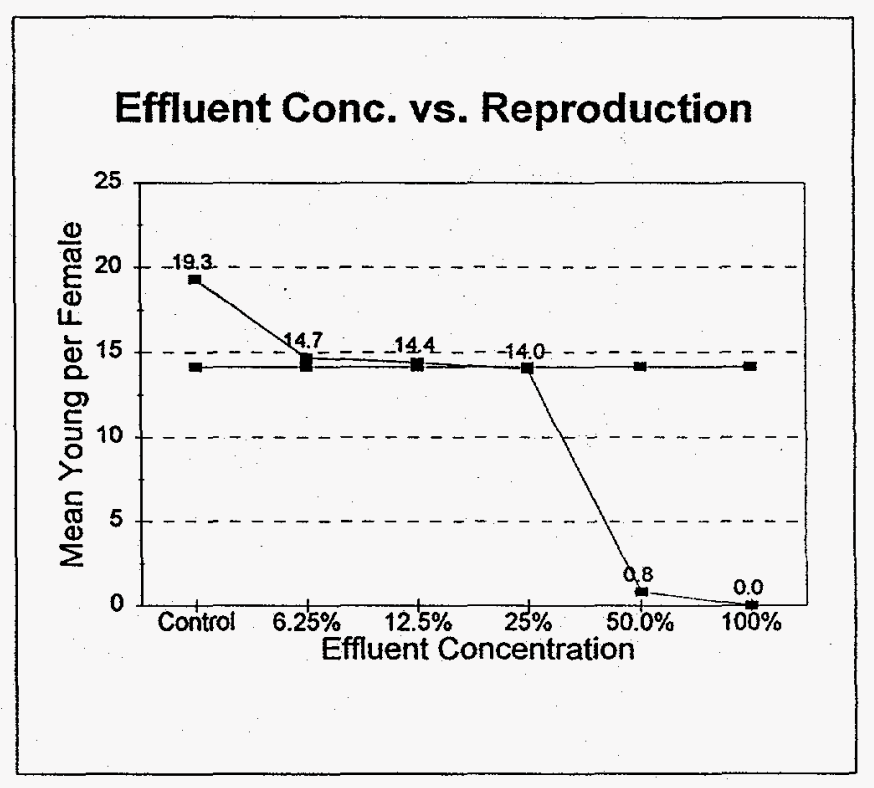

\section{Summary}

The effuent was marginally non-toxic at $6.25-12.5 \%$ concentration and marginally toxic at $25 \%$ concentration. 


\section{CHRONIC DEFINITIVE SURVIVAL AND REPRODUCTION TEST Water Chemistry}

Client: Westinghouse Savannah River Company

Sample Identification: A-001B

Test Date: $\quad$ 6/25/96 T5971

pH (After Renewal)

\begin{tabular}{|l|r|r|r|r|r|r|}
\hline \multicolumn{7}{|c|}{ Test Concentration } \\
\hline & Control & $6.25 \%$ & $12.5 \%$ & $25 \%$ & $50.0 \%$ & $100 \%$ \\
\hline Initial & 7.86 & 7.40 & 7.36 & 7.40 & 7.49 & 7.81 \\
\hline Day 1 & 7.42 & 7.43 & 7.40 & 7.39 & 7.42 & \\
\hline Day 2 & 7.76 & 8.00 & 7.93 & 7.89 & 7.86 & \\
\hline Day 3 & 7.00 & 6.92 & 6.98 & 7.06 & 7.15 & \\
\hline Day 4 & & 7.35 & 7.33 & 7.33 & 7.28 & \\
\hline Day 5 & & 7.53 & 7.56 & 7.55 & 7.56 & \\
\hline Day 6 & & 7.61 & 7.58 & 7.56 & & \\
\hline
\end{tabular}

\section{Dissolved Oxygen (After Renewal)}

\begin{tabular}{|l|r|r|r|r|r|r|}
\hline \multicolumn{7}{|c|}{ Test Concentration } \\
\hline & Control & $6.25 \%$ & $12.5 \%$ & $25 \%$ & $50.0 \%$ & $100 \%$ \\
\hline Initial & 9.1 & 8.7 & 8.6 & 8.7 & 8.7 & 8.7 \\
\hline Day 1 & 9.1 & 8.4 & 8.5 & 8.5 & 8.5 & \\
\hline Day 2 & & 8.8 & 8.8 & 8.7 & 8.7 & \\
\hline Day 3 & 8.8 & 8.8 & 8.8 & 8.7 & 8.7 & \\
\hline Day 4 & 8.8 & 8.7 & 8.8 & 8.8 & 8.9 & \\
\hline Day 5 & & 8.7 & 8.7 & 8.7 & 8.7 & \\
\hline Day 6 & & 8.6 & 8.6 & 8.6 & & \\
\hline
\end{tabular}

Dilution Water \& Effluent Parameters

\begin{tabular}{|l|c|r|r|r|l|}
\hline & Res Cl. & Alkalin & Hard. & Cond. & \\
\hline & $\mathrm{mg} / \mathrm{L}$ & $\mathrm{mg} / \mathrm{L}$ & $\mathrm{mg} / \mathrm{L}$ & $\mu \mathrm{mhos}$ & \\
\hline Eff. Sample 1 & $<0.01$ & 59.7 & 14 & 127.3 & \\
\hline Eff. Sample 2 & $<.01$ & 49.4 & 20 & 125.3 & \\
\hline Eff. Sample 3 & 0.07 & 39.1 & 8 & 112.4 & \\
\hline & & & & & \\
\hline Dilution Water & & 70 & 84 & 187.9 & \\
\hline & & & & & \\
\hline & & & & & \\
\hline
\end{tabular}

pH (Before Renewal)

\begin{tabular}{|l|r|r|r|r|r|r|}
\hline \multicolumn{7}{|c|}{ Test Concentration } \\
\hline & Control & $6.25 \%$ & $12.5 \%$ & $25 \%$ & $50.0 \%$ & $100 \%$ \\
\hline Day 1 & 7.18 & 7.29 & 7.33 & 7.38 & 7.31 & 7.38 \\
\hline Say 2 & 7.86 & 7.64 & 7.61 & 7.61 & 7.60 & \\
\hline Day 3 & 7.16 & 7.24 & 7.30 & 7.44 & 7.61 & \\
\hline Day 4 & & 8.10 & 7.97 & 7.94 & 7.83 & \\
\hline Day 5 & & 7.32 & 7.36 & 7.37 & 7.31 & \\
\hline Day 6 & & 7.66 & 7.62 & 7.61 & & \\
\hline Final & & & & & & \\
\hline
\end{tabular}

Dissolved Oxygen (Before Renewal)

\begin{tabular}{|l|r|r|r|r|r|r|}
\hline \multicolumn{7}{|c|}{ Test Concentration } \\
\hline & Control & $6.25 \%$ & $12.5 \%$ & $25 \%$ & $50.0 \%$ & $100 \%$ \\
\hline Day 1 & & 8.5 & 8.5 & 8.4 & 8.4 & 8.4 \\
\hline Day 2 & & 8.4 & 8.4 & 8.4 & 8.4 & \\
\hline Day 3 & & 8.3 & 8.3 & 8.4 & 8.4 & \\
\hline Day 4 & & 8.4 & 8.6 & 8.4 & 8.4 & \\
\hline Day 5 & & 8.5 & 8.6 & 8.6 & 8.6 & \\
\hline Day 6 & & 8.5 & 8.5 & 8.5 & & \\
\hline Final & & & & & & \\
\hline
\end{tabular}

Temperature
\begin{tabular}{|l|r|}
\hline Incubator ${ }^{\circ} \mathrm{C}$ \\
\hline Initial & 24.6 \\
\hline Day 1 & 24.6 \\
\hline Day 2 & 24.9 \\
\hline Day 3 & 24.1 \\
\hline Day 4 & 25.7 \\
\hline Day 5 & 24.7 \\
\hline Day 6 & 25.2 \\
\hline Final & 24.6 \\
\hline
\end{tabular}




\section{Day Chronic Definitive Survival and Reproduction Bioassay \\ Method: EPA/600/4-89/001}

Test Organism:

Ceriodaphnia dubia

Facility:Westinghouse Savannah River Company

Sample ID:T6097 (A-001A)

Date: $\quad 7-16-96$ 


\section{CHRONIC DEFINITIVE SURVIVAL AND REPRODUCTION TEST Survival and Reproduction Results}

Client: Westinghouse Savannah River Company

Sample Identification: T6097 (A-001A)

Test Date: $\quad$ 7-16-96

\begin{tabular}{|c|c|c|c|c|c|c|c|}
\hline \multicolumn{1}{|c|}{ Reproduction at } & $0.0 \%$ & \multicolumn{1}{c|}{ Concentration } \\
\hline Rep. & Day 2/3 & Day 4 & Day 5 & Day 6 & Day 7 & Day 8 & Total \\
\hline 1 & 0 & 4 & 8 & 0 & 10 & 0 & 22 \\
\hline 2 & 0 & 2 & 7 & 0 & 0 & 8 & 17 \\
\hline 3 & 0 & 5 & 7 & 0 & 11 & 0 & 23 \\
\hline 4 & 0 & $\mathrm{~L}$ & $\mathrm{~L}$ & $\mathrm{~L}$ & $\mathrm{~L}$ & $\mathrm{~L}$ & $\mathrm{~L}$ \\
\hline 5 & 0 & 3 & 10 & 0 & 0 & 0 & 13 \\
\hline & 0 & 6 & 8 & 0 & 11 & 0 & 25 \\
\hline 7 & 0 & 5 & $\mathrm{~L}$ & $\mathrm{~L}$ & $\mathrm{~L}$ & $\mathrm{~L}$ & $\mathrm{~L}$ \\
\hline 8 & 0 & 0 & 5 & 10 & 0 & 1 & 16 \\
\hline 9 & 0 & 0 & 6 & 10 & 0 & 6 & 22 \\
\hline 10 & 0 & 4 & 7 & 0 & 12 & 0 & 23 \\
\hline
\end{tabular}

\begin{tabular}{|c|c|c|c|c|c|c|c|}
\hline \multicolumn{3}{|c|}{ Reproduction at } & $6.0 \%$ & \multicolumn{1}{c|}{ Concentration } \\
\hline Rep. & Day 2/3 & Day 4 & Day 5 & Day 6 & Day 7 & Day 8 & Total \\
\hline 1 & 0 & 2 & 0 & L & L & L & L \\
\hline 2 & 0 & 4 & 0 & 0 & 0 & 0 & 4 \\
\hline 3 & 0 & 3 & 4 & 0 & 5 & 6 & 18 \\
\hline 4 & 0 & 0 & 7 & 13 & 0 & 0 & 20 \\
\hline 5 & 0 & 4 & 4 & 0 & 0 & L & L \\
\hline 6 & 0 & 2 & 5 & 0 & 7 & 9 & 23 \\
\hline 7 & 0 & 5 & 8 & 0 & 6 & 0 & 19 \\
\hline 8 & 0 & 5 & 5 & 0 & 0 & 6 & 16 \\
\hline 9 & 0 & 5 & 8 & 13 & 0 & 0 & 26 \\
\hline 10 & 0 & 0 & 6 & 0 & 0 & 0 & 6 \\
\hline
\end{tabular}

\begin{tabular}{|c|c|c|c|c|c|c|c|}
\hline \multicolumn{1}{|c|}{ Reproduction at } & $12.0 \%$ & \multicolumn{1}{l|}{ Concentration } \\
\hline Rep. & Day 2/3 & Day 4 & Day 5 & Day 6 & Day 7 & Day 8 & Total \\
\hline 1 & 0 & 3 & 7 & 0 & 6 & 0 & 16 \\
\hline 2 & 0 & 5 & 7 & 0 & 14 & 0 & 26 \\
\hline 3 & 0 & 0 & 0 & 0 & 0 & L & L \\
\hline 4 & 0 & 4 & 8 & 0 & 5 & 0 & 17 \\
\hline 5 & 0 & 5 & 7 & 0 & 10 & 0 & 22 \\
\hline 6 & 0 & 0 & 0 & 0 & 8 & 10 & 18 \\
\hline 7 & 0 & $L$ & 0 & 0 & 0 & L & L \\
\hline 8 & 0 & 0 & 7 & 0 & 0 & 8 & 15 \\
\hline 9 & 0 & 4 & 8 & 0 & 0 & 0 & 12 \\
\hline 10 & 0 & 0 & 0 & 0 & 7 & 5 & 12 \\
\hline
\end{tabular}

\begin{tabular}{|c|c|c|c|c|c|c|c|}
\hline \multicolumn{3}{|c|}{ Reproduction at } & $25.0 \%$ & \multicolumn{5}{c|}{ Concentration } \\
\hline Rep. & Day 2/3 & Day 4 & Day 5 & Day 6 & Day 7 & Day 8 & Total \\
\hline 1 & 0 & 0 & 8 & 0 & 9 & 0 & 17 \\
\hline 2 & 0 & 3 & 8 & 0 & 4 & $\mathrm{~L}$ & 15 \\
\hline 3 & 0 & 0 & 5 & 10 & 0 & 11 & 26 \\
\hline 4 & 0 & 2 & 10 & 4 & 0 & 7 & 23 \\
\hline 5 & 0 & 4 & $L$ & 0 & 0 & 0 & $\mathrm{~L}$ \\
\hline 6 & 0 & 6 & $\mathrm{~L}$ & 0 & 8 & 10 & 24 \\
\hline 7 & 0 & 5 & $\mathrm{~L}$ & 0 & 0 & 0 & $\mathrm{~L}$ \\
\hline 8 & 0 & 1 & 5 & 0 & 0 & 8 & 14 \\
\hline 9 & 0 & 6 & 8 & 6 & 0 & 0 & 20 \\
\hline 10 & 0 & 4 & 1 & 0 & 11 & 0 & 16 \\
\hline
\end{tabular}

\begin{tabular}{|c|c|c|c|c|c|c|c|}
\hline \multicolumn{3}{|c|}{ Reproduction at } & \multirow{2}{*}{$\frac{50.0 \%}{\text { Day } 5}$} & \multicolumn{4}{|c|}{ Concentration } \\
\hline Rep. & Day $2 / 3$ & Day 4 & & Day 6 & Day 7 & Day 8 & Total \\
\hline 1 & 0 & 4 & 8 & 0 & $L$ & $\mathrm{~L}$ & $\mathrm{~L}$ \\
\hline 2 & 0 & 4 & 0 & $\overline{0}$ & 3 & 4 & 11 \\
\hline 3 & 0 & 4 & 2 & 0 & $\bar{X}$ & 0 & 6 \\
\hline 4 & 0 & 1 & 0 & 0 & 0 & $L$ & $\mathrm{~L}$ \\
\hline 5 & 0 & 4 & 1 & 0 & 10 & 0 & 15 \\
\hline 6 & 0 & 7 & 3 & 0 & 7 & 0 & 17 \\
\hline 7 & 0 & 0 & 0 & $\overline{0}$ & 9 & 15 & 24 \\
\hline 8 & 0 & 4 & 1 & 0 & 0 & 0 & 5 \\
\hline 9 & 0 & 0 & $L$ & $L$ & L & L & L \\
\hline 10 & 0 & 4 & 3 & 0 & 5 & 14 & 26 \\
\hline
\end{tabular}

\begin{tabular}{|c|c|c|c|c|c|c|c|}
\hline \multicolumn{3}{|c|}{ Reproduction at } & \multicolumn{5}{|c|}{$100.0 \%$ Concentration } \\
\hline Rep. & Day 2/3 & Day 4 & Day 5 & Day 6 & Day 7 & Day 8 & Total \\
\hline 1 & 0 & 4 & 4 & 0 & 8 & 0 & 16 \\
\hline 2 & 0 & $X$ & $\mathrm{X}$ & $x$ & $x$ & $x$ & 0 \\
\hline 3 & 0 & 5 & 7 & 15 & 0 & 0 & 27 \\
\hline 4 & 0 & 4 & 4 & 0 & 10 & 0 & 18 \\
\hline 5 & 0 & 3 & 0 & 0 & 10 & 10 & 23 \\
\hline 6 & 0 & 5 & 9 & 0 & 4 & 0 & 18 \\
\hline 7 & 0 & 1 & $\overline{0}$ & 0 & 9 & 10 & 20 \\
\hline 8 & 0 & $\bar{x}$ & $\bar{X}$ & $\bar{X}$ & $\bar{x}$ & $\bar{X}$ & 0 \\
\hline 9 & $\overline{0}$ & 5 & 6 & 13 & 0 & 0 & 24 \\
\hline 10 & 0 & 2 & 7 & 0 & 8 & 0 & 17 \\
\hline
\end{tabular}

$X=$ Dead

$L=$ Lost or caught in surface tension of water 


\section{CHRONIC DEFINITIVE SURVIVAL AND REPRODUCTION TEST \\ Statistical Analyses}

Client: Westinghouse Savannah River Company

Sample Identification: T6097 (A-001A)

Test Date: $\quad$ 7-16-96

\begin{tabular}{|ccc|}
\hline Test for Normality & & \\
Test Used: Shapiro Wilks & $\mathrm{W}=$ & 0.970 \\
& critical & 0.93 \\
The data are normal in distribution & \\
& \\
\end{tabular}

\begin{tabular}{|c|c|c|}
\hline \multicolumn{3}{|c|}{ Test for Homogeneity of Variance } \\
\hline Test Used: Bartlett's Test & $\begin{array}{r}\mathrm{B}= \\
\text { critical }\end{array}$ & $\begin{array}{l}7.04 \\
15.1\end{array}$ \\
\hline \multicolumn{3}{|c|}{ The data are homogeneous in variance } \\
\hline
\end{tabular}

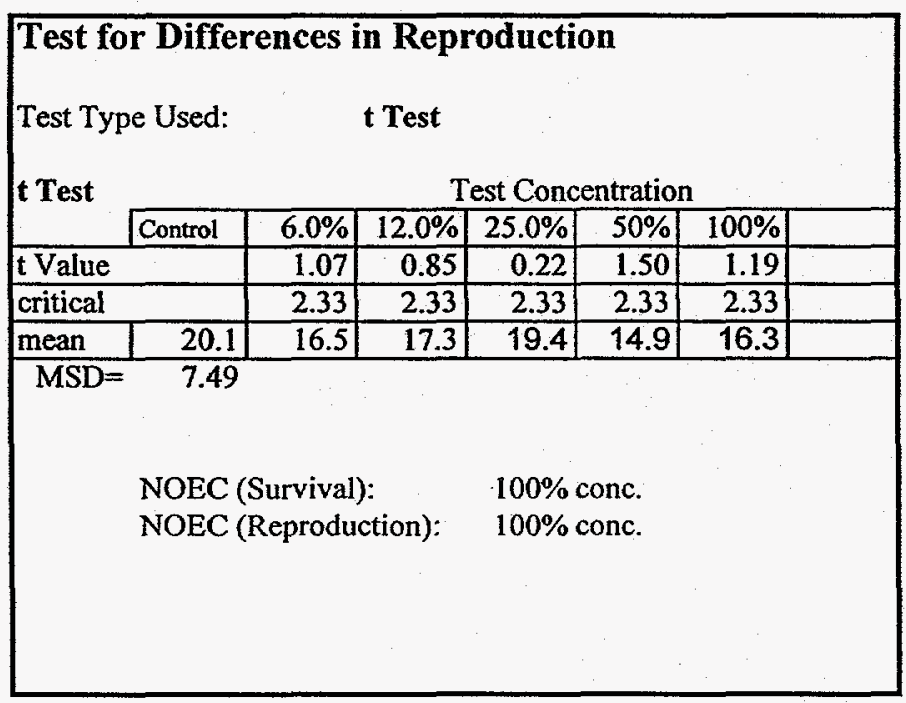

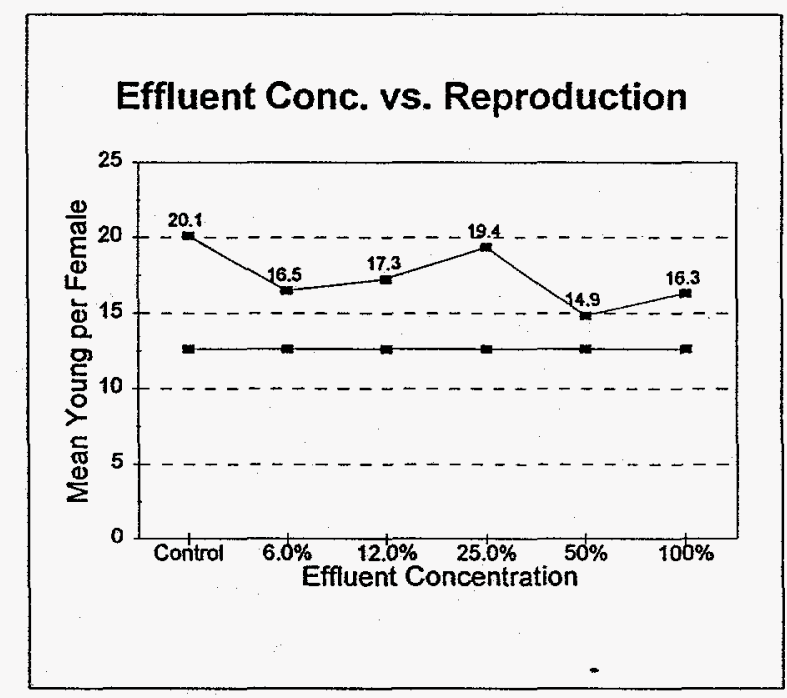

Summary

The effluent is not toxic. 


\section{CHRONIC DEFINITIVE SURVIVAL AND REPRODUCTION TEST \\ Water Chemistry}

Client: Westinghouse Savannah River Company

Sample Identification: T6097 (A-001A)

Test Date: $\quad$ 7-16-96

\section{pH (After Renewal)}

\begin{tabular}{|c|c|c|c|c|c|c|c|}
\hline & \multicolumn{7}{|c|}{ Test Concentration } \\
\hline & Control & $6.0 \%$ & $12.0 \%$ & $25.0 \%$ & $50 \%$ & $100 \%$ & \\
\hline Initial & 7.97 & 7.50 & 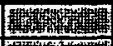 & 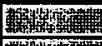 & 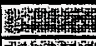 & 7.07 & \\
\hline Day 1 & 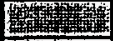 & 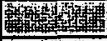 & - & 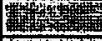 & 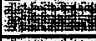 & 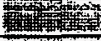 & Haty \\
\hline Day 2 & 9 & By & 10 & 4 & Whind & 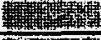 & 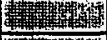 \\
\hline Day 3 & (1) & 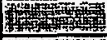 & 型 & (t) & Th & He & 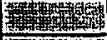 \\
\hline Day 4 & 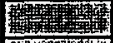 & 怔 & 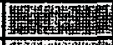 & 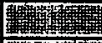 & 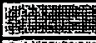 & 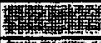 & DIn \\
\hline Day 5 & 111.1. & 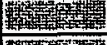 & 10 & 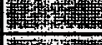 & 194 & $4+1$ & 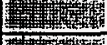 \\
\hline Day 6 & 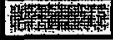 & 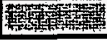 & 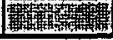 & 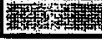 & 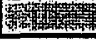 & 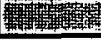 & 型) \\
\hline
\end{tabular}

Dissolved Oxygen (After Renewal)

\begin{tabular}{|c|c|c|c|c|c|c|c|}
\hline & \multicolumn{7}{|c|}{ Test Concentration } \\
\hline & Control & $6.0 \%$ & $12.0 \%$ & $25.0 \%$ & $50 \%$ & $100 \%$ & \\
\hline Initial & 8.6 & 8.6 & 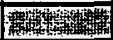 & 4 & 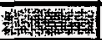 & 8.8 & \\
\hline Day 1 & 8.5 & & 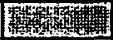 & 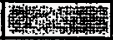 & 望 & & \\
\hline Day 2 & 8.6 & & 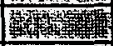 & 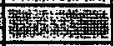 & 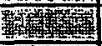 & & \\
\hline Day 3 & 8.7 & & 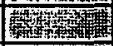 & Hen & 194 & & \\
\hline Day 4 & 8.6 & & 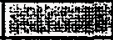 & 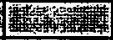 & Ty & & \\
\hline Day 5 & 8.6 & & 74 & 4 & 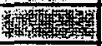 & & \\
\hline Day 6 & 8.7 & & 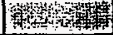 & | & 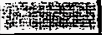 & & \\
\hline
\end{tabular}

Dilution Water \& Effluent Parameters

\begin{tabular}{|c|c|c|c|c|c|c|}
\hline & Res $\mathrm{Cl}$. & Alkalin & Hard. & Cond. & 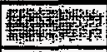 & 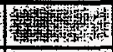 \\
\hline & $\mathrm{mg} / \mathrm{L}$ & $\mathrm{mg} / \mathrm{L}$ & $\mathrm{mg} / \mathrm{L}$ & $\mu \mathrm{mhos}$ & 19 & 17 \\
\hline Eff. Sample 1 & 0.17 & 2.1 & 6.0 & 32.9 & 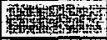 & 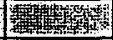 \\
\hline Eff. Sample 2 & 0.04 & 2.1 & 6.0 & 33.8 & 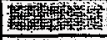 & 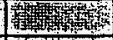 \\
\hline \multirow[t]{2}{*}{ Eff. Sample 3} & $\mathrm{~N} / \mathrm{A}$ & 2.1 & 10.0 & 33.8 & 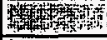 & The \\
\hline & & & & & Hin & 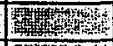 \\
\hline \multirow[t]{3}{*}{ Dilution Water } & & 2.1 & 4.0 & 30 & (4) & 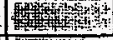 \\
\hline & & & & & Hotsty & 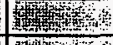 \\
\hline & & & & & 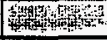 & 40 \\
\hline
\end{tabular}

$\mathrm{pH}$ (Before Renewal)

\begin{tabular}{|c|c|c|c|c|c|c|}
\hline & \multicolumn{6}{|c|}{ Test Concentration } \\
\hline & Control & $6.0 \%$ & $12.0 \%$ & $25.0 \%$ & $50 \%$ & $100 \%$ \\
\hline Day 1 & 8.18 & 7.24 & (1) & t & 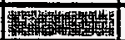 & 7.22 \\
\hline Day 2 & 8.09 & 7.83 & 倕 & 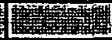 & 4 & 7.53 \\
\hline Day 3 & 8.24 & 7.75 & (1) & $\sqrt{14}$ & 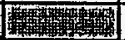 & 7.36 \\
\hline Day 4 & 8.19 & 7.34 & 14 & 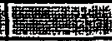 & 14 & 7.05 \\
\hline Day 5 & 7.98 & 7.34 & (1) & 9 & (7) & 7.05 \\
\hline Day 6 & 8.17 & 8.15 & 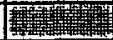 & 4 & 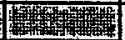 & 7.51 \\
\hline Final & 8.19 & 7.55 & 1) & 9 & 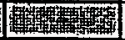 & 7.12 \\
\hline
\end{tabular}

Dissolved Oxygen (Before Renewal)

\begin{tabular}{|c|c|c|c|c|c|c|c|}
\hline & \multicolumn{7}{|c|}{ Test Concentration } \\
\hline & Control & $6.0 \%$ & $12.0 \%$ & $25.0 \%$ & $50 \%$ & $100 \%$ & \\
\hline Day 1 & 8.2 & 8.4 & 标 & 12 & $=1$ & 8.3 & \\
\hline Day 2 & 8.5 & 8.4 & (1) & - & 3 & 8.5 & \\
\hline Day 3 & 8.5 & 8.3 & II & 4 & 5 & 8.2 & \\
\hline Day 4 & 8.6 & 8.1 & 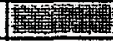 & $=1$ & (n) & 8.2 & \\
\hline Day 5 & 8.5 & 7.9 & 14 & 1 & +1 & 8.2 & \\
\hline Day 6 & 8.6 & 7.9 & (1) & He & 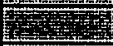 & 8.0 & \\
\hline Final. & 8.6 & 8.3 & 集 & Why & 14 & 8.3 & \\
\hline
\end{tabular}

\section{Temperature}

\begin{tabular}{|l|r|}
\hline \multicolumn{2}{|c|}{ Incubator ${ }^{\circ} \mathrm{C}$} \\
\hline Initial & 24.2 \\
\hline Day 1 & 24.3 \\
\hline Day 2 & 24.3 \\
\hline Day 3 & 24.4 \\
\hline Day 4 & 25.1 \\
\hline Day 5 & 25.9 \\
\hline Day 6 & 24.5 \\
\hline Final & 24.4 \\
\hline
\end{tabular}

Dilution Water was Upper Three Runs Creek Water pH Adjusted to 6.5 


\title{
7 Day Chronic Definitive Survival and Reproduction Bioassay
}

Method: EPA/600/4-89/001

\author{
Test Organism: Ceriodaphnia dubia
}

Facility:Westinghouse Savannah River Company

Sample ID:Outfall A-001A

Date: $\quad 8 / 06 / 96$ 


\section{CHRONIC DEFINITIVE SURVIVAL AND REPRODUCTION TEST Survival and Reproduction Results}

Client: Westinghouse Savannah River Company

Sample Identification: Outfall A-001A

Test Date: $\quad$ 8/06/96 $\quad$ T6220

\begin{tabular}{|c|c|c|c|c|c|c|}
\hline \multicolumn{3}{|c|}{ Reproduction at } & $0.0 \%$ & \multicolumn{3}{c|}{ Concentration } \\
\hline Rep. & Day 2/3 & Day 4 & Day 5 & Day 6 & Day 7 & Total \\
\hline 1 & 0 & 4 & 9 & 10 & 0 & 23 \\
\hline 2 & 0 & 4 & 8 & 0 & 15 & 27 \\
\hline 3 & 3 & 6 & 9 & 0 & 0 & 18 \\
\hline 4 & 4 & 3 & 8 & 13 & 0 & 28 \\
\hline 5 & 0 & 4 & 10 & 9 & 0 & 23 \\
\hline 6 & 0 & 3 & 9 & 0 & 12 & 24 \\
\hline 7 & 0 & 4 & 4 & 0 & 10 & 18 \\
\hline 8 & 0 & 4 & 10 & 14 & 0 & 28 \\
\hline 9 & 4 & 4 & 9 & 0 & 0 & 17 \\
\hline 10 & 0 & 7 & 8 & 0 & 11 & 26 \\
\hline
\end{tabular}

\begin{tabular}{|c|c|c|c|c|c|c|}
\hline \multicolumn{3}{|c|}{ Reproduction at } & $6.25 \%$ & \multicolumn{3}{c|}{ Concentration } \\
\hline Rep. & Day 2/3 & Day 4 & Day 5 & Day 6 & Day 7 & Total \\
\hline 1 & 0 & 4 & 4 & 13 & 0 & 21 \\
\hline 2 & 0 & 5 & 1 & 14 & 0 & 20 \\
\hline 3 & 4 & 0 & 8 & 12 & 0 & 24 \\
\hline 4 & 4 & 0 & 6 & 10 & 0 & 20 \\
\hline 5 & 0 & 0 & 4 & 0 & 11 & 15 \\
\hline 6 & 0 & 0 & 6 & 0 & 10 & 16 \\
\hline 7 & 0 & 3 & 11 & 10 & 0 & 24 \\
\hline 8 & 0 & 4 & 7 & 13 & 0 & 24 \\
\hline 9 & 4 & 0 & 8 & 14 & 0 & 26 \\
\hline 10 & 0 & 4 & 7 & 12 & 0 & 23 \\
\hline
\end{tabular}

\begin{tabular}{|c|c|c|c|c|c|c|}
\hline \multicolumn{3}{|c|}{ Reproduction at } & $12.5 \%$ & \multicolumn{2}{|c|}{ Concentration } & \multirow[b]{2}{*}{ Total } \\
\hline Rep. & Day $2 / 3$ & Day 4 & Day 5 & $\overline{\text { Day } 6}$ & $\overline{\text { Day } 7}$ & \\
\hline 1 & 0 & 3 & 8 & 14 & 0 & 25 \\
\hline 2 & 0 & 1 & 7 & 9 & 0 & 17 \\
\hline 3 & 3 & $\overline{0}$ & 8 & 13 & $\overline{0}$ & 24 \\
\hline$\overline{4}$ & 4 & 0 & $\overline{9}$ & 15 & 0 & 28 \\
\hline 5 & 0 & 4 & 9 & 3 & 12 & 28 \\
\hline 6 & 0 & 4 & 6 & 0 & 16 & 26 \\
\hline 7 & 0 & 0 & 10 & 5 & 8 & 23 \\
\hline 8 & 0 & 0 & 10 & 14 & 0 & 24 \\
\hline 9 & 4 & 0 & 8 & 14 & 0 & 26 \\
\hline 10 & 0 & 4 & 7 & 0 & 8 & 19 \\
\hline
\end{tabular}

\begin{tabular}{|c|c|c|c|c|c|c|}
\hline \multicolumn{3}{|c|}{ Reproduction at } \\
\hline Rep. & Day 2/3 & Day 4 & Day 5 & Day 6 & Day 7 & Total \\
\hline 1 & 0 & 4 & 7 & 0 & 11 & 22 \\
\hline 2 & 0 & 4 & 1 & 12 & 0 & 17 \\
\hline 3 & 2 & 0 & 8 & 12 & 0 & 22 \\
\hline 4 & 4 & 0 & 12 & 13 & 0 & 29 \\
\hline 5 & 0 & 0 & 8 & 0 & 12 & 20 \\
\hline 6 & 0 & 0 & 4 & 9 & 0 & 13 \\
\hline 7 & 0 & 0 & 7 & 0 & 7 & 14 \\
\hline 8 & 0 & 4 & 8 & 8 & 0 & 20 \\
\hline 9 & 4 & 0 & 4 & 14 & 0 & 22 \\
\hline 10 & 0 & 4 & 10 & 11 & 0 & 25 \\
\hline
\end{tabular}

\begin{tabular}{|c|c|c|c|c|c|c|}
\hline \multicolumn{3}{|c|}{ Reproduction at } & $50.0 \%$ & \multicolumn{3}{c|}{ Concentration } \\
\hline Rep. & Day $2 / 3$ & Day 4 & Day 5 & Day 6 & Day 7 & Total \\
\hline 1 & 0 & 0 & 6 & 0 & 9 & 15 \\
\hline 2 & 0 & 0 & 6 & 13 & 0 & 19 \\
\hline 3 & 4 & 0 & 9 & 15 & 0 & 28 \\
\hline 4 & 4 & 0 & 6 & 12 & 0 & 22 \\
\hline 5 & 0 & 3 & 13 & 16 & 0 & 32 \\
\hline 6 & 0 & 0 & 7 & 0 & 15 & 22 \\
\hline 7 & 0 & 0 & 11 & 4 & 0 & 15 \\
\hline 8 & 0 & 1 & 4 & 11 & 0 & 16 \\
\hline 9 & 4 & 0 & 4 & 14 & 0 & 22 \\
\hline 10 & 0 & 0 & 5 & 13 & 0 & 18 \\
\hline
\end{tabular}

\begin{tabular}{|c|c|c|c|c|c|c|}
\hline \multicolumn{3}{|c|}{ Reproduction at } & \multicolumn{4}{|c|}{$100.0 \%$ Concentration } \\
\hline Rep. & Day $2 / 3$ & Day 4 & Day 5 & Day 6 & Day 7 & Total \\
\hline 1 & 0 & 3 & 7 & 17 & 0 & 27 \\
\hline 2 & $\mathrm{~L}$ & $\mathrm{~L}$ & $\mathrm{~L}$ & $\mathrm{~L}$ & L & $\bar{L}$ \\
\hline 3 & 4 & 0 & 8 & 6 & 0 & 18 \\
\hline 4 & 0 & 4 & 4 & 6 & 1 & 15 \\
\hline 5 & $\begin{array}{l}0 \\
\end{array}$ & 4 & 9 & 14 & 0 & 27 \\
\hline 6 & 4 & $\begin{array}{l}0 \\
\end{array}$ & 6 & 0 & 11 & 21 \\
\hline 7 & 0 & 4 & 9 & $\overline{0}$ & 13 & 26 \\
\hline 8 & $\overline{0}$ & 4 & 11 & $\overline{12}$ & 0 & 27 \\
\hline 9 & $\bar{L}$ & $\bar{L}$ & $\mathrm{~L}$ & $\mathrm{~L}$ & $\bar{L}$ & $\mathrm{~L}$ \\
\hline 10 & 0 & 4 & 7 & 0 & 14 & 25 \\
\hline
\end{tabular}

$X=$ Dead

$L=$ Lost or caught in surface tension of water 


\section{CHRONIC DEFINITIVE SURVIVAL AND REPRODUCTION TEST \\ Statistical Analyses}

Client: Westinghouse Savannah River Company

Sample Identification: Outfall A-001A

Test Date: $\quad$ 8/06/96

\section{Test for Normality}

Test Used: Shapiro Wilks

$$
\begin{array}{rr}
\mathrm{W}= & 0.944 \\
\text { critical } & 0.93
\end{array}
$$

The data are normal in distribution
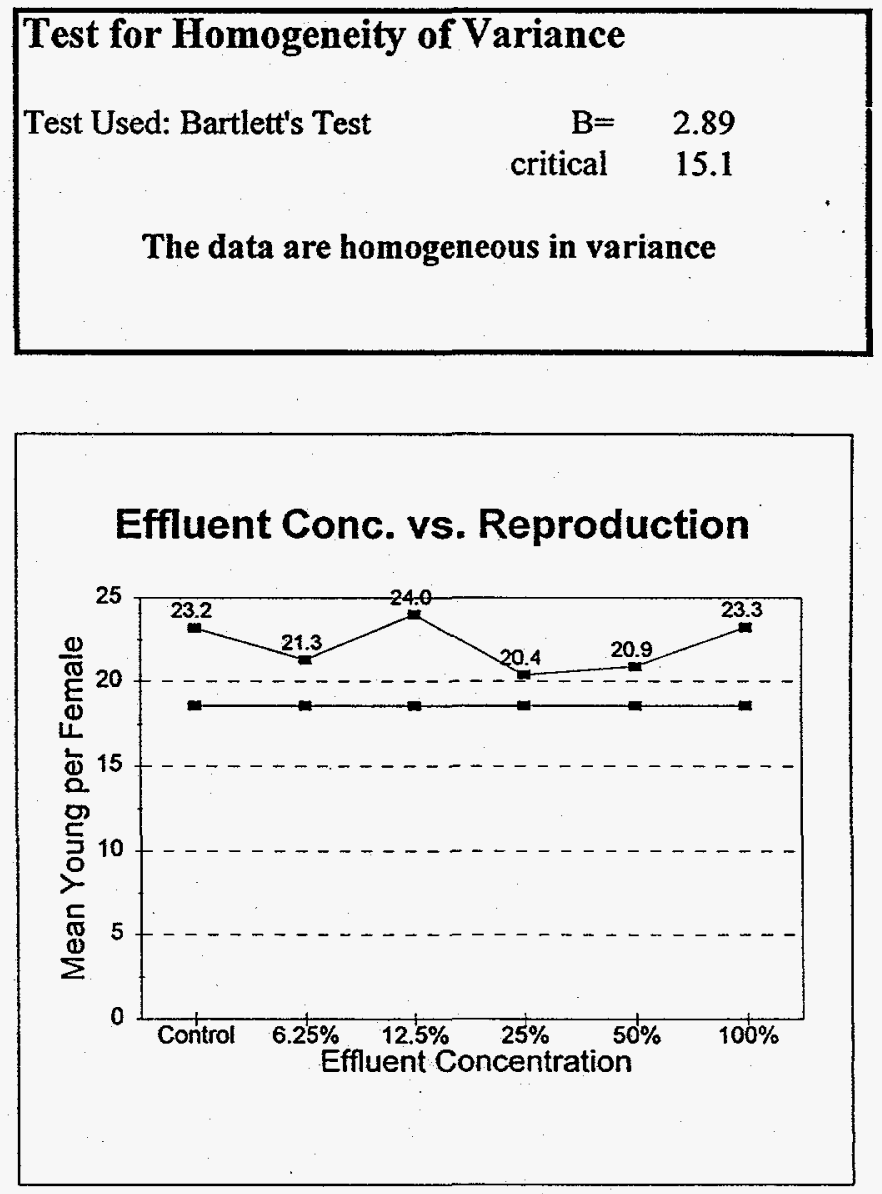

Summary

The effluent is not chronically toxic. 


\section{CHRONIC DEFINITIVE SURVIVAL AND REPRODUCTION TEST \\ Water Chemistry}

Client: Westinghouse Savannah River Company

Sample Identification: Outfall A-001A

Test Date: $\quad 8 / 06 / 96$

pH (After Renewal)

\begin{tabular}{|c|c|c|c|c|c|c|}
\hline & \multicolumn{6}{|c|}{ Test Concentration } \\
\hline & Control & $6.25 \%$ & $12.5 \%$ & $25 \%$ & $50 \%$ & $100 \%$ \\
\hline Initial & 6.50 & 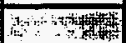 & 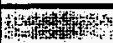 & 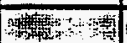 & W & 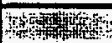 \\
\hline Day 1 & 6.50 & 92444 & 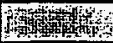 & ang & 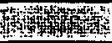 & 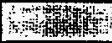 \\
\hline Day 2 & 6.50 & 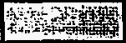 & (1) & 19 & $\mathrm{H}^{2}$ & Ifin \\
\hline Day 3 & 6.50 & 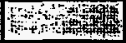 & 10 & rith & 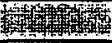 & 19te \\
\hline$\overline{\text { Day } 4}$ & 6.50 & 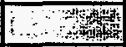 & P. & Dh & 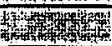 & 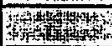 \\
\hline Day 5 & 6.50 & rex & 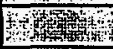 & $V^{2}$ & 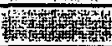 & 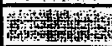 \\
\hline Day 6 & 6.50 & 3thts & 11t? & 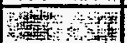 & (1) & 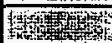 \\
\hline
\end{tabular}

Dissolved Oxygen (After Renewal)

\begin{tabular}{|c|c|c|c|c|c|c|}
\hline & \multicolumn{6}{|c|}{ Test Concentration } \\
\hline & Control & $6.25 \%$ & $12.5 \%$ & $25 \%$ & $50 \%$ & $100 \%$ \\
\hline Initial & 8.7 & 3 & Det & 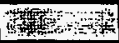 & 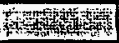 & 19 \\
\hline Day 1 & 8.7 & 17. & 12 & 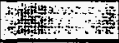 & Th & 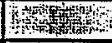 \\
\hline Day 2 & 8.7 & 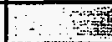 & ${ }^{2}$ & 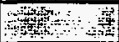 & 3) & 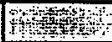 \\
\hline Day 3 & 8.7 & 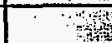 & Patyos & , & 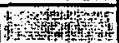 & .utw \\
\hline Day 4 & 8.7 & 3 & intwits & In & Bant & DT \\
\hline Day 5 & 8.7 & בip & 1) & : & Saty & Fing \\
\hline Day 6 & 8.7 & 78 & 3y & F. & $t^{2}$ & 5 \\
\hline
\end{tabular}

Dilution Water \& Effluent Parameters

\begin{tabular}{|c|c|c|c|c|c|}
\hline & Res Cl. & Alkalin & Hard. & Cond. & m. \\
\hline & $\mathrm{mg} / \mathrm{L}$ & $\mathrm{mg} / \mathrm{L}$ & $\mathrm{mg} / \mathrm{L}$ & $\mu$ mhos & trat \\
\hline Eff. Sample 1 & 0.07 & 4.2 & 4 & 33.9 & the \\
\hline Eff. Sample 2 & 0.06 & 2.1 & 10 & 79.2 & 7 \\
\hline \multirow[t]{2}{*}{ Eff. Sample 3} & N/A & 2.1 & 6 & 33.2 & sits \\
\hline & & & & & 8 \\
\hline \multirow[t]{3}{*}{ Dilution Water } & & 4 & 4 & 15 & 8 \\
\hline & & & & & i!: \\
\hline & & & & & I \\
\hline
\end{tabular}

$\mathrm{pH}$ (Before Renewal)

\begin{tabular}{|c|c|c|c|c|c|c|}
\hline & \multicolumn{6}{|c|}{ Test Concentration } \\
\hline & Control & $6.25 \%$ & $12.5 \%$ & $25 \%$ & $50 \%$ & $100 \%$ \\
\hline Day 1 & 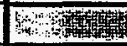 & 8.04 & 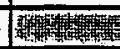 & F & Thi & \\
\hline Day 2 & Bnty & $\overline{7.40}$ & 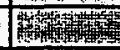 & Find & Henty & 6.23 \\
\hline Day 3 & 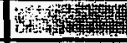 & 7.76 & 12 & (1) & 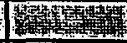 & 7.68 \\
\hline Day 4 & 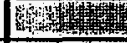 & 7.70 & |10 & (3) & $y^{2}$ & 7.71 \\
\hline Day 5 & $H_{3}$ & 7.57 & 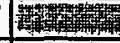 & $\sqrt{50}$ & 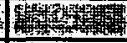 & 8.03 \\
\hline Day 6 & Fide & 7.05 & 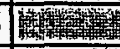 & 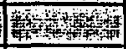 & W & 6.91 \\
\hline Final & Inturat & 6.65 & $\mid$ & 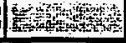 & 10 & 6.62 \\
\hline
\end{tabular}

Dissolved Oxygen (Before Renewal)

\begin{tabular}{|c|c|c|c|c|c|c|}
\hline & \multicolumn{6}{|c|}{ Test Concentration } \\
\hline & Control & $6.25 \%$ & $12.5 \%$ & $25 \%$ & $50 \%$ & $100 \%$ \\
\hline Day 1 & 8.4 & 8.6 & 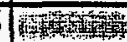 & $x^{2}+3$ & 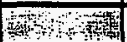 & \\
\hline Day 2 & 8.5 & 8.2 & 1) & Spln & 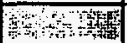 & 8.3 \\
\hline Day 3 & 8.4 & 8.5 & 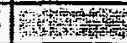 & mas & मn: & 8.5 \\
\hline Day 4 & 8.3 & $\overline{8.4}$ & 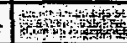 & WAs & ma & 8.4 \\
\hline Day 5 & 8.7 & $\overline{8.4}$ & 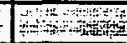 & Bas & and & 8.5 \\
\hline Day 6 & 8.8 & 8.6 & WP & Ea & & 8.7 \\
\hline Final & 8.7 & 8.7 & rivity & 5 & ... & 8.7 \\
\hline
\end{tabular}

Temperature

\begin{tabular}{|l|r|}
\hline \multicolumn{2}{|c|}{ Incubator ${ }^{\circ} \mathrm{C}$} \\
\hline Initial & 26.0 \\
\hline Day 1 & 24.3 \\
\hline Day 2 & 24.6 \\
\hline Day 3 & 24.6 \\
\hline Day 4 & 26.0 \\
\hline Day 5 & 25.0 \\
\hline Day 6 & 25.0 \\
\hline Final & 25.2 \\
\hline
\end{tabular}

Dilution water $\mathrm{pH}$

adjusted daily to $\mathrm{pH} 6.5$ 


\title{
7 Day Chronic Definitive Survival and Reproduction Bioassay \\ Method: EPA/600/4-89/001
}

\author{
Test Organism: Ceriodaphnia dubia
}

Facility: Westinghouse Savannah River Company

Sample ID: A-001C

Date: $\quad 6 / 25 / 96$ 


\section{CHRONIC DEFINITIVE SURVIVAL AND REPRODUCTION TEST Survival and Reproduction Results}

Client: Westinghouse Savannah River Company

Sample Identification: A-001C

Test Date:

$6 / 25 / 96$

\begin{tabular}{|c|c|c|c|c|c|c|}
\hline \multicolumn{3}{|c|}{ Reproduction at } & $0.0 \%$ & \multicolumn{3}{c|}{ Concentration } \\
\hline Rep. & Day 3/4 & Day 5 & Day 6 & Day 7 & Day 8 & Total \\
\hline 1 & 0 & 3 & 9 & 0 & 0 & 12 \\
\hline 2 & 1 & 2 & 0 & 10 & 10 & 23 \\
\hline 3 & 0 & 3 & 10 & 0 & 9 & 22 \\
\hline 4 & 3 & 6 & 7 & 0 & 11 & 27 \\
\hline 5 & 3 & 4 & 4 & 0 & 13 & 24 \\
\hline 6 & 0 & 6 & 0 & 15 & 0 & 21 \\
\hline 7 & 3 & 7 & 10 & 0 & 0 & 20 \\
\hline 8 & 0 & 4 & 0 & 5 & 0 & 9 \\
\hline 9 & 2 & 6 & 9 & 0 & 0 & 17 \\
\hline 10 & 3 & 0 & 0 & 2 & 13 & 18 \\
\hline
\end{tabular}

\begin{tabular}{|c|c|c|c|c|c|c|}
\hline \multicolumn{3}{|c|}{ Reproduction at } & $6.25 \%$ & \multicolumn{4}{c|}{ Concentration } \\
\hline Rep. & Day 3/4 & Day 5 & Day 6 & Day 7 & Day 8 & Total \\
\hline 1 & 0 & 0 & 1 & 5 & 8 & 14 \\
\hline 2 & 0 & 0 & 1 & 0 & 0 & 1 \\
\hline 3 & 0 & 0 & 0 & 0 & 4 & 4 \\
\hline 4 & 0 & 0 & 0 & 3 & 8 & 11 \\
\hline 5 & 0 & 0 & 0 & 0 & 1 & 1 \\
\hline 6 & 0 & 0 & 0 & 4 & 0 & 4 \\
\hline 7 & 0 & 0 & 1 & 2 & 11 & 14 \\
\hline 8 & 0 & 0 & 3 & 11 & 0 & 14 \\
\hline 9 & 0 & 0 & 0 & 0 & 13 & 13 \\
\hline 10 & 0 & 0 & 2 & 2 & 4 & 8 \\
\hline
\end{tabular}

\begin{tabular}{|c|c|c|c|c|c|c|}
\hline \multicolumn{3}{|c|}{ Reproduction at } & \multicolumn{1}{c|}{$12.5 \%$} & \multicolumn{3}{c|}{ Concentration } \\
\hline Rep. & Day 3/4 & Day 5 & Day 6 & Day 7 & Day 8 & Total \\
\hline 1 & 0 & 0 & 0 & $X$ & $X$ & 0 \\
\hline 2 & 0 & 3 & 0 & 8 & 5 & 16 \\
\hline 3 & 0 & 3 & 1 & 5 & 0 & 9 \\
\hline 4 & 0 & 0 & 0 & 12 & 0 & 12 \\
\hline 5 & 0 & 0 & 7 & 0 & 10 & 17 \\
\hline 6 & 0 & 0 & 0 & 13 & 0 & 13 \\
\hline 7 & 0 & 0 & 5 & 2 & 0 & 7 \\
\hline 8 & $X$ & $X$ & $X$ & $X$ & $X$ & 0 \\
\hline 9 & 0 & 0 & 0 & 14 & 0 & 14 \\
\hline 10 & 0 & 0 & 0 & 3 & 0 & 3 \\
\hline
\end{tabular}

\begin{tabular}{|c|c|c|c|c|c|c|}
\hline \multicolumn{3}{|c|}{ Reproduction at } & \multirow{2}{*}{$\frac{25.0 \%}{\text { Day } 6}$} & \multicolumn{2}{|c|}{ Concentration } & \multirow[b]{2}{*}{ Total } \\
\hline Rep. & Day $3 / 4$ & Day 5 & & Day 7 & Day 8 & \\
\hline 1 & $\bar{X}$ & $x$ & $\bar{x}$ & $x$ & $\bar{x}$ & 0 \\
\hline 2 & $\bar{x}$ & $\bar{x}$ & $\bar{x}$ & $\bar{x}$ & $\bar{x}$ & 0 \\
\hline 3 & $\bar{x}$ & $\sqrt{x}$ & $\bar{X}$ & $\bar{x}$ & $\mathrm{X}$ & 0 \\
\hline 4 & $\bar{X}$ & X & $\bar{X}$ & $\bar{x}$ & $\mathrm{X}$ & 0 \\
\hline 5 & $\bar{X}$ & $\bar{X}$ & $\bar{x}$ & X & $\bar{x}$ & 0 \\
\hline 6 & X & $\bar{x}$ & $\bar{x}$ & $\bar{x}$ & $\bar{x}$ & 0 \\
\hline 7 & $\bar{X}$ & $\bar{x}$ & $\bar{x}$ & $\bar{x}$ & $\bar{X}$ & 0 \\
\hline 8 & X & $\bar{x}$ & $\bar{x}$ & X & $\bar{x}$ & 0 \\
\hline 9 & X & $\mathrm{X}$ & $x$ & $x$ & X & 0 \\
\hline 10 & X. & $\mathrm{X}$ & $\bar{x}$ & X & $\mathrm{X}$ & 0 \\
\hline
\end{tabular}

\begin{tabular}{|c|l|l|l|l|l|l|}
\hline \multicolumn{2}{|l|}{ Reproduction at } & $50.0 \%$ & \multicolumn{3}{c|}{ Concentration } \\
\hline Rep. & Day 3/4 & Day 5 & Day 6 & Day 7 & Day 8 & Total \\
\hline 1 & $\mathrm{X}$ & $\mathrm{X}$ & $\mathrm{X}$ & $\mathrm{X}$ & $\mathrm{X}$ & 0 \\
\hline 2 & $\mathrm{X}$ & $\mathrm{X}$ & $\mathrm{X}$ & $\mathrm{X}$ & $\mathrm{X}$ & 0 \\
\hline 3 & $\mathrm{X}$ & $\mathrm{X}$ & $\mathrm{X}$ & $\mathrm{X}$ & $\mathrm{X}$ & 0 \\
\hline 4 & $\mathrm{X}$ & $\mathrm{X}$ & $\mathrm{X}$ & $\mathrm{X}$ & $\mathrm{X}$ & 0 \\
\hline 5 & $\mathrm{X}$ & $\mathrm{X}$ & $\mathrm{X}$ & $\mathrm{X}$ & $\mathrm{X}$ & 0 \\
\hline 6 & $\mathrm{X}$ & $\mathrm{X}$ & $\mathrm{X}$ & $\mathrm{X}$ & $\mathrm{X}$ & 0 \\
\hline 7 & $\mathrm{X}$ & $\mathrm{X}$ & $\mathrm{X}$ & $\mathrm{X}$ & $\mathrm{X}$ & 0 \\
\hline 8 & $\mathrm{X}$ & $\mathrm{X}$ & $\mathrm{X}$ & $\mathrm{X}$ & $\mathrm{X}$ & 0 \\
\hline 9 & $\mathrm{X}$ & $\mathrm{X}$ & $\mathrm{X}$ & $\mathrm{X}$ & $\mathrm{X}$ & 0 \\
\hline 10 & $\mathrm{X}$ & $\mathrm{X}$ & $\mathrm{X}$ & $\mathrm{X}$ & $\mathrm{X}$ & 0 \\
\hline
\end{tabular}

\begin{tabular}{|c|l|l|l|l|l|l|}
\hline \multicolumn{3}{|c|}{ Reproduction at } & \multicolumn{1}{c|}{$100 \%$} & \multicolumn{3}{c|}{ Concentration } \\
\hline Rep. & Day $3 / 4$ & Day 5 & Day 6 & Day 7 & Day 8 & Total \\
\hline I & $\mathrm{X}$ & $\mathrm{X}$ & $\mathrm{X}$ & $\mathrm{X}$ & $\mathrm{X}$ & 0 \\
\hline 2 & $\mathrm{X}$ & $\mathrm{X}$ & $\mathrm{X}$ & $\mathrm{X}$ & $\mathrm{X}$ & 0 \\
\hline 3 & $\mathrm{X}$ & $\mathrm{X}$ & $\mathrm{X}$ & $\mathrm{X}$ & $\mathrm{X}$ & 0 \\
\hline 4 & $\mathrm{X}$ & $\mathrm{X}$ & $\mathrm{X}$ & $\mathrm{X}$ & $\mathrm{X}$ & 0 \\
\hline 5 & $\mathrm{X}$ & $\mathrm{X}$ & $\mathrm{X}$ & $\mathrm{X}$ & $\mathrm{X}$ & 0 \\
\hline 6 & $\mathrm{X}$ & $\mathrm{X}$ & $\mathrm{X}$ & $\mathrm{X}$ & $\mathrm{X}$ & 0 \\
\hline 7 & $\mathrm{X}$ & $\mathrm{X}$ & $\mathrm{X}$ & $\mathrm{X}$ & $\mathrm{X}$ & 0 \\
\hline 8 & $\mathrm{X}$ & $\mathrm{X}$ & $\mathrm{X}$ & $\mathrm{X}$ & $\mathrm{X}$ & 0 \\
\hline 9 & $\mathrm{X}$ & $\mathrm{X}$ & $\mathrm{X}$ & $\mathrm{X}$ & $\mathrm{X}$ & 0 \\
\hline 10 & $\mathrm{X}$ & $\mathrm{X}$ & $\mathrm{X}$ & $\mathrm{X}$ & $\mathrm{X}$ & 0 \\
\hline
\end{tabular}

$X=$ Dead

$L=$ Lost or caught in surface tension of water 


\section{CHRONIC DEFINITIVE SURVIVAL AND REPRODUCTION TEST \\ Statistical Analyses}

Client: Westinghouse Savannah River Company

Sample Identification: A-001C

Test Date: $\quad 6 / 25 / 96$

\begin{tabular}{|crr|}
\hline Test for Normality & & \\
Test Used: Shapiro Wilks & $\mathrm{W}=$ & 0.886 \\
& critical & 0.93 \\
& & \\
The data are not normal in distribution & \\
\end{tabular}

\begin{tabular}{|crr|}
\hline Test for Homogeneity of Variance & \\
Test Used: Bartlett's Test & $\mathrm{B}=$ & 0.27 \\
& critical & 13.28 \\
& & \\
The data are homogeneous in variance
\end{tabular}

\begin{tabular}{|c|c|c|c|c|c|c|}
\hline \multicolumn{7}{|c|}{ Test for Differences in Reproduction } \\
\hline \multicolumn{2}{|c|}{ Test Type Used: } & \multicolumn{5}{|c|}{ Wilcoxon's Test } \\
\hline \multirow[t]{2}{*}{ t Test } & & \multicolumn{5}{|c|}{ Test Concentration } \\
\hline & Control & $6.25 \%$ & $12.5 \%$ & $25 \%$ & $50.0 \%$ & $100 \%$ \\
\hline \multicolumn{2}{|l|}{ t Value } & 5.43 & 5.08 & N/A & N/A & N/A \\
\hline \multicolumn{2}{|l|}{ critical } & 2.33 & 2.33 & N/A & N/A & N/A \\
\hline mean & 19.3 & 8.4 & 9.1 & 0.0 & 0.0 & 0.0 \\
\hline \multicolumn{7}{|c|}{$\mathrm{MSD}=4.68$} \\
\hline \multirow{2}{*}{\multicolumn{2}{|c|}{ Wilcoxon's Test }} & \multicolumn{5}{|c|}{ Test Concentration } \\
\hline & & $6.25 \%$ & $12.5 \%$ & $25 \%$ & $50.0 \%$ & $100 \%$ \\
\hline \multicolumn{2}{|c|}{ Rank Sum } & 61 & & & & \\
\hline \multirow[t]{2}{*}{ critical } & & 78 & & & & \\
\hline & $\begin{array}{l}\text { LOEC } \\
\text { NOEC }\end{array}$ & $6.25 \%$ & $\begin{array}{l}\text { effluent } \\
\text { effluent }\end{array}$ & & & \\
\hline
\end{tabular}

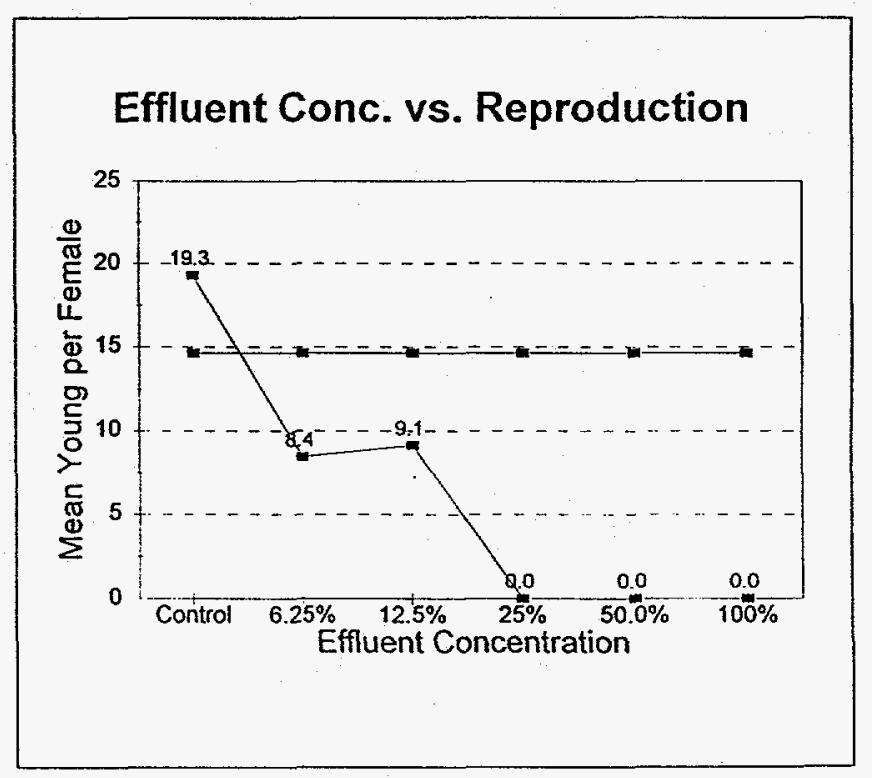

\section{Summary}

The effluent was toxic at all test concentrations (lowest concentration run was $6.25 \%$ ). 


\section{CHRONIC DEFINITIVE SURVIVAL AND REPRODUCTION TEST Water Chemistry}

Client: Westinghouse Savannah River Company

Sample Identification: A-001C

Test Date: $\quad 6 / 25 / 96$

pH (After Renewal)

\begin{tabular}{|l|r|r|r|r|r|r|}
\hline \multicolumn{7}{|c|}{ Test Concentration } \\
\hline & Control & $6.25 \%$ & $12.5 \%$ & $25 \%$ & $50.0 \%$ & $100 \%$ \\
\hline Initial & 8.28 & 7.89 & 7.67 & 7.63 & 7.59 & 7.56 \\
\hline Day 1 & 8.22 & 7.71 & 7.60 & & & \\
\hline Day 2 & 7.91 & 7.40 & 7.50 & & & \\
\hline Day 3 & 7.90 & 7.36 & 7.39 & & & \\
\hline Day 4 & 8.07 & 7.28 & 7.31 & & & \\
\hline Day 5 & 8.16 & 7.32 & 7.27 & & & \\
\hline Day 6 & 8.27 & 7.74 & 7.70 & & & \\
\hline
\end{tabular}

Dissolved Oxygen (After Renewal)

\begin{tabular}{|l|r|r|r|r|r|r|}
\hline \multicolumn{7}{|c|}{ Test Concentration } \\
\hline & Control & $6.25 \%$ & $12.5 \%$ & $25 \%$ & $50.0 \%$ & $100 \%$ \\
\hline Initial & 8.6 & 8.9 & 8.9 & 8.7 & 8.6 & 8.6 \\
\hline Day 1 & 8.7 & 8.8 & 8.9 & 8.9 & 8.6 & 8.6 \\
\hline Day 2 & 8.5 & 8.6 & 8.8 & & & \\
\hline Day 3 & 8.6 & 8.6 & 8.7 & & & \\
\hline Day 4 & 8.6 & 8.8 & 8.4 & & & \\
\hline Day 5 & 8.6 & 8.7 & 8.7 & & & \\
\hline Day 6 & 8.5 & 8.5 & 8.6 & & & \\
\hline
\end{tabular}

Dilution Water \& Effluent Parameters

\begin{tabular}{|l|r|r|r|r|l|}
\hline & Res Cl. & Alkalin & Hard. & Cond. & \\
\hline & $\mathrm{mg} / \mathrm{L}$ & $\mathrm{mg} / \mathrm{L}$ & $\mathrm{mg} / \mathrm{L}$ & $\mu$ mhos & \\
\hline Eff. Sample 1 & $<.01$ & 45.3 & 24 & 91.8 & \\
\hline Eff. Sample 2 & $<.01$ & 49.4 & 14 & 1072 & \\
\hline Eff. Sample 3 & 0.3 & 41.2 & 8 & 929 & \\
\hline & & & & & \\
\hline Dilution Water & & 70 & 84 & 187.9 & \\
\hline & & & & & \\
\hline & & & & & \\
\hline
\end{tabular}

$\mathrm{pH}$ (Before Renewal)

\begin{tabular}{|l|r|r|r|r|r|r|}
\hline \multicolumn{7}{|c|}{ Test Concentration } \\
\hline & Control & $6.25 \%$ & $12.5 \%$ & $25 \%$ & $50.0 \%$ & $100 \%$ \\
\hline Day 1 & 8.20 & 7.05 & 7.11 & 7.19 & 7.23 & 7.27 \\
\hline Day 2 & 8.17 & 7.56 & 7.84 & & & \\
\hline Day 3 & 8.36 & 7.84 & 7.93 & & & \\
\hline Day 4 & 8.22 & 8.23 & 7.99 & & & \\
\hline Day 5 & 8.20 & 7.39 & 7.45 & & & \\
\hline Day 6 & 8.24 & 7.77 & 7.73 & & & \\
\hline Final & 8.19 & 8.18 & 8.20 & & & \\
\hline
\end{tabular}

Dissolved Oxygen (Before Renewal)

\begin{tabular}{|l|r|r|r|r|r|r|}
\hline \multicolumn{7}{|c|}{ Test Concentration } \\
\hline & Control & $6.25 \%$ & $12.5 \%$ & $25 \%$ & $50.0 \%$ & $100 \%$ \\
\hline Day 1 & 8.6 & 8.7 & 8.7 & 8.6 & 8.5 & 8.3 \\
\hline Day 2 & 8.6 & 8.4 & 8.6 & & 8.5 & 8.3 \\
\hline Day 3 & 8.4 & 8.4 & 8.3 & & & \\
\hline Day 4 & 8.4 & 8.6 & 8.5 & & & \\
\hline Day 5 & 8.6 & 8.6 & 8.6 & & & \\
\hline Day 6 & 8.5 & 8.6 & 8.6 & & & \\
\hline Final & 8.5 & 8.0 & 8.0 & & & \\
\hline
\end{tabular}

\section{Temperature}

\begin{tabular}{|l|r|}
\hline \multicolumn{2}{|c|}{ Incubator ${ }^{\circ} \mathrm{C}$} \\
\hline Initial & 24.6 \\
\hline Day 1 & 24.6 \\
\hline Day 2 & 24.9 \\
\hline Day 3 & 24.1 \\
\hline Day 4 & 25.7 \\
\hline Day 5 & 24.7 \\
\hline Day 6 & 25.2 \\
\hline Final & 24.6 \\
\hline
\end{tabular}




\title{
7 Day Chronic Definitive Survival and Reproduction Bioassay \\ Method: EPA/600/4-89/001
}

\author{
Test Organism: Ceriodaphnia dubia
}

Facility: Westinghouse Savannah River Company

Sample ID: A-003 Effluent

Date: $\quad 6 / 25 / 96$ 


\section{CHRONIC DEFINITIVE SURVIVAL AND REPRODUCTION TEST Survival and Reproduction Results}

Client: Westinghouse Savannah River Company

Sample Identification: A-003

Test Date: $\quad$ 6/25/96

\begin{tabular}{|c|c|c|c|c|c|c|}
\hline \multicolumn{2}{|c|}{ Reproduction at } & \multicolumn{3}{c|}{$0 \%$} & \multicolumn{4}{|c|}{ Concentration } \\
\hline Rep. & Day 3/4 & Day 5 & Day 6 & Day 7 & Day 8 & Total \\
\hline 1 & 0 & 3 & 9 & 0 & 0 & 12 \\
\hline 2 & 1 & 2 & 0 & 10 & 10 & 23 \\
\hline 3 & 0 & 3 & 10 & 0 & 9 & 22 \\
\hline 4 & 3 & 6 & 7 & 0 & 11 & 27 \\
\hline 5 & 3 & 4 & 4 & 0 & 13 & 24 \\
\hline 6 & 0 & 6 & 0 & 15 & 0 & 21 \\
\hline 7 & 3 & 7 & 10 & 0 & 0 & 20 \\
\hline 8 & 0 & 4 & 0 & 5 & 0 & 9 \\
\hline 9 & 2 & 6 & 9 & 0 & 0 & 17 \\
\hline 10 & 3 & 0 & 0 & 2 & 13 & 18 \\
\hline
\end{tabular}

\begin{tabular}{|c|c|c|c|c|c|c|}
\hline \multicolumn{3}{|c|}{ Reproduction at } & \multicolumn{4}{|c|}{$12.5 \%$ Concentration } \\
\hline Rep. & Day 3/4 & Day 5 & Day 6 & Day 7 & Day 8 & Total \\
\hline 1 & 0 & 0 & 0 & 0 & 0 & 0 \\
\hline 2 & $\bar{X}$ & $\bar{X}$ & $\bar{x}$ & $\bar{X}$ & $\bar{X}$ & 0 \\
\hline 3 & $\bar{x}$ & $\bar{x}$ & $\bar{x}$ & $\bar{x}$ & $x$ & 0 \\
\hline 4 & $\bar{x}$ & $\bar{X}$ & $\bar{X}$ & $\bar{x}$ & $\bar{X}$ & 0 \\
\hline 5 & $\mathrm{X}$ & $x$ & $\bar{x}$ & $x$ & $\bar{x}$ & 0 \\
\hline 6 & $x$ & $\bar{X}$ & $\bar{x}$ & $\bar{x}$ & $\bar{x}$ & 0 \\
\hline 7 & $x$ & $\mathrm{X}$ & $\bar{X}$ & $\bar{x}$ & $\bar{x}$ & 0 \\
\hline 8 & $x$ & $\bar{x}$ & $x$ & $x$ & $\mathrm{X}$ & 0 \\
\hline 9 & $x$ & $x$ & $x$ & $\bar{X}$ & $x$ & 0 \\
\hline 10 & 0 & 0 & 0 & 0 & $\bar{X}$ & 0 \\
\hline
\end{tabular}

\begin{tabular}{|c|l|l|l|l|l|c|}
\hline \multicolumn{6}{|c|}{ Reproduction at } & \multicolumn{5}{l|}{$50.0 \%$} & \multicolumn{3}{l|}{ Concentration } \\
\hline Rep. & Day 3/4 & Day 5 & Day 6 & Day 7 & Day 8 & Total \\
\hline 1 & $\mathrm{X}$ & $\mathrm{X}$ & $\mathrm{X}$ & $\mathrm{X}$ & $\mathrm{X}$ & 0 \\
\hline 2 & $\mathrm{X}$ & $\mathrm{X}$ & $\mathrm{X}$ & $\mathrm{X}$ & $\mathrm{X}$ & 0 \\
\hline 3 & $\mathrm{X}$ & $\mathrm{X}$ & $\mathrm{X}$ & $\mathrm{X}$ & $\mathrm{X}$ & 0 \\
\hline 4 & $\mathrm{X}$ & $\mathrm{X}$ & $\mathrm{X}$ & $\mathrm{X}$ & $\mathrm{X}$ & 0 \\
\hline 5 & $\mathrm{X}$ & $\mathrm{X}$ & $\mathrm{X}$ & $\mathrm{X}$ & $\mathrm{X}$ & 0 \\
\hline 6 & $\mathrm{X}$ & $\mathrm{X}$ & $\mathrm{X}$ & $\mathrm{X}$ & $\mathrm{X}$ & 0 \\
\hline 7 & $\mathrm{X}$ & $\mathrm{X}$ & $\mathrm{X}$ & $\mathrm{X}$ & $\mathrm{X}$ & 0 \\
\hline 8 & $\mathrm{X}$ & $\mathrm{X}$ & $\mathrm{X}$ & $\mathrm{X}$ & $\mathrm{X}$ & 0 \\
\hline 9 & $\mathrm{X}$ & $\mathrm{X}$ & $\mathrm{X}$ & $\mathrm{X}$ & $\mathrm{X}$ & 0 \\
\hline 10 & $\mathrm{X}$ & $\mathrm{X}$ & $\mathrm{X}$ & $\mathrm{X}$ & $\mathrm{X}$ & 0 \\
\hline
\end{tabular}

$X=$ Dead

$L=$ Lost or caught in surface tension of water

\begin{tabular}{|c|c|c|c|c|c|c|}
\hline \multicolumn{3}{|c|}{ Reproduction at } & $6.2 \%$ & \multicolumn{3}{c|}{ Concentration } \\
\hline Rep. & Day 3/4 & Day 5 & Day 6 & Day 7 & Day 8 & Total \\
\hline 1 & $\mathrm{X}$ & $\mathrm{X}$ & $\mathrm{X}$ & $\mathrm{X}$ & $\mathrm{X}$ & 0 \\
\hline 2 & 0 & 1 & 2 & 0 & 5 & 8 \\
\hline 3 & $\mathrm{X}$ & $\mathrm{X}$ & $\mathrm{X}$ & $\mathrm{X}$ & $\mathrm{X}$ & 0 \\
\hline 4 & $\mathrm{X}$ & $\mathrm{X}$ & $\mathrm{X}$ & $\mathrm{X}$ & $\mathrm{X}$ & 0 \\
\hline 5 & $\mathrm{X}$ & $\mathrm{X}$ & $\mathrm{X}$ & $\mathrm{X}$ & $\mathrm{X}$ & 0 \\
\hline 6 & 0 & 3 & 5 & 0 & 14 & 22 \\
\hline 7 & 0 & 3 & 4 & 0 & 12 & 19 \\
\hline 8 & $\mathrm{X}$ & $\mathrm{X}$ & $\mathrm{X}$ & $\mathrm{X}$ & $\mathrm{X}$ & 0 \\
\hline 9 & 4 & 6 & 12 & 0 & 0 & 22 \\
\hline 10 & 3 & 0 & 0 & 9 & 7 & 19 \\
\hline
\end{tabular}

\begin{tabular}{|c|c|c|c|c|c|c|}
\hline \multicolumn{3}{|c|}{ Reproduction at } & \multicolumn{5}{c|}{ Concentration } \\
\hline Rep. & Day 3/4 & Day 5 & Day 6 & Day 7 & Day 8 & Total \\
\hline 1 & $\mathrm{X}$ & $\mathrm{X}$ & $\mathrm{X}$ & $\mathrm{X}$ & $\mathrm{X}$ & 0 \\
\hline 2 & $\mathrm{X}$ & $\mathrm{X}$ & $\mathrm{X}$ & $\mathrm{X}$ & $\mathrm{X}$ & 0 \\
\hline 3 & $\mathrm{X}$ & $\mathrm{X}$ & $\mathrm{X}$ & $\mathrm{X}$ & $\mathrm{X}$ & 0 \\
\hline 4 & $\mathrm{X}$ & $\mathrm{X}$ & $\mathrm{X}$ & $\mathrm{X}$ & $\mathrm{X}$ & 0 \\
\hline 5 & $\mathrm{X}$ & $\mathrm{X}$ & $\mathrm{X}$ & $\mathrm{X}$ & $\mathrm{X}$ & 0 \\
\hline 6 & $\mathrm{X}$ & $\mathrm{X}$ & $\mathrm{X}$ & $\mathrm{X}$ & $\mathrm{X}$ & 0 \\
\hline 7 & $\mathrm{X}$ & $\mathrm{X}$ & $\mathrm{X}$ & $\mathrm{X}$ & $\mathrm{X}$ & 0 \\
\hline 8 & $\mathrm{X}$ & $\mathrm{X}$ & $\mathrm{X}$ & $\mathrm{X}$ & $\mathrm{X}$ & 0 \\
\hline 9 & $\mathrm{X}$ & $\mathrm{X}$ & $\mathrm{X}$ & $\mathrm{X}$ & $\mathrm{X}$ & 0 \\
\hline 10 & $\mathrm{X}$ & $\mathrm{X}$ & $\mathrm{X}$ & $\mathrm{X}$ & $\mathrm{X}$ & 0 \\
\hline
\end{tabular}

\begin{tabular}{|} 
Reproduction at $100.0 \%$ Concentration \\
\hline Rep. & Day 3/4 & Day 5 & Day 6 & Day 7 & Day 8 & Total \\
\hline 1 & & $\mathrm{x}$ & $\mathrm{x}$ & $\mathrm{x}$ & $\mathrm{x}$ & 0 \\
\hline 2 & & $\mathrm{x}$ & $\mathrm{x}$ & $\mathrm{x}$ & $\mathrm{x}$ & 0 \\
\hline 3 & & $\mathrm{x}$ & $\mathrm{x}$ & $\mathrm{x}$ & $\mathrm{x}$ & 0 \\
\hline 4 & & $\mathrm{x}$ & $\mathrm{x}$ & $\mathrm{x}$ & $\mathrm{x}$ & 0 \\
\hline 5 & & $\mathrm{x}$ & $\mathrm{x}$ & $\mathrm{x}$ & $\mathrm{x}$ & 0 \\
\hline 6 & & $\mathrm{x}$ & $\mathrm{x}$ & $\mathrm{x}$ & $\mathrm{x}$ & 0 \\
\hline 7 & & $\mathrm{x}$ & $\mathrm{x}$ & $\mathrm{x}$ & $\mathrm{x}$ & 0 \\
\hline 8 & & $\mathrm{x}$ & $\mathrm{x}$ & $\mathrm{x}$ & $\mathrm{x}$ & 0 \\
\hline 9 & & $\mathrm{x}$ & $\mathrm{x}$ & $\mathrm{x}$ & $\mathrm{x}$ & 0 \\
\hline 10 & & $\mathrm{x}$ & $\mathrm{x}$ & $\mathrm{x}$ & $\mathrm{x}$ & 0 \\
\hline
\end{tabular}




\section{CHRONIC DEFINITIVE SURVIVAL AND REPRODUCTION TEST \\ Statistical Analyses}

Client: Westinhouse Savanah River Company

Sample Identification: A-003

T5970

Test Date: $\quad$ 6/25/96

\begin{tabular}{|lrr|}
\hline Test for Normality & & \\
Test Used: Shapiro Wilks & W= & 0.848 \\
& critical & 0.9 \\
& & \\
The data are not normal in distribution \\
\hline
\end{tabular}

\section{Test for Homogeneity of Variance}

Test Used: Bartlett's Test

$$
\begin{array}{rr}
\mathrm{B}= & 3.22 \\
\text { critical } & 9.21
\end{array}
$$

\begin{tabular}{|c|c|c|c|c|c|c|}
\hline \multicolumn{7}{|c|}{ Test for Differences in Reproduction } \\
\hline \multirow{2}{*}{\multicolumn{2}{|c|}{$\begin{array}{l}\text { Test Type Used: } \\
\text { t Test }\end{array}$}} & \multicolumn{5}{|c|}{ Wilcoxon's Test } \\
\hline & & \multicolumn{5}{|c|}{ Test Concentration } \\
\hline & \begin{tabular}{|l|l|} 
Control \\
\end{tabular} & $6.2 \%$ & $12.5 \%$ & $25 \%$ & $50 \%$ & $100 \%$ \\
\hline \multicolumn{2}{|c|}{ t Value } & 2.56 & \begin{tabular}{|l|l|} 
N/A \\
\end{tabular} & N/A & $\mathrm{N} / \mathrm{A}$ & N/A \\
\hline \multicolumn{2}{|l|}{ critical } & 2.33 & 2.33 & 2.33 & 2.33 & \\
\hline mean & 19.3 & 9.0 & 0.0 & 0.0 & 0.0 & 0.0 \\
\hline \multicolumn{7}{|c|}{ MSD $=9.38$} \\
\hline \multicolumn{7}{|c|}{ Test Concentration } \\
\hline & & 0.062 & 0.125 & 0.25 & 0.5 & 1 \\
\hline \multicolumn{2}{|c|}{ Rank Sum } & 76 & N/A & N/A - & N/A & $\mathrm{N} / \mathrm{A}$ \\
\hline \multicolumn{2}{|c|}{ critical } & 82 & & & & \\
\hline \multicolumn{3}{|c|}{$\begin{array}{l}\text { NOEC (Reproduction) } \\
\text { LOEC (Reproduction) }\end{array}$} & \multicolumn{4}{|c|}{$\begin{array}{l}<6.25 \% \text { effluent } \\
<6.25 \% \text { effluent }\end{array}$} \\
\hline
\end{tabular}

The data are homogeneous in variance

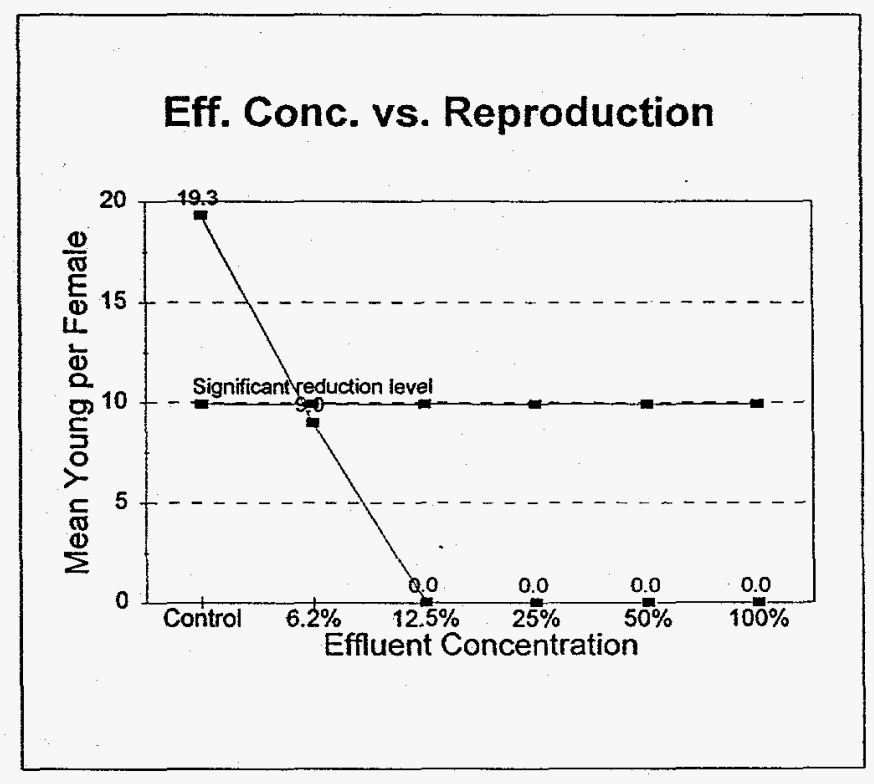




\section{CHRONIC DEFINITIVE SURVIVAL AND REPRODUCTION TEST Water Chemistry}

Client: Westinghouse Savannah River Company

Sample Identification: A-003

Test Date: $\quad$ 6/25/96

pH (After Renewal)

\begin{tabular}{|l|r|r|r|r|r|r|}
\hline \multicolumn{7}{|c|}{ Test Concentration } \\
\hline & Control & $6.25 \%$ & $12.5 \%$ & $25 \%$ & $50.0 \%$ & $100 \%$ \\
\hline Initial & 7.86 & 7.66 & 7.55 & 7.54 & 7.67 & 7.89 \\
\hline Day 1 & 7.42 & 7.34 & 7.25 & 7.23 & & \\
\hline Day 2 & 7.76 & 7.84 & 7.76 & & & \\
\hline Day 3 & 7.00 & 7.22 & 7.16 & & & \\
\hline Day 4 & 7.60 & 7.47 & 7.50 & & & \\
\hline Day 5 & 8.01 & 7.70 & 7.63 & & & \\
\hline Day 6 & 7.90 & 7.85 & 8.14 & & & \\
\hline
\end{tabular}

Dissolved Oxygen (After Renewal)

\begin{tabular}{|l|r|r|r|r|r|r|}
\hline \multicolumn{7}{|c|}{ Test Concentration } \\
\hline & Control & $6.25 \%$ & $12.5 \%$ & $25 \%$ & $50.0 \%$ & $100 \%$ \\
\hline Initial & 9.1 & 8.8 & 8.8 & 8.6 & 8.6 & 8.6 \\
\hline Day 1 & 9.1 & 8.9 & 8.8 & & & \\
\hline Day 2 & 8.6 & 8.8 & 8.8 & & & \\
\hline Day 3 & 8.8 & 8.7 & 8.8 & & & \\
\hline Day 4 & 8.8 & 8.8 & 8.6 & & & \\
\hline Day 5 & 8.9 & 8.8 & 8.8 & & & \\
\hline Day 6 & 8.8 & 8.8 & & & & \\
\hline
\end{tabular}

\section{Dilution Water \& Effluent Parameters}

\begin{tabular}{|l|r|r|r|r|l|}
\hline & Res Cl. & Alkalin & Hard. & Cond. & \\
\hline & $\mathrm{mg} / \mathrm{L}$ & $\mathrm{mg} / \mathrm{L}$ & $\mathrm{mg} / \mathrm{L}$ & $\mu \mathrm{mhos}$ & \\
\hline Eff. Sample 1 & 0.05 & 43.3 & 10 & 1290 & \\
\hline Eff. Sample 2 & 0.5 & 47.4 & 14 & 135.4 & \\
\hline Eff. Sample 3 & 0.1 & 55.6 & 10 & 148.3 & \\
\hline & & & & & \\
\hline Dilution Water & & 70 & 84 & 187.9 & \\
\hline & & & & & \\
\hline & & & & & \\
\hline
\end{tabular}

$\mathrm{pH}$ (Before Renewal)

\begin{tabular}{|l|r|r|r|r|r|r|}
\hline & \multicolumn{7}{|c|}{ Test Concentration } \\
\hline & Control & $6.25 \%$ & $12.5 \%$ & $25 \%$ & $50.0 \%$ & $100 \%$ \\
\hline Day 1 & 7.18 & 7.21 & 7.23 & 7.32 & 7.39 & 7.63 \\
\hline Day 2 & 7.86 & 7.12 & 7.94 & & & \\
\hline Day 3 & 7.16 & 7.24 & 7.29 & & & \\
\hline Day 4 & 8.35 & 8.25 & 8.31 & & & \\
\hline Day 5 & 7.67 & 7.41 & 7.44 & & & \\
\hline Day 6 & 8.06 & 7.97 & & & & \\
\hline Final & 7.89 & 7.83 & 8.14 & & & \\
\hline
\end{tabular}

Dissolved Oxygen (Before Renewal)

\begin{tabular}{|l|r|r|r|r|r|r|}
\hline \multicolumn{7}{|c|}{ Test Concentration } \\
\hline & Control & $6.25 \%$ & $12.5 \%$ & $25 \%$ & $50.0 \%$ & $100 \%$. \\
\hline Day 1 & 8.8 & 8.6 & 8.5 & 8.5 & 8.4 & 8.4 \\
\hline Day 2 & 8.6 & 8.6 & 8.6 & & & \\
\hline Day 3 & 8.6 & 8.6 & 8.6 & & & \\
\hline Day 4 & 8.4 & 8.6 & 8.6 & & & \\
\hline Day 5 & 8.7 & 8.7 & 8.7 & & & \\
\hline Day 6 & 8.6 & 8.7 & & & & \\
\hline Final & & 7.9 & 7.9 & & & \\
\hline
\end{tabular}

Temperature

\begin{tabular}{|l|r|}
\hline \multicolumn{2}{|c|}{ Incubator ${ }^{\circ} \mathrm{C}$} \\
\hline Initial & 24.6 \\
\hline Day 1 & 24.6 \\
\hline Day 2 & 24.9 \\
\hline Day 3 & 24.1 \\
\hline Day 4 & 25.7 \\
\hline Day 5 & 24.7 \\
\hline Day 6 & 25.2 \\
\hline Final & 24.6 \\
\hline
\end{tabular}




\section{Day Chronic Definitive Survival and Reproduction Bioassay}

Method: EPA/600/4-89/001

Test Organism:

Ceriodaphnia dubia

Facility:Westinghouse Savannah River Company

Sample ID:A-004

Date: $\quad 7-09-96$ 


\section{CHRONIC DEFINITIVE SURVIVAL AND REPRODUCTION TEST \\ Survival and Reproduction Results}

Client: Westinghouse Savannah River Company

Sample Identification: A-004

Test Date: $\quad$ 7-09-96

\begin{tabular}{|c|c|c|c|c|c|c|c|}
\hline \multicolumn{3}{|c|}{ Reproduction at } & \multicolumn{7}{c|}{ Concentration } \\
\hline Rep. & Day 2/3 & Day 4 & Day 5 & Day 6 & Day 7 & Day 8 & Total \\
\hline 1 & 0 & 5 & 8 & 11 & 0 & 0 & 24 \\
\hline 2 & 0 & 5 & 6 & 16 & 0 & 0 & 27 \\
\hline 3 & 0 & 3 & 1 & 12 & 12 & 0 & 28 \\
\hline 4 & 0 & 0 & 6 & 0 & 9 & 10 & 25 \\
\hline 5 & 0 & 3 & 10 & 12 & 0 & 0 & 25 \\
\hline 6 & 0 & 0 & 7 & 12 & 1 & 0 & 20 \\
\hline 7 & 0 & 0 & 11 & 13 & 0 & 0 & 24 \\
\hline 8 & 3 & $X$ & $X$ & $X$ & $X$ & $X$ & 3 \\
\hline 9 & 0 & 0 & 9 & 14 & 0 & 0 & 23 \\
\hline 10 & 0 & 3 & 7 & 7 & 0 & 0 & 17 \\
\hline
\end{tabular}

\begin{tabular}{|c|c|c|c|c|c|c|c|}
\hline \multicolumn{6}{|c|}{ Reproduction at } & $6.25 \%$ & \multicolumn{3}{c|}{ Concentration } \\
\hline Rep. & Day 2/3 & Day 4 & Day 5 & Day 6 & Day 7 & Day 8 & Total \\
\hline 1 & 3 & 7 & 0 & 13 & 0 & 0 & 23 \\
\hline 2 & 0 & 3 & 5 & 11 & 0 & 0 & 19 \\
\hline 3 & 2 & 9 & 0 & 0 & 12 & 0 & 23 \\
\hline 4 & 0 & 1 & 3 & 8 & 0 & 3 & 15 \\
\hline 5 & 0 & 1 & 9 & 10 & 0 & 0 & 20 \\
\hline 6 & $X$ & $X$ & $X$ & $X$ & $X$ & $X$ & 0 \\
\hline 7 & 0 & 3 & 3 & 0 & 0 & 10 & 16 \\
\hline 8 & 0 & 0 & 6 & 11 & 5 & 0 & 22 \\
\hline 9 & 0 & 2 & 9 & 12 & 0 & 0 & 23 \\
\hline 10 & 0 & 0 & 0 & 8 & 0 & 9 & 17 \\
\hline
\end{tabular}

\begin{tabular}{|c|c|c|c|c|c|c|c|}
\hline \multicolumn{2}{|c|}{ Reproduction at } & $12.5 \%$ & \multicolumn{3}{c|}{ Concentration } \\
\hline Rep. & Day $2 / 3$ & Day 4 & Day 5 & Day 6 & Day 7 & Day 8 & Total \\
\hline 1 & 4 & 8 & 0 & 10 & 0 & 0 & 22 \\
\hline 2 & 0 & 4 & 6 & 12 & 0 & 0 & 22 \\
\hline 3 & 0 & 4 & 6 & 9 & 0 & 0 & .19 \\
\hline 4 & 2 & 0 & 3 & 13 & 7 & 0 & 25 \\
\hline 5 & 0 & 1 & 6 & 10 & 0 & 9 & 26 \\
\hline 6 & 0 & $\mathrm{X}$ & $\mathrm{X}$ & $\mathrm{X}$ & $\mathrm{X}$ & $\mathrm{X}$ & 0 \\
\hline 7 & 0 & 6 & 0 & 14 & 0 & 0 & 20 \\
\hline 8 & 3 & 0 & 6 & 8 & 0 & 0 & 17 \\
\hline 9 & 0 & 5 & 5 & 12 & 0 & 0 & 22 \\
\hline 10 & 0 & 0 & 5 & 0 & 0 & 0 & 5 \\
\hline
\end{tabular}

\begin{tabular}{|c|c|c|c|c|c|c|c|}
\hline \multicolumn{3}{|c|}{ Reproduction at } & $25.0 \%$ & \multicolumn{1}{c|}{ Concentration } \\
\hline Rep. & Day 2/3 & Day 4 & Day 5 & Day 6 & Day 7 & Day 8 & Total \\
\hline 1 & 3 & 0 & 3 & 9 & 0 & 7 & 22 \\
\hline 2 & 0 & 2 & 3 & 12 & 0 & 0 & 17 \\
\hline 3 & 2 & 6 & 0 & 6 & 9 & 0 & 23 \\
\hline 4 & 0 & 4 & 7 & 9 & 0 & 0 & 20 \\
\hline 5 & 0 & 4 & 9 & 13 & 0 & 0 & 26 \\
\hline 6 & 0 & 4 & 5 & 0 & 8 & 0 & 17 \\
\hline 7 & 0 & 2 & 6 & 12 & 0 & 0 & 20 \\
\hline 8 & 3 & 0 & 6 & 10 & 0 & 0 & 19 \\
\hline 9 & 0 & 3 & 1 & 0 & 11 & 0 & 15 \\
\hline 10 & 0 & 4 & 6 & 10 & 0 & 0 & 20 \\
\hline
\end{tabular}

\begin{tabular}{|c|c|c|c|c|c|c|c|}
\hline \multicolumn{1}{|c|}{ Reproduction at } \\
\hline Rep. & Day 2/3 & Day 4 & Day 5 & Day 6 & Day 7 & Day 8 & Total \\
\hline 1 & 2 & 8 & 0 & 8 & 0 & 0 & 18 \\
\hline 2 & 0 & 2 & 6 & 0 & 10 & 0 & 18 \\
\hline 3 & 3 & 6 & 0 & 8 & 0 & 0 & 17 \\
\hline 4 & $\mathrm{~L}$ & $\mathrm{~L}$ & $\mathrm{~L}$ & $\mathrm{~L}$ & $\mathrm{~L}$ & $\mathrm{~L}$ & $\mathrm{~L}$ \\
\hline 5 & 0 & 4 & 0 & 3 & 0 & 0 & 7 \\
\hline 6 & 0 & 1 & 5 & 0 & 2 & 0 & 8 \\
\hline 7 & $\mathrm{X}$ & $\mathrm{X}$ & $\mathrm{X}$ & $\mathrm{X}$ & $\mathrm{X}$ & $\mathrm{X}$ & 0 \\
\hline 8 & 0 & 4 & 3 & $\mathrm{X}$ & $\mathrm{X}$ & $\mathrm{X}$ & 7 \\
\hline 9 & 0 & 2 & 4 & 7 & 0 & 0 & 13 \\
\hline 10 & $\mathrm{X}$ & $\mathrm{X}$ & $\mathrm{X}$ & $\mathrm{X}$ & $\mathrm{X}$ & $\mathrm{X}$ & 0 \\
\hline
\end{tabular}

\begin{tabular}{|c|c|c|c|c|c|c|c|}
\hline \multicolumn{3}{|c|}{ Reproduction at } & \multirow{2}{*}{$\frac{100.0 \%}{\text { Day } 5}$} & \multicolumn{4}{|c|}{ Concentration } \\
\hline Rep. & Day $2 / 3$ & Day 4 & & Day 6 & Day? & Day 8 & Total \\
\hline 1 & $X$ & $\mathrm{X}$ & $X$ & $x$ & $x$ & $\mathrm{X}$ & 0 \\
\hline 2 & $\bar{x}$ & $x$ & $x$ & $x$ & $\mathrm{X}$ & $\bar{x}$ & 0 \\
\hline 3 & $\bar{x}$ & $x$ & $x$ & $x$ & $X$ & $x$ & 0 \\
\hline 4 & $\bar{X}$ & X & $\bar{x}$ & $\bar{x}$ & $X$ & $\bar{X}$ & 0 \\
\hline 5 & $\bar{X}$ & $x$ & $\mathrm{X}$ & $x$ & $x$ & $\mathrm{X}$ & 0 \\
\hline 6 & $x$ & $x$ & $\mathrm{X}$ & $x$ & $\mathrm{X}$ & $X$ & 0 \\
\hline 7 & $\bar{x}$ & $x$ & $\bar{x}$ & $x$ & 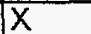 & $\bar{x}$ & 0 \\
\hline 8 & $x$ & $x$ & $\bar{X}$ & $x$ & $\mathrm{X}$ & $x$ & 0 \\
\hline 9 & $\bar{x}$ & $X$ & $\mathrm{X}$ & $x$ & $x$ & $x$ & 0 \\
\hline 10 & $x$ & $\mathrm{X}$ & $\mathrm{X}$ & $x$ & $\mathrm{X}$ & $\mathrm{X}$ & 0 \\
\hline
\end{tabular}

\footnotetext{
$X=$ Dead

$L=$ Lost or caught in surface tension of water
} 


\section{CHRONIC DEFINITIVE SURVIVAL AND REPRODUCTION TEST \\ Statistical Analyses}

Client: Westinghouse Savannah River Company

Sample Identification: A-004

Test Date: 7-09-96

\section{Test for Normality}

Test Used: Shapiro Wilks

$$
\begin{array}{rr}
\mathrm{W}= & 0.863 \\
\text { critical } & 0.93
\end{array}
$$

The data are not normal in distribution

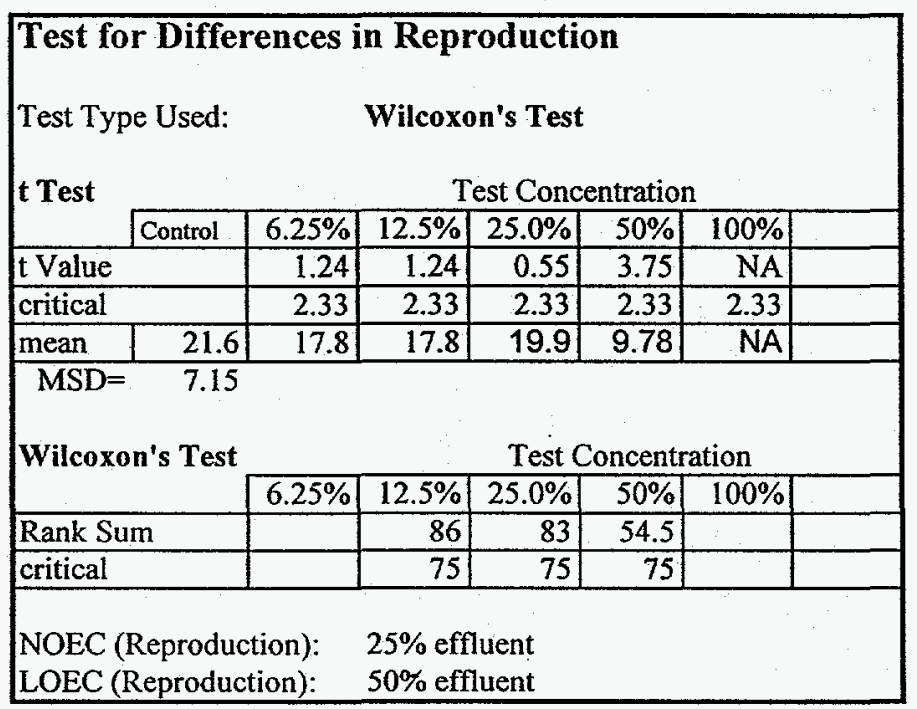

\section{Test for Homogeneity of Variance}

Test Used: Bartlett's Test

$$
\mathrm{B}=
$$$$
\text { critical }
$$

7.77

13.28

The data are homogeneous in variance

\section{Effluent Conc. vs. Reproduction}

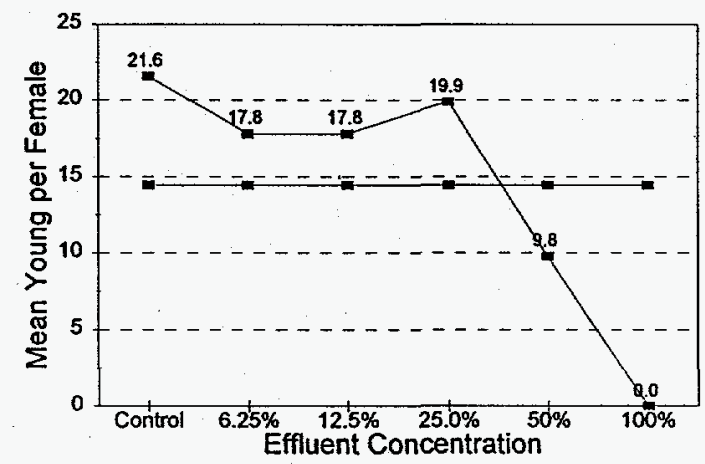

\section{Summary}

The effluent is toxic at concentrations of $50 \%$ and higher. 


\section{CHRONIC DEFINITIVE SURVIVAL AND REPRODUCTION TEST \\ Water Chemistry}

Client: Westinghouse Savannah River Company

Sample Identification: A-004

Test Date: $\quad$ 7-09-96

pH (After Renewal)

\begin{tabular}{|c|c|c|c|c|c|c|}
\hline & \multicolumn{6}{|c|}{ Test Concentration } \\
\hline & Control & $6.25 \%$ & $12.5 \%$ & $25.0 \%$ & $50 \%$ & $100 \%$ \\
\hline Initial & 6.60 & 7.30 & 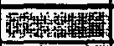 & 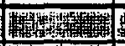 & 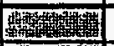 & 7.30 \\
\hline Day 1 & 6.57 & 7.94 & 4⿹ & 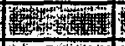 & 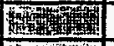 & 7.94 \\
\hline Day 2 & 6.54 & 7.72 & Hen & 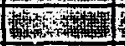 & 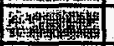 & 7.72 \\
\hline Day 3 & 7.05 & 8.21 & 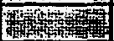 & 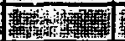 & \begin{tabular}{|c|}
4 \\
4
\end{tabular} & 8.21 \\
\hline Day 4 & no data & 8.37 & 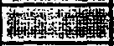 & 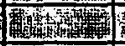 & 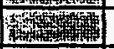 & 8.37 \\
\hline Day 5 & 6.47 & 7.86 & 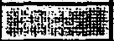 & 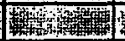 & 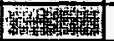 & 7.86 \\
\hline Day 6 & 6.58 & 8.21 & 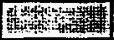 & 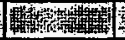 & 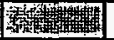 & 8.21 \\
\hline
\end{tabular}

\section{Dissolved Oxygen (After Renewal)}

\begin{tabular}{|c|c|c|c|c|c|c|c|}
\hline & \multicolumn{7}{|c|}{ Test Concentration } \\
\hline & Control & $6.25 \%$ & $12.5 \%$ & $25.0 \%$ & $50 \%$ & $100 \%$ & \\
\hline Initial & 7.9 & 8.2 & Why & Thy & 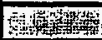 & 7.8 & \\
\hline Day 1 & 8.2 & 8.4 & ow & 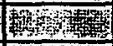 & Wy & 8.6 & \\
\hline Day 2 & 8.5 & 8.1 & 19 & 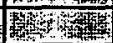 & 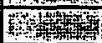 & 8.4 & \\
\hline Day 3 & 8.1 & 8.3 & 34 & $47^{164}$ & 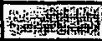 & 8.4 & \\
\hline Day 4 & no data & no data & maty & 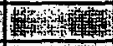 & 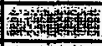 & no data & \\
\hline Day 5 & 8.6 & 8.5 & $\sqrt{1+4}$ & मी. & 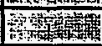 & 8.4 & \\
\hline Day 6 & 8.5 & 8.4 & PithM & Wमा & $\sqrt{3+14}$ & & \\
\hline
\end{tabular}

\section{Dilution Water \& Effluent Parameters}

\begin{tabular}{|l|c|r|r|r|l|l|}
\hline & Res Cl. & Alkalin & Hard. & Cond. & & \\
\hline & $\mathrm{mg} / \mathrm{L}$ & $\mathrm{mg} / \mathrm{L}$ & $\mathrm{mg} / \mathrm{L}$ & $\mu \mathrm{mhos}$ & & \\
\hline Eff. Sample 1 & $<.01$ & 26.8 & 2.0 & 79.1 & & \\
\hline Eff. Sample 2 & .10 & 57.7 & 4.0 & 140 & & \\
\hline Eff. Sample 3 & .20 & 57.7 & 4.0 & 131 & & \\
\hline & & & & & & \\
\hline & & & & & & \\
\hline & & & & & & \\
\hline & & & & & & \\
\hline
\end{tabular}

pH (Before Renewal)

\begin{tabular}{|c|c|c|c|c|c|c|}
\hline & \multicolumn{6}{|c|}{ Test Concentration } \\
\hline & Control & $6.25 \%$ & $12.5 \%$ & $25.0 \%$ & $50 \%$ & $100 \%$ \\
\hline Day 1 & 7.29 & 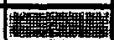 & T) & (1) & 样 & (1) \\
\hline Day 2 & 8.01 & 11 & $=10$ & 10 & 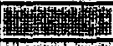 & 1) \\
\hline Day 3 & 7.92 & $\sqrt{4}$ & (1) & 15y & 7\% & (1) \\
\hline Day 4 & & 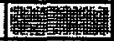 & 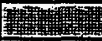 & 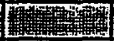 & 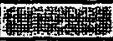 & (1) \\
\hline Day 5 & 8.15 & (1) & 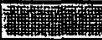 & 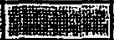 & 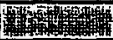 & $1+1+4$ \\
\hline Day 6 & 8.07 & 17 & (n) & 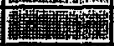 & The & 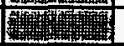 \\
\hline Final & 8.12 & W & (1) & 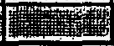 & 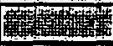 & (1) \\
\hline
\end{tabular}

Dissolved Oxygen (Before Renewal)

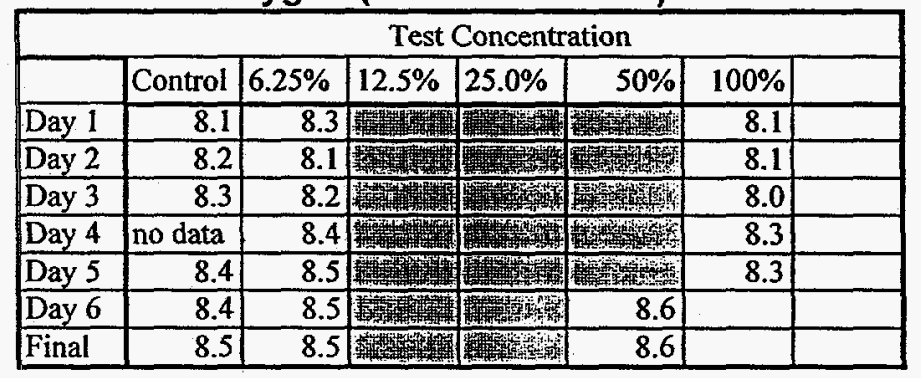

\section{Temperature}

\begin{tabular}{|l|r|}
\hline \multicolumn{2}{|c|}{ Incubator ${ }^{\circ} \mathrm{C}$} \\
\hline Initial & 24.8 \\
\hline Day 1 & 24.0 \\
\hline Day 2 & 24.0 \\
\hline Day 3 & 25.1 \\
\hline Day 4 & 25.2 \\
\hline Day 5 & 24.0 \\
\hline Day 6 & 24.8 \\
\hline Final & 24.3 \\
\hline
\end{tabular}


(864) 877-6942 - FAX (864) 877-6938

P.O. Box 16414, Greenville, SC 29606 - 4 Craftsman Court, Greer, SC 29650

\section{Day Chronic Definitive Survival and Reproduction Bioassay \\ Method: EPA/600/4-89/001}

Test Organism:

Ceriodaphnia dubia

Facility:Westinghouse Savannah River Company

Sample ID:A-005

Date: $\quad 7 / 09 / 96$ 


\section{CHRONIC DEFINITIVE SURVIVAL AND REPRODUCTION TEST Survival and Reproduction Results}

Client: Westinghouse Savannah River Company

Sample Identification: A-005

Test Date: $\quad 7 / 09 / 96$

\begin{tabular}{|c|c|c|c|c|c|c|c|}
\hline \multicolumn{3}{|c|}{ Reproduction at } & \multirow{2}{*}{$\frac{0.0 \%}{\text { Day } 5}$} & \multicolumn{4}{|c|}{ Concentration } \\
\hline Rep. & Day $2 / 3$ & Day 4 & & Day 6 & Day 7 & Day 8 & Total \\
\hline 1 & 0 & 5 & 8 & 11 & 0 & 0 & 24 \\
\hline 2 & 0 & 5 & 6 & 16 & 0 & 0 & 27 \\
\hline 3 & 0 & 3 & 1 & 12 & 12 & 0 & 28 \\
\hline 4 & 0 & 0 & 6 & 0 & 9 & 0 & 15 \\
\hline 5 & 0 & 3 & 10 & 12 & 0 & 0 & 25 \\
\hline 6 & 0 & 0 & 7 & 12 & 1 & 0 & 20 \\
\hline 7 & 0 & 0 & 11 & 13 & 0 & 0 & 24 \\
\hline 8 & 3 & $x$ & $x$ & $x$ & $x$ & $x$ & 3 \\
\hline 9 & 0 & 0 & 9 & 14 & 0 & 0 & 23 \\
\hline 10 & 0 & 3 & 7 & 0 & 0 & 0 & 10 \\
\hline
\end{tabular}

\begin{tabular}{|c|c|c|c|c|c|c|c|}
\hline \multicolumn{9}{|c|}{ Reproduction at } & $6.25 \%$ & \multicolumn{1}{c|}{ Concentration } \\
\hline Rep. & Day 2/3 & Day 4 & Day 5 & Day 6 & Day 7 & Day 8 & Total \\
\hline 1 & 3 & 0 & 0 & 0 & 0 & 5 & 8 \\
\hline 2 & 5 & 0 & 0 & 8 & 9 & 0 & 22 \\
\hline 3 & 5 & 0 & 8 & 13 & 0 & 0 & 26 \\
\hline 4 & 4 & 8 & 0 & 8 & 0 & 0 & 20 \\
\hline 5 & 3 & $\times$ & $\times$ & $x$ & $x$ & $\times$ & 3 \\
\hline 6 & 2 & 4 & 0 & 11 & 0 & 2 & 19 \\
\hline 7 & 0 & 3 & 2 & 0 & 5 & 5 & 15 \\
\hline 8 & 0 & 0 & 1 & 0 & 7 & 7 & 15 \\
\hline 9 & 0 & 2 & 9 & 12 & 0 & 0 & 23 \\
\hline 10 & 5 & 8 & 0 & 15 & 0 & 0 & 28 \\
\hline
\end{tabular}

\begin{tabular}{|c|c|c|c|c|c|c|c|}
\hline \multicolumn{2}{|c|}{ Reproduction at } & $12.5 \%$ & \multicolumn{1}{c|}{ Concentration } \\
\hline Rep. & Day 2/3 & Day 4 & Day 5 & Day 6 & Day 7 & Day 8 & Total \\
\hline 1 & 0 & 4 & 3 & 7 & 0 & 0 & 14 \\
\hline 2 & 0 & 7 & 0 & 0 & 11 & 0 & 18 \\
\hline 3 & 4 & 0 & 5 & 7 & 8 & 0 & 24 \\
\hline 4 & 4 & 4 & 0 & 14 & 0 & 0 & 22 \\
\hline 5 & 3 & 0 & 5 & 10 & 0 & 0 & 18 \\
\hline 6 & 1 & 6 & 0 & 13 & 0 & 0 & 20 \\
\hline 7 & 0 & 2 & 3 & 14 & 0 & 0 & 19 \\
\hline 8 & 0 & 5 & 7 & 0 & 11 & 0 & 23 \\
\hline 9 & 0 & 5 & 5 & 0 & 8 & 0 & 18 \\
\hline 10 & 4 & 0 & 0 & 19 & 0 & 0 & 23 \\
\hline
\end{tabular}

\begin{tabular}{|c|c|c|c|c|c|c|c|}
\hline \multicolumn{8}{|c|}{ Reproduction at } \\
\hline Rep. & Day 2/3 & Day 4 & Day 5 & Day 6 & Day 7 & Day 8 & Total \\
\hline 1 & 1 & 0 & 0 & 7 & 9 & 0 & 17 \\
\hline 2 & 0 & 7 & 0 & 10 & 8 & 0 & 25 \\
\hline 3 & 4 & 0 & 6 & 9 & 0 & 0 & 19 \\
\hline 4 & 4 & 0 & 0 & 11 & 3 & 0 & 18 \\
\hline 5 & 3 & 3 & 6 & 11 & 0 & 0 & 23 \\
\hline 6 & 0 & 3 & 0 & 1 & 12 & 9 & 25 \\
\hline 7 & 0 & 3 & 5 & 16 & 0 & 0 & 24 \\
\hline 8 & 4 & 0 & 0 & 7 & 11 & 0 & 22 \\
\hline 9 & 0 & 3 & 3 & 12 & 0 & 13 & 31 \\
\hline 10 & 3 & 0 & 7 & 15 & 0 & 0 & 25 \\
\hline
\end{tabular}

\begin{tabular}{|c|c|c|c|c|c|c|c|}
\hline \multicolumn{7}{|c|}{ Reproduction at } & \multicolumn{7}{l|}{ Concentration } \\
\hline Rep. & Day 2/3 & Day 4 & Day 5 & Day 6 & Day 7 & Day 8 & Total \\
\hline 1 & 0 & 0 & 4 & 6 & 0 & 9 & 19 \\
\hline 2 & 0 & 5 & 0 & 2 & 6 & 7 & 20 \\
\hline 3 & 0 & 0 & 7 & 11 & 4 & 0 & 22 \\
\hline 4 & 0 & 0 & 1 & 1 & 0 & 10 & 12 \\
\hline 5 & 0 & 3 & 8 & 3 & 0 & 11 & 25 \\
\hline 6 & 0 & 0 & 0 & 2 & 0 & 1 & 3 \\
\hline 7 & 0 & 0 & 5 & 18 & 0 & 0 & 23 \\
\hline 8 & 0 & 0 & 5 & 0 & 9 & 9 & 23 \\
\hline 9 & 0 & 3 & 4 & 5 & 0 & 14 & 26 \\
\hline 10 & 3 & 0 & 6 & 3 & 8 & 0 & 20 \\
\hline
\end{tabular}

\begin{tabular}{|c|c|c|c|c|c|c|c|}
\hline \multicolumn{3}{|c|}{ Reproduction at } & \multicolumn{3}{|c|}{$100.0 \%$ Concentration } & \multirow[b]{2}{*}{ Day 8} & \multirow[b]{2}{*}{ Total } \\
\hline Rep. & Day $2 / 3$ & Day 4 & Day 5 & Day 6 & Day 7 & & \\
\hline 1 & $X$ & $\mathrm{X}$ & $x$ & $\mathrm{x}$ & $x$ & $x$ & 0 \\
\hline 2 & $\bar{x}$ & $\bar{X}$ & $\bar{x}$ & $\bar{X}$ & $\bar{x}$ & $x$ & 0 \\
\hline 3 & $\bar{x}$ & $\bar{X}$ & $\bar{x}$ & $\bar{x}$ & $\bar{x}$ & $\bar{x}$ & $\overline{0}$ \\
\hline 4 & $\bar{X}$ & $\bar{X}$ & $\bar{x}$ & $\bar{x}$ & $x$ & $\bar{x}$ & 0 \\
\hline 5 & $\bar{x}$ & $\bar{X}$ & $\bar{x}$ & $\bar{X}$ & $\bar{X}$ & $\bar{x}$ & $\overline{0}$ \\
\hline 6 & $\bar{X}$ & $\bar{x}$ & $\bar{x}$ & $\bar{x}$ & $\bar{X}$ & $x$ & 0 \\
\hline 7 & $\mathrm{X}$ & $x$ & $x$ & $x$ & $x$ & $\mathrm{X}$ & 0 \\
\hline 8 & $\bar{x}$ & $\bar{X}$ & $\mathrm{X}$ & $\mathrm{X}$ & $\bar{x}$ & $\bar{x}$ & 0 \\
\hline 9 & $X$ & $x$ & $x$ & $\mathrm{X}$ & $x$ & $\bar{x}$ & 0 \\
\hline 10 & $\bar{x}$ & $\bar{x}$ & $\bar{x}$ & $\bar{x}$ & $\bar{x}$ & $\bar{x}$ & 0 \\
\hline
\end{tabular}

$X=$ Dead

$L=$ Lost or caught in surface tension of water 


\section{CHRONIC DEFINITIVE SURVIVAL AND REPRODUCTION TEST \\ Statistical Analyses}

Client: Westinghouse Savannah River Company

Sample Identification: A-005

Test Date: $\quad 7 / 09 / 96$

\section{Test for Normality}

Test Used: Shapiro Wilks

$$
\begin{array}{r}
\mathrm{W}= \\
\text { critical }
\end{array}
$$

0.93

\section{Test for Homogeneity of Variance}

Test Used: Bartlett's Test

$$
\begin{array}{r}
\mathrm{B}= \\
\text { critical }
\end{array}
$$

10.80

13.28

The data are homogeneous in variance

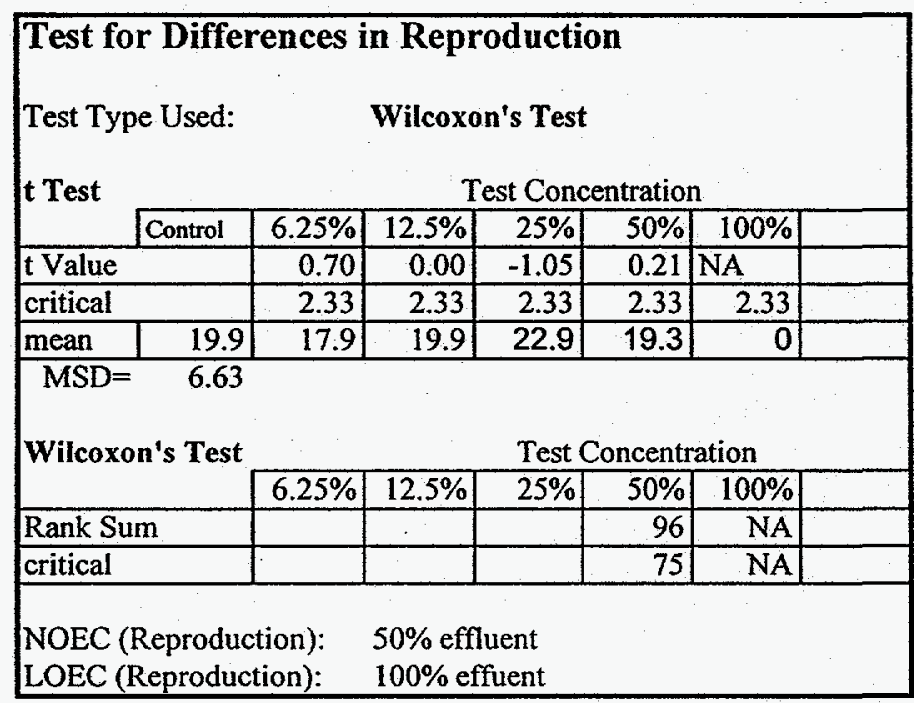

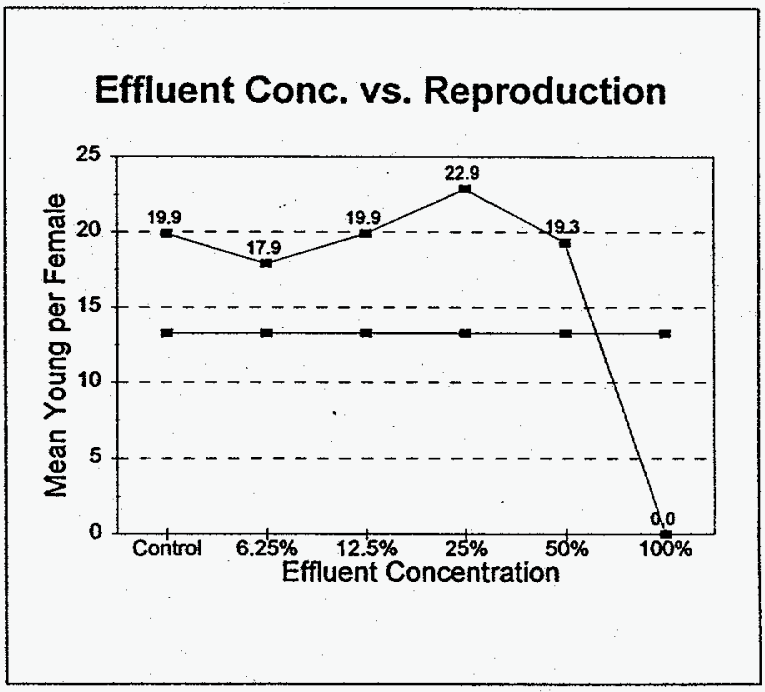

\footnotetext{
Summary

The effluent is toxic at $100 \%$ concentration but not at $50 \%$ concentration.
} 


\section{CHRONIC DEFINITIVE SURVIVAL AND REPRODUCTION TEST \\ Water Chemistry}

Client: Westinghouse Savannah River Company

Sample Identification: A-005

Test Date: $\quad$ 7/09/96

pH (After Renewal)

\begin{tabular}{|c|c|c|c|c|c|c|c|}
\hline & \multicolumn{7}{|c|}{ Test Concentration } \\
\hline & Control & $6.25 \%$ & $12.5 \%$ & $25 \%$ & $50 \%$ & $100 \%$ & \\
\hline Initial & 6.60 & 7.24 & 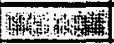 & 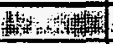 & 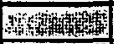 & 7.72 & 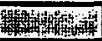 \\
\hline Day 1 & 6.57 & 7.72 & 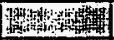 & 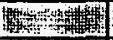 & 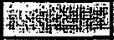 & 7.72 & 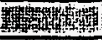 \\
\hline Day 2 & 6.54 & 7.58 & 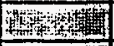 & 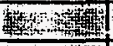 & 17xty & 7.72 & 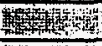 \\
\hline Day 3 & 7.05 & 6.95 & 1) & 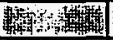 & 新和 & 7.72 & 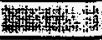 \\
\hline Day 4 & no data & 8.04 & 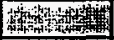 & $4+4 y^{4}$ & 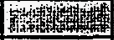 & 7.72 & 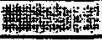 \\
\hline Day 5 & 6.47 & 8.11 & 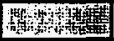 & 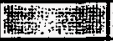 & 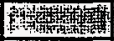 & 7.72 & 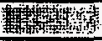 \\
\hline Day 6 & 6.58 & 7.71 & 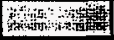 & 稆㷏, & 7.93 & & 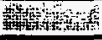 \\
\hline
\end{tabular}

Dissolved Oxygen (After Renewal)

\begin{tabular}{|c|c|c|c|c|c|c|}
\hline & \multicolumn{6}{|c|}{ Test Concentration } \\
\hline & Control & $6.25 \%$ & $12.5 \%$ & $25 \%$ & $50 \%$ & $100 \%$ \\
\hline Initial & 7.9 & 8.0 & 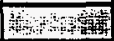 & 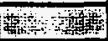 & 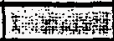 & 8.0 \\
\hline Day 1 & 8.2 & 7.6 & $20+19$ & 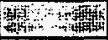 & 1) & 8.0 \\
\hline Day 2 & 8.5 & 8.1 & Artty & 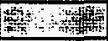 & Hyty & 8.0 \\
\hline Day 3 & 8.1 & 8.0 & 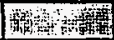 & 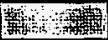 & 4I & 8.0 \\
\hline Day 4 & no data & no data & yty & 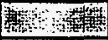 & 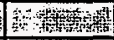 & 8.0 \\
\hline Day 5 & 8.6 & 8.4 & and & 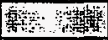 & arternding & 8.0 \\
\hline Day 6 & 8.5 & 8.5 & (t) & 1 & ant? & 8.0 \\
\hline
\end{tabular}

Dilution Water \& Effluent Parameters

\begin{tabular}{|l|r|r|r|r|r|r|}
\hline & Res Cl. & Alkalin & Hard. & Cond. & & \\
\hline & $\mathrm{mg} / \mathrm{L}$ & $\mathrm{mg} / \mathrm{L}$ & $\mathrm{mg} / \mathrm{L}$ & $\mu \mathrm{mhos}$ & & \\
\hline Eff. Sample 1 & 0.16 & 35 & 4.0 & 95 & & \\
\hline Eff. Sample 2 & 0.17 & 59.7 & 6.0 & 139 & & \\
\hline Eff. Sample 3 & 0.05 & 51.5 & 4.0 & 108 & & \\
\hline & & & & & & \\
\hline Dilution Water & & & & & & \\
\hline & & & & & & \\
\hline & & & & & & \\
\hline
\end{tabular}

pH (Before Renewal)

\begin{tabular}{|c|c|c|c|c|c|c|c|}
\hline & \multicolumn{7}{|c|}{ Test Concentration } \\
\hline & Control & $6.25 \%$ & $12.5 \%$ & $25 \%$ & $50 \%$ & $100 \%$ & \\
\hline Day 1 & 7.29 & 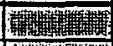 & (1) & 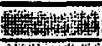 & 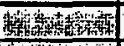 & 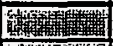 & 19 \\
\hline Day 2 & 8.01 & 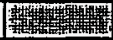 & (1) & 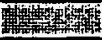 & 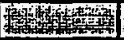 & (1) & II) \\
\hline Day 3 & 7.92 & 19.4 & -14 & Tht & 7) & (14) & (1) \\
\hline Day 4 & no data & (4) & 特) & 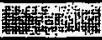 & 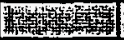 & 1) & 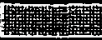 \\
\hline Day 5 & 8.15 & 滆 & 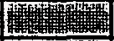 & 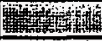 & The & 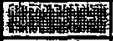 & 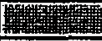 \\
\hline Day 6 & 8.07 & Hin & - in & 1) & 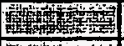 & 玨 & 1) \\
\hline Final & 8.18 & 啃 & 1) & 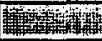 & 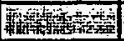 & 14 & II \\
\hline
\end{tabular}

Dissolved Oxygen (Before Renewal)

\begin{tabular}{|c|c|c|c|c|c|c|}
\hline & \multicolumn{6}{|c|}{ Test Concentration } \\
\hline & Control & $6.25 \%$ & $12.5 \%$ & $25 \%$ & $50 \%$ & $100 \%$ \\
\hline Day 1 & 8.1 & 7.8 & 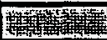 & 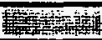 & 1) & 7.5 \\
\hline Day 2 & $\overline{8.2}$ & 8.2 & 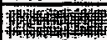 & 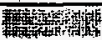 & 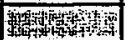 & 7.5 \\
\hline Day 3 & 8.3 & 8.1 & 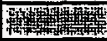 & 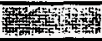 & 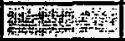 & 7.5 \\
\hline Day 4 & no data & 8.4 & 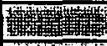 & 1) & 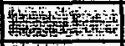 & 7.5 \\
\hline Day 5 & 8.4 & 8.3 & 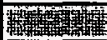 & 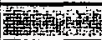 & 17y ty & 7.5 \\
\hline Day 6 & 8.4 & 8.5 & 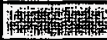 & 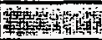 & Hysans & 7.5 \\
\hline Final & & 8.2 & 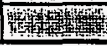 & 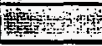 & $\mathrm{DAs}$ & 7.5 \\
\hline
\end{tabular}

Temperature
\begin{tabular}{|l|r|}
\hline Incubator ${ }^{\circ} \mathrm{C}$ \\
\hline Initial & 24.8 \\
\hline Day 1 & 24.0 \\
\hline Day 2 & 24.0 \\
\hline Day 3 & 25.1 \\
\hline Day 4 & 25.2 \\
\hline Day 5 & 24.0 \\
\hline Day 6 & 24.8 \\
\hline Final & 24.3 \\
\hline
\end{tabular}




\section{Day Chronic Definitive Survival and Reproduction Bioassay}

Method: EPA/600/4-89/001

Test Organism:

Ceriodaphnia dubia

Facility:Westinghouse Savannah River Company

Sample ID:A-006

Date: $\quad 7 / 09 / 96$ 


\section{CHRONIC DEFINITIVE SURVIVAL AND REPRODUCTION TEST Survival and Reproduction Results}

Client: Westinghouse Savannah River Company

Sample Identification: A-006

Test Date: $\quad 7 / 09 / 96$

\begin{tabular}{|c|c|c|c|c|c|c|c|}
\hline \multicolumn{1}{|c|}{ Reproduction at } & \multicolumn{1}{|c|}{ Concentration } \\
\hline Rep. & Day 2/3 & Day 4 & Day 5 & Day 6 & Day 7 & Day 8 & Total \\
\hline 1 & 0 & 0 & 0 & 11 & 0 & 4 & 15 \\
\hline 2 & 3 & 0 & 2 & 8 & 12 & 0 & 25 \\
\hline 3 & 0 & 1 & 5 & 5 & 0 & 3 & 14 \\
\hline 4 & 0 & 4 & 8 & 12 & 0 & 0 & 24 \\
\hline 5 & 3 & 0 & 8 & 10 & 0 & 0 & 21 \\
\hline 6 & 2 & 0 & 2 & 7 & 10 & 0 & 21 \\
\hline 7 & 0 & 0 & 7 & 0 & 0 & 0 & 7 \\
\hline 8 & 0 & 0 & 6 & 0 & 14 & 0 & 20 \\
\hline 9 & 2 & 0 & 2 & 10 & 8 & 0 & 22 \\
\hline 10 & 0 & 0 & 6 & 10 & 8 & 0 & 24 \\
\hline
\end{tabular}

\begin{tabular}{|c|c|c|c|c|c|c|c|}
\hline \multicolumn{9}{|c|}{ Reproduction at } & $6.25 \%$ & \multicolumn{1}{c|}{ Concentration } \\
\hline Rep. & Day 2/3 & Day 4 & Day 5 & Day 6 & Day 7 & Day 8 & Total \\
\hline 1 & 0 & 4 & 2 & 7 & 0 & 5 & 18 \\
\hline 2 & 4 & 7 & 0 & 12 & 0 & 0 & 23 \\
\hline 3 & 0 & 0 & 6 & 0 & 0 & 5 & 11 \\
\hline 4 & 0 & 4 & 6 & 10 & 0 & 0 & 20 \\
\hline 5 & 3 & 0 & 8 & 10 & 0 & 0 & 21 \\
\hline 6 & 1 & 0 & 7 & 9 & 0 & 0 & 17 \\
\hline 7 & 3 & 0 & 3 & 15 & 0 & 0 & 21 \\
\hline 8 & 0 & 2 & 4 & 0 & 14 & 0 & 20 \\
\hline 9 & 4 & 0 & 3 & 12 & 0 & 0 & 19 \\
\hline 10 & 3 & 6 & 0 & 13 & 0 & 0 & 22 \\
\hline
\end{tabular}

\begin{tabular}{|c|c|c|c|c|c|c|c|}
\hline \multicolumn{2}{|c|}{ Reproduction at } & $12.5 \%$ & Concentration \\
\hline Rep. & Day 2/3 & Day 4 & Day 5 & Day 6 & Day 7 & Day 8 & Total \\
\hline 1 & 0 & 0 & 6 & 12 & 9 & 0 & 27 \\
\hline 2 & 2 & 9 & 0 & 10 & 0 & 0 & 21 \\
\hline 3 & 0 & 2 & 7 & 12 & 0 & 0 & 21 \\
\hline 4 & 0 & 4 & 6 & 12 & 0 & 0 & 22 \\
\hline 5 & 2 & 0 & 8 & 11 & 0 & 0 & 21 \\
\hline 6 & 0 & 0 & 6 & 9 & 0 & 4 & 19 \\
\hline 7 & 0 & 0 & 4 & 8 & 5 & 0 & 17 \\
\hline 8 & 0 & 6 & 0 & 7 & 14 & 0 & 27 \\
\hline 9 & 0 & 0 & 6 & 10 & 0 & 0 & 16 \\
\hline 10 & 1 & 0 & 9 & 13 & 0 & 0 & 23 \\
\hline
\end{tabular}

\begin{tabular}{|c|c|c|c|c|c|c|c|}
\hline \multicolumn{6}{|c|}{ Reproduction at } & \multicolumn{6}{l|}{ Concentration } \\
\hline Rep. & Day 2/3 & Day 4 & Day 5 & Day 6 & Day 7 & Day 8 & Total \\
\hline 1 & 0 & 3 & 7 & 5 & 0 & 0 & 15 \\
\hline 2 & 5 & 5 & 0 & 9 & 0 & 0 & 19 \\
\hline 3 & 0 & 0 & 3 & 9 & 8 & 0 & 20 \\
\hline 4 & 0 & 5 & 3 & 11 & 0 & 0 & 19 \\
\hline 5 & 0 & 4 & 6 & 2 & 0 & 10 & 22 \\
\hline 6 & 2 & 6 & 0 & 8 & 8 & 0 & 24 \\
\hline 7 & 3 & 0 & 7 & 5 & 5 & 0 & 20 \\
\hline 8 & 0 & 2 & 3 & 0 & 10 & 14 & 29 \\
\hline 9 & 2 & 6 & 0 & 10 & 0 & 0 & 18 \\
\hline 10 & 2 & 9 & 0 & 14 & 0 & 0 & 25 \\
\hline
\end{tabular}

\begin{tabular}{|c|c|c|c|c|c|c|c|}
\hline \multicolumn{8}{|c|}{ Reproduction at } \\
\hline Rep. & Day 2/3 & Day 4 & Day 5 & Day 6 & Day 7 & Day 8 & Total \\
\hline 1 & 0 & 2 & 8 & 13 & 0 & 0 & 23 \\
\hline 2 & 0 & 4 & 0 & 12 & 12 & 0 & 28 \\
\hline 3 & 0 & 4 & 5 & 14 & 0 & 0 & 23 \\
\hline 4 & 0 & 5 & 2 & 11 & 0 & 0 & 18 \\
\hline 5 & 0 & 0 & 8 & 11 & 0 & 0 & 19 \\
\hline 6 & 0 & 8 & 0 & 14 & 0 & 0 & 22 \\
\hline 7 & 0 & 0 & 2 & 15 & 13 & 0 & 30 \\
\hline 8 & 0 & 4 & 3 & $\times$ & $X$ & 0 & 7 \\
\hline 9 & 3 & 0 & 7 & 13 & 0 & 0 & 23 \\
\hline 10 & 1 & 8 & 0 & 9 & 0 & 0 & 18 \\
\hline
\end{tabular}

\begin{tabular}{|c|c|c|c|c|c|c|c|}
\hline \multicolumn{7}{|c|}{ Reproduction at } & \multicolumn{7}{|c|}{ Co.0\% } & \multicolumn{1}{|c|}{ Concentration } \\
\hline Rep. & Day 2/3 & Day 4 & Day 5 & Day 6 & Day 7 & Day 8 & Total \\
\hline 1 & 0 & 4 & 9 & 11 & 0 & 0 & 24 \\
\hline 2 & 1 & 5 & -0 & 10 & 13 & 0 & 29 \\
\hline 3 & 0 & 2 & 7 & 8 & 6 & 0 & 23 \\
\hline 4 & 3 & 0 & 8 & 0 & 17 & 0 & 28 \\
\hline 5 & 2 & 0 & 2 & 6 & 0 & 3 & 13 \\
\hline 6 & 0 & 0 & 5 & 3 & 6 & 5 & 19 \\
\hline 7 & 0 & 0 & 7 & 0 & 0 & 9 & 16 \\
\hline 8 & 0 & 1 & 7 & 0 & 16 & 0 & 24 \\
\hline 9 & 3 & 0 & 3 & 3 & 0 & 4 & 13 \\
\hline 10 & 1 & 8 & 0 & 13 & 0 & 0 & 22 \\
\hline
\end{tabular}

$X=$ Dead

$L=$ Lost or caught in surface tension of water 


\section{CHRONIC DEFINITIVE SURVIVAL AND REPRODUCTION TEST \\ Statistical Analyses}

Client: Westinghouse Savannah River Company

Sample Identification: A-006

Test Date: 7/09/96

Test for Normality

Test Used: Shapiro Wilks

$\begin{array}{rr}\mathrm{W}= & 0.961 \\ \text { critical } & 0.93\end{array}$

The data are normal in distribution

\begin{tabular}{|c|c|c|c|c|c|c|}
\hline \multicolumn{7}{|c|}{ Test for Differences in Reproduction } \\
\hline \multicolumn{3}{|c|}{ Test Type Used: } & \multicolumn{4}{|l|}{ t Test } \\
\hline \multirow[t]{2}{*}{ t Test } & \multicolumn{6}{|c|}{ Test Concentration } \\
\hline & Control & $6.25 \%$ & $12.5 \%$ & $25 \%$ & $50 \%$ & $100 \%$ \\
\hline \multicolumn{2}{|l|}{ t Value } & 0.05 & -0.96 & -0.82 & -0.82 & -0.82 \\
\hline \multicolumn{2}{|l|}{ critical } & 2.33 & 2.33 & 2.33 & 2.33 & 2.33 \\
\hline mean & 19.3 & 19.2 & 21.4 & 21.1 & 21.1 & 21.1 \\
\hline \multicolumn{7}{|c|}{$\mathrm{MSD}=5.09$} \\
\hline \multirow{2}{*}{\multicolumn{2}{|c|}{ Wilcoxon's Test }} & \multicolumn{5}{|c|}{ Test Concentration } \\
\hline & & $6.25 \%$ & $12.5 \%$ & $25 \%$ & $50 \%$ & $100 \%$ \\
\hline \multicolumn{7}{|c|}{ Rank Sum } \\
\hline \multicolumn{2}{|c|}{ critical } & & & & & \\
\hline $\begin{array}{l}\text { NOEC } \\
\text { LOEC }\end{array}$ & $\begin{array}{l}\text { Reprodu } \\
\text { Reproduc }\end{array}$ & $\begin{array}{l}\text { tion): } \\
\text { ion): }\end{array}$ & $\begin{array}{r}100 \% \\
>100 \%\end{array}$ & $\begin{array}{l}\text { fluent } \\
\text { fluent }\end{array}$ & & \\
\hline
\end{tabular}

\begin{tabular}{|ccc|}
\hline \multicolumn{3}{|c|}{ Test for Homogeneity of Variance } \\
Test Used: Bartlett's Test & $\mathrm{B}=$ & 6.09 \\
& critical & 15.1 \\
& & \\
The data are homogeneous in variance & \\
\end{tabular}

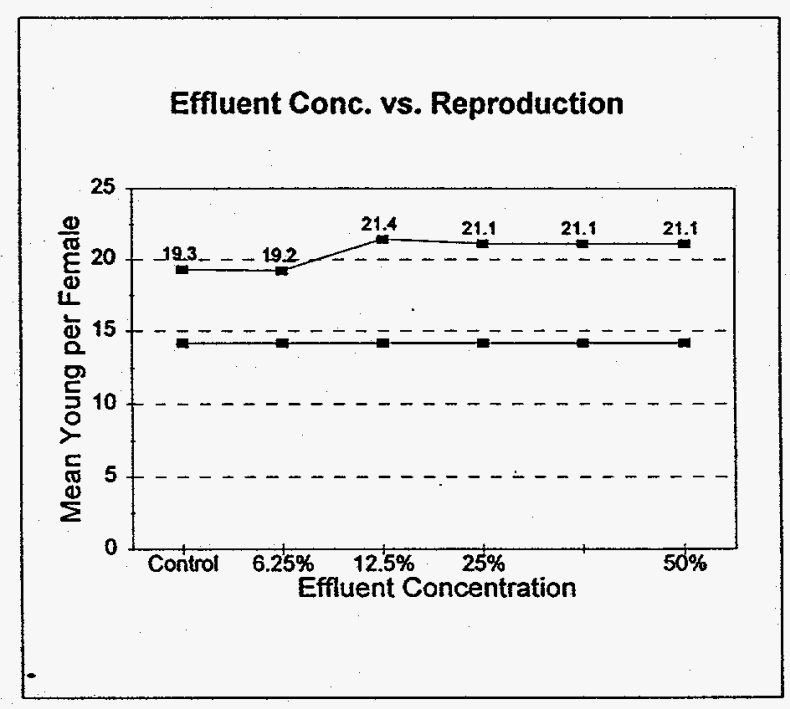

\section{Summary}

The effluent is not chronically toxic to the test organisms. 


\section{CHRONIC DEFINITIVE SURVIVAL AND REPRODUCTION TEST \\ Water Chemistry}

Client: Westinghouse Savannah River Company

Sample Identification: A-006

Test Date: $\quad$ 7/09/96

pH (After Renewal)

\begin{tabular}{|c|c|c|c|c|c|c|c|}
\hline & \multicolumn{7}{|c|}{ Test Concentration } \\
\hline & Control & $6.25 \%$ & $12.5 \%$ & $25 \%$ & $50 \%$ & $100 \%$ & 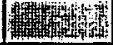 \\
\hline Initial & 6.60 & 7.97 & 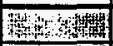 & 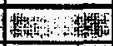 & Antint & 8.38 & 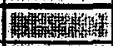 \\
\hline Day 1 & 6.57 & 7.58 & nyty & 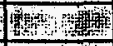 & $y^{4}+4+4$ & 7.82 & - \\
\hline Day 2 & 6.54 & 7.95 & W, & 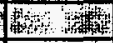 & 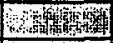 & 7.96 & 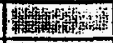 \\
\hline Day 3 & 7.05 & 7.09 & Aक्या & 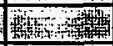 & 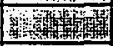 & 7.70 & 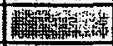 \\
\hline Day 4 & no data & 8.02 & 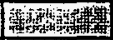 & (1) & 15) & 8.05 & 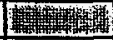 \\
\hline Day 5 & 6.47 & 8.23 & Sta & 1 & 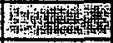 & 8.17 & 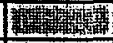 \\
\hline Day 6 & 6.58 & 7.87 & 19.th & 新 & 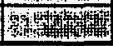 & 7.93 & 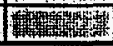 \\
\hline
\end{tabular}

\section{Dissolved Oxygen (After Renewal)}

\begin{tabular}{|c|c|c|c|c|c|c|c|}
\hline & \multicolumn{7}{|c|}{ Test Concentration } \\
\hline & Control & $6.25 \%$ & $12.5 \%$ & $25 \%$ & $50 \%$ & $100 \%$ & \\
\hline Initial & 7.9 & 7.8 & poran & 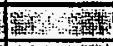 & 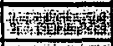 & 8.0 & \\
\hline Day 1 & $\overline{8.2}$ & 7.8 & Hy & 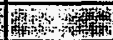 & Wide & 7.9 & \\
\hline Day 2 & 8.5 & 8.1 & 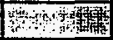 & 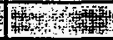 & 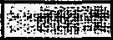 & & \\
\hline Day 3 & 8.1 & 8.0 & - & 4t+4 & The & & \\
\hline Day 4 & no data & no data & thing & 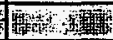 & 194 & & \\
\hline Day 5 & 8.6 & 8.4 & +1 & Hentury & miptory & & \\
\hline Day 6 & 8.5 & 8.5 & Dits & 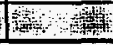 & mith & & \\
\hline
\end{tabular}

\section{Dilution Water \& Effluent Parameters}

\begin{tabular}{|l|r|r|r|r|r|r|}
\hline & Res Cl. & Alkalin & Hard. & Cond. & & \\
\hline & $\mathrm{mg} / \mathrm{L}$ & $\mathrm{mg} / \mathrm{L}$ & $\mathrm{mg} / \mathrm{L}$ & $\mu \mathrm{mhos}$ & & \\
\hline Eff. Sample 1 & 0.02 & 18.5 & 4.0 & 84 & & \\
\hline Eff. Sample 2 & 0.07 & 45.3 & 4.0 & 98 & & \\
\hline Eff. Sample 3 & 0.04 & 45.3 & 8.0 & 96 & & \\
\hline & & & & & & \\
\hline & & & & & & \\
\hline & & & & & & \\
\hline & & & & & & \\
\hline
\end{tabular}

pH (Before Renewal)

\begin{tabular}{|c|c|c|c|c|c|c|}
\hline & \multicolumn{6}{|c|}{ Test Concentration } \\
\hline & Control & $6.25 \%$ & $12.5 \%$ & $25 \%$ & $50 \%$ & $100 \%$ \\
\hline Day 1 & 7.29 & 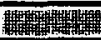 & 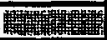 & 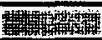 & 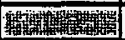 & 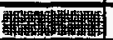 \\
\hline Day 2 & 8.01 & 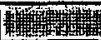 & 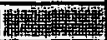 & 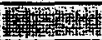 & 4) & 19) \\
\hline Day 3 & $\overline{7.92}$ & 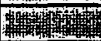 & 10) & 整(t) & 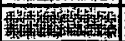 & 特) \\
\hline Day 4 & no data & (1) & (1) & 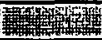 & 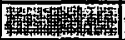 & (9) \\
\hline Day 5 & 8.15 & 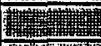 & 1) & 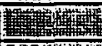 & 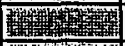 & 19) \\
\hline Day 6 & 8.07 & 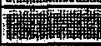 & 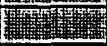 & The & 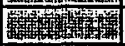 & (5) \\
\hline Final & 8.18 & Tyty & (1) & Thy & 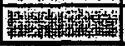 & Tht \\
\hline
\end{tabular}

Dissolved Oxygen (Before Renewal)

\begin{tabular}{|c|c|c|c|c|c|c|}
\hline & \multicolumn{6}{|c|}{ Test Concentration } \\
\hline & Control & $6.25 \%$ & $12.5 \%$ & $25 \%$ & $50 \%$ & $100 \%$ \\
\hline Day 1 & 8.1 & 8.2 & 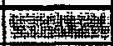 & yisting & absy & 8.4 \\
\hline Day 2 & 8.2 & 8.3 & 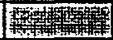 & 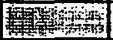 & 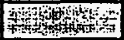 & 8.3 \\
\hline Day 3 & 8.3 & 8.2 & 4 & wis & wry & 8.3 \\
\hline Day 4 & no data & 8.3 & 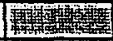 & - & yngy & 8.4 \\
\hline Day 5 & 8.4 & 8.5 & 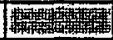 & 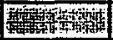 & 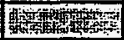 & 8.4 \\
\hline Day 6 & 8.4 & 8.3 & 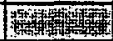 & tonth & 新 & 8.3 \\
\hline Final & & 8.2 & 1) & 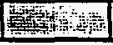 & tots & 8.3 \\
\hline
\end{tabular}

Temperature
\begin{tabular}{|l|r|}
\hline Incubator ${ }^{\circ} \mathrm{C}$ \\
\hline Initial & 24.8 \\
\hline Day 1 & 24.0 \\
\hline Day 2 & 24.0 \\
\hline Day 3 & 25.1 \\
\hline Day 4 & 25.2 \\
\hline Day 5 & 24.0 \\
\hline Day 6 & 24.8 \\
\hline Final & 24.3 \\
\hline
\end{tabular}




\section{Day Chronic Definitive Survival and Reproduction Bioassay \\ Method: EPA/600/4-89/001}

Test Organism:

Ceriodaphnia dubia

Facility:Westinghouse Savannah River Company

Sample ID:Outfall A-006

Date: $\quad 7 / 23 / 96$ 


\section{CHRONIC DEFINITIVE SURVIVAL AND REPRODUCTION TEST \\ Survival and Reproduction Results}

Client: Westinghouse Savannah River Company

Sample Identification: Outfall A-006

Test Date: $\quad 7 / 23 / 96 \quad$ T6124

\begin{tabular}{|c|c|c|c|c|c|c|c|}
\hline \multicolumn{3}{|c|}{ Reproduction at } & \multicolumn{6}{|c|}{ Concentration } \\
\hline Rep. & Day 2/3 & Day 4 & Day 5 & Day 6 & Day 7 & Day 8 & Total \\
\hline 1 & 0 & 4 & 8 & 0 & 13 & 0 & 25 \\
\hline 2 & 0 & 0 & 4 & 7 & 2 & 8 & 21 \\
\hline 3 & 0 & 3 & 5 & 1 & 13 & 0 & 22 \\
\hline 4 & 0 & 4 & 3 & 0 & 13 & 0 & 20 \\
\hline 5 & 0 & 3 & 4 & 8 & 0 & 12 & 27 \\
\hline 6 & 0 & 0 & 4 & 8 & 0 & 1 & 13 \\
\hline 7 & 0 & 0 & 7 & 8 & 11 & 0 & 26 \\
\hline 8 & 0 & 4 & 7 & 0 & 12 & 0 & 23 \\
\hline 9 & 0 & 5 & 6 & 8 & 0 & 0 & 19 \\
\hline 10 & 0 & 0 & 4 & 6 & 0 & 8 & 18 \\
\hline
\end{tabular}

\begin{tabular}{|c|c|c|c|c|c|c|c|}
\hline \multicolumn{9}{|c|}{ Reproduction at } \\
\hline Rep. & Day 2/3 & Day 4 & Day 5 & Day 6 & Day 7 & Day 8 & Total \\
\hline 1 & 0 & 0 & 7 & 8 & 0 & 14 & 29 \\
\hline 2 & 0 & 1 & 3 & 6 & 1 & 11 & 22 \\
\hline 3 & 0 & 0 & 8 & 0 & 13 & 0 & 21 \\
\hline 4 & 0 & 0 & 2 & 9 & 1 & 8 & 20 \\
\hline 5 & 0 & 4 & 8 & 5 & 1 & 0 & 18 \\
\hline 6 & 0 & 0 & 4 & 5 & 0 & 9 & 18 \\
\hline 7 & 0 & 3 & 6 & 9 & 11 & 0 & 29 \\
\hline 8 & 0 & 5 & 5 & 0 & 13 & 0 & 23 \\
\hline 9 & 0 & 2 & 7 & 2 & 0 & 14 & 25 \\
\hline 10 & 0 & 0 & 0 & 2 & 6 & 5 & 13 \\
\hline
\end{tabular}

\begin{tabular}{|c|c|c|c|c|c|c|c|}
\hline \multicolumn{1}{|c|}{ Reproduction at } & $12.5 \%$ & \multicolumn{3}{c|}{ Concentration } \\
\hline Rep. & Day 2/3 & Day 4 & Day 5 & Day 6 & Day 7 & Day 8 & Total \\
\hline 1 & 0 & 4 & 7 & 9 & 0 & 0 & 20 \\
\hline 2 & 0 & 0 & 5 & 9 & 0 & 9 & 23 \\
\hline 3 & 0 & 4 & 7 & 0 & 0 & 10 & 21 \\
\hline 4 & 0 & 3 & 4 & 0 & 3 & 11 & 21 \\
\hline 5 & 0 & 4 & 6 & 10 & 0 & 0 & 20 \\
\hline 6 & 0 & 1 & 2 & 5 & 0 & 4 & 12 \\
\hline 7 & 4 & 0 & 6 & 6 & 0 & 0 & 16 \\
\hline 8 & 0 & 3 & 3 & 10 & 0 & 0 & 16 \\
\hline 9 & 0 & 1 & 6 & 12 & 0 & 9 & 28 \\
\hline 10 & 0 & 0 & 0 & 0 & 4 & 11 & 15 \\
\hline
\end{tabular}

\begin{tabular}{|c|c|c|c|c|c|c|c|}
\hline \multicolumn{2}{|c|}{ Reproduction at } & $25.0 \%$ & \multicolumn{3}{c|}{ Concentration } \\
\hline Rep. & Day 2/3 & Day 4 & Day 5 & Day 6 & Day 7 & Day 8 & Total \\
\hline 1 & 0 & 4 & 5 & 7 & 0 & 10 & 26 \\
\hline 2 & 3 & 0 & 1 & 3 & 0 & 6 & 13 \\
\hline 3 & 0 & 0 & 6 & 0 & 13 & 12 & 31 \\
\hline 4 & 0 & 0 & 5 & 0 & 12 & 0 & 17 \\
\hline 5 & 0 & 3 & 5 & 9 & 0 & 9 & 26 \\
\hline 6 & 0 & 0 & 2 & 3 & 0 & 8 & 13 \\
\hline 7 & 3 & 0 & 0 & 0 & 0 & 0 & 3 \\
\hline 8 & 0 & 4 & 9 & 0 & 0 & 11 & 24 \\
\hline 9 & 0 & 0 & 8 & 3 & 0 & 11 & 22 \\
\hline 10 & 0 & 0 & 7 & 0 & 9 & 6 & 22 \\
\hline
\end{tabular}

\begin{tabular}{|c|c|c|c|c|c|c|c|}
\hline \multicolumn{2}{|c|}{ Reproduction at } & \multicolumn{7}{|c|}{ Concentration } \\
\hline Rep. & Day 2/3 & Day 4 & Day 5 & Day 6 & Day 7 & Day 8 & Total \\
\hline 1 & 0 & 2 & 5 & 8 & 0 & 10 & 25 \\
\hline 2 & 0 & 0 & 6 & 6 & 7 & 0 & 19 \\
\hline 3 & 0 & 1 & 0 & 6 & 10 & 0 & 17 \\
\hline 4 & 0 & 0 & 8 & 5 & 0 & 8 & 21 \\
\hline 5 & 0 & 2 & 0 & 0 & 0 & 6 & 8 \\
\hline 6 & 0 & 0 & 0 & 0 & 0 & 0 & 0 \\
\hline 7 & 0 & 0 & 0 & 0 & 0 & 1 & 1 \\
\hline 8 & 0 & 3 & 6 & 3 & 0 & 8 & 20 \\
\hline 9 & 0 & 4 & 5 & 0 & 0 & 5 & 14 \\
\hline 10 & 0 & 3 & 4 & 0 & 0 & 9 & 16 \\
\hline
\end{tabular}

\begin{tabular}{|c|c|c|c|c|c|c|c|}
\hline \multicolumn{9}{|c|}{ Reproduction at } & $100.0 \%$ & \multicolumn{5}{c|}{ Concentration } \\
\hline Rep. & Day 2/3 & Day 4 & Day 5 & Day 6 & Day 7 & Day 8 & Total \\
\hline 1 & 0 & 3 & 4 & 8 & 1 & 13 & 29 \\
\hline 2 & 6 & 0 & 5 & 0 & 9 & 0 & 20 \\
\hline 3 & 0 & 4 & 6 & 5 & 0 & 8 & 23 \\
\hline 4 & 0 & 0 & 0 & 0 & 0 & 5 & 5 \\
\hline 5 & 3 & 0 & 6 & 7 & 1 & 0 & 17 \\
\hline 6 & 0 & 0 & 6 & 0 & 6 & 3 & 15 \\
\hline 7 & 3 & 0 & 7 & 0 & 0 & 0 & 10 \\
\hline 8 & 0 & 0 & 8 & 6 & 0 & 15 & 29 \\
\hline 9 & 0 & 3 & 7 & 9 & 0 & 0 & 19 \\
\hline 10 & 0 & 3 & 6 & 8 & 0 & 0 & 17 \\
\hline
\end{tabular}

\footnotetext{
$X=$ Dead

$\mathrm{L}=$ Lost or caught in surface tension of water
} 


\section{CHRONIC DEFINITIVE SURVIVAL AND REPRODUCTION TEST \\ Statistical Analyses}

Client: Westinghouse Savannah River Company

Sample Identification: Outfall A-006

Test Date: $\quad$ 7/23/96

\begin{tabular}{|ccr|}
\hline Test for Normality & & \\
Test Used: Shapiro Wilks & $\mathrm{W}=$ & 0.958 \\
& critical & 0.93 \\
The data are normal in distribution & \\
\end{tabular}

\begin{tabular}{|c|c|c|}
\hline \multicolumn{3}{|c|}{ Test for Homogeneity of Variance } \\
\hline Test Used: Bartlett's Test & $\begin{array}{r}\mathrm{B}= \\
\text { critical }\end{array}$ & $\begin{array}{l}8.51 \\
15.1\end{array}$ \\
\hline \multicolumn{3}{|c|}{ The data are homogeneous in variance } \\
\hline
\end{tabular}

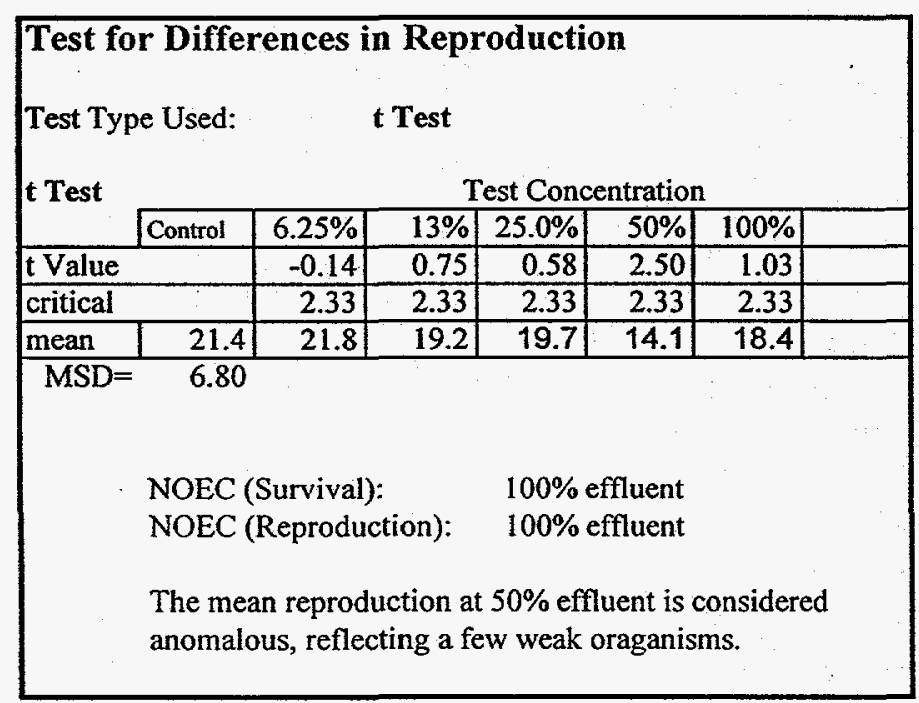

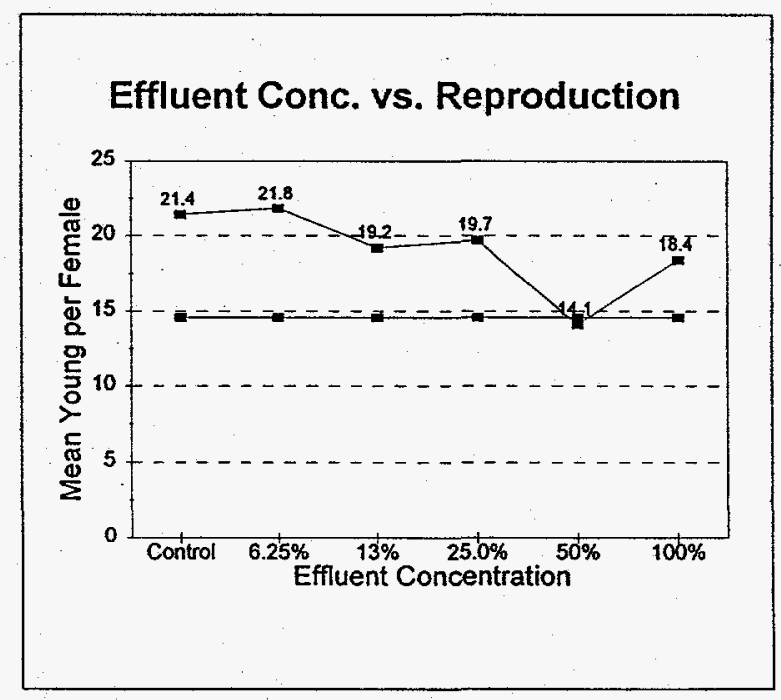

Summary

The effluent is not chronically toxic. 


\section{CHRONIC DEFINITIVE SURVIVAL AND REPRODUCTION TEST \\ Water Chemistry}

Client: Westinghouse Savannah River Company

Sample Identification: Outfall A-006

Test Date: $\quad 7 / 23 / 96$

\section{pH (After Renewal)}

\begin{tabular}{|c|c|c|c|c|c|c|c|}
\hline & \multicolumn{7}{|c|}{ Test Concentration } \\
\hline & Control & $6.25 \%$ & $13 \%$ & $25.0 \%$ & $50 \%$ & $100 \%$ & 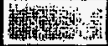 \\
\hline Initial & 6.52 & 7.63 & 14th & Ahy & Bty & 7.43 & 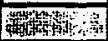 \\
\hline Day 1 & 6.58 & 7.25 & 64 & $\log _{7+1}$ & 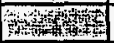 & 7.80 & 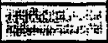 \\
\hline Day 2 & 6.55 & 6.82 & 3ing & 整 & Afd & 7.95 & 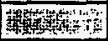 \\
\hline Day 3 & 6.53 & 7.47 & 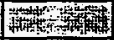 & 4 & 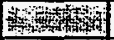 & 8.08 & 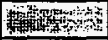 \\
\hline Day 4 & 6.50 & & 51 & 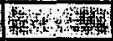 & कy & & Myturat \\
\hline Day 5 & 6.57 & 6.25 & कus & 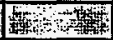 & $\rightarrow n$ ind & 7.97 & 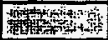 \\
\hline Day 6 & 6.54 & 7.60 & 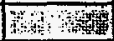 & 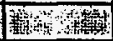 & entry & 7.89 & 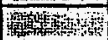 \\
\hline
\end{tabular}

Dissolved Oxygen (After Renewal)

\begin{tabular}{|c|c|c|c|c|c|c|c|}
\hline & \multicolumn{7}{|c|}{ Test Concentration } \\
\hline & Control & $6.25 \%$ & $13 \%$ & $25.0 \%$ & $50 \%$ & $100 \%$ & 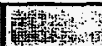 \\
\hline Initial & 8.9 & 8.8 & & & & 9.0 & 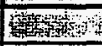 \\
\hline Day 1 & 8.1 & 8.1 & & & & 8.2 & 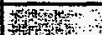 \\
\hline Day 2 & 8.3 & 8.2 & & & & 8.5 & $r_{1}$ \\
\hline Day 3 & $\overline{8.3}$ & $\overline{8.2}$ & & & & 8.4 & The \\
\hline Day 4 & & & & & & & 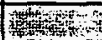 \\
\hline Day 5 & 8.5 & 8.3 & & & & 8.6 & Fite \\
\hline Day 6 & $\overline{8.4}$ & $\overline{8.3}$ & & & & 8.4 & 19tis \\
\hline
\end{tabular}

\section{Dilution Water \& Effluent Parameters}

\begin{tabular}{|c|c|c|c|c|c|c|}
\hline & Res CI. & Alkalin & Hard. & Cond. & tom & 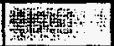 \\
\hline & $\mathrm{mg} / \mathrm{L}$ & $\mathrm{mg} / \mathrm{L}$ & $\mathrm{mg} / \mathrm{L}$ & $\mu \mathrm{mhos}$ & ry & Men \\
\hline Eff. Sample 1 & 0.03 & 41.2 & 6 & 95.7 & Why & 政W \\
\hline Eff. Sample 2 & 0.06 & 45.3 & 4 & 98.1 & 6 & Wha \\
\hline \multirow[t]{2}{*}{ Eff. Sample 3} & 0.05 & 48.3 & 8 & 101.2 & WHe & wit \\
\hline & & & & & Wh & 80 \\
\hline \multirow[t]{3}{*}{ Dilution Water } & & 4.1 & 4 & 15 & की & ast \\
\hline & & & & & ntort & W \\
\hline & & & & & $20 \%$ & tes: \\
\hline
\end{tabular}

\section{$\mathrm{pH}$ (Before Renewal)}

\begin{tabular}{|c|c|c|c|c|c|c|c|}
\hline & \multicolumn{7}{|c|}{ Test Concentration } \\
\hline & Control & $6.25 \%$ & $13 \%$ & $25.0 \%$ & $50 \%$ & $100 \%$ & 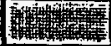 \\
\hline Day 1 & 7.25 & 6.82 & 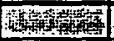 & Hathos & whom & 7.81 & (1) \\
\hline Day 2 & 7.89 & 7.63 & 1) & 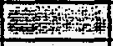 & 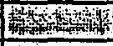 & 7.72 & 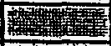 \\
\hline Day 3 & 7.71 & 7.36 & 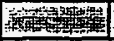 & 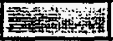 & mon & 7.99 & 174 \\
\hline Day 4 & & & 整 & 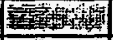 & 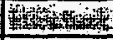 & & P \\
\hline Day 5 & 6.53 & 6.36 & 10 & 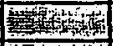 & 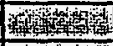 & 7.03 & 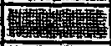 \\
\hline Day 6 & 7.88 & 7.72 & W & 30 & 44 & 7.82 & 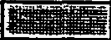 \\
\hline Final & 7.93 & 7.79 & 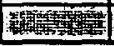 & 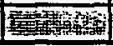 & 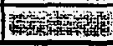 & 8.26 & at \\
\hline
\end{tabular}

\section{Dissolved Oxygen (Before Renewal)}

\begin{tabular}{|c|c|c|c|c|c|c|c|}
\hline & \multicolumn{7}{|c|}{ Test Concentration } \\
\hline & Control & $6.25 \%$ & $13 \%$ & $25.0 \%$ & $50 \%$ & $100 \%$ & (2) \\
\hline Day 1 & 8.1 & 8.1 & 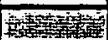 & 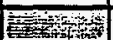 & 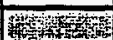 & 8.1 & 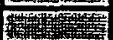 \\
\hline Day 2 & 8.2 & 8.4 & Sitiving & 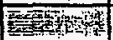 & (4t) & $\overline{8.2}$ & W \\
\hline Day 3 & 8.3 & 8.3 & 3ats & W. & hision & 8.4 & \\
\hline Day 4 & & & 1 & 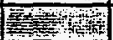 & Wringush & & A \\
\hline Day 5 & 8.4 & 8.3 & 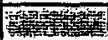 & 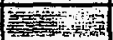 & 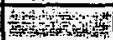 & 8.4 & 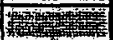 \\
\hline Day 6 & 8.2 & 8.2 & 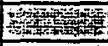 & 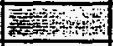 & mats & 8.3 & 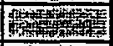 \\
\hline Final & 8.3 & 8.1 & 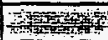 & mans & 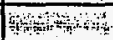 & 8.2 & 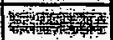 \\
\hline
\end{tabular}

Temperature
\begin{tabular}{|l|r|}
\hline \multicolumn{2}{|c|}{ Incubator ${ }^{\circ} \mathrm{C}$} \\
\hline Initial & 25.5 \\
\hline Day 1 & 24.4 \\
\hline Day 2 & 24.3 \\
\hline Day 3 & 24.8 \\
\hline Day 4 & 24.5 \\
\hline Day 5 & 24.5 \\
\hline Day 6 & 25.3 \\
\hline Final & 24.7 \\
\hline
\end{tabular}




\section{Day Chronic Definitive Survival and Reproduction Bioassay}

Method: EPA/600/4-89/001

Test Organism:

Ceriodaphnia dubia

Facility: Westinghouse Savannah River Company

Sample ID:T6098 (AT-001)

Date: $\quad 7-16-96$ 


\title{
CHRONIC DEFINITIVE SURVIVAL AND REPRODUCTION TEST Survival and Reproduction Results
}

Client: Westinghouse Savannah River Company

Sample Identification: T6098 (AT-001)

Test Date: $\quad$ 7-16-96

\begin{tabular}{|c|c|c|c|c|c|c|c|}
\hline \multicolumn{1}{|c|}{ Reproduction at } \\
\hline Rep. & Day 2/3 & Day 4 & Day 5 & Day 6 & Day 7 & Day 8 & Total \\
\hline 1 & 0 & 4 & 8 & 0 & 10 & 0 & 22 \\
\hline 2 & 0 & 2 & 7 & 0 & 0 & 8 & 17 \\
\hline 3 & 0 & 5 & 7 & 0 & 11 & 0 & 23 \\
\hline 4 & 0 & $\mathrm{~L}$ & $\mathrm{~L}$ & $\mathrm{~L}$ & $\mathrm{~L}$ & $\mathrm{~L}$ & $\mathrm{~L}$ \\
\hline 5 & 0 & 3 & 10 & 0 & 0 & 0 & 13 \\
\hline 6 & 0 & 6 & 8 & 0 & 11 & 0 & 25 \\
\hline 7 & $\mathrm{~L}$ & $\mathrm{~L}$ & $\mathrm{~L}$ & $\mathrm{~L}$ & $\mathrm{~L}$ & $\mathrm{~L}$ & $\mathrm{~L}$ \\
\hline 8 & 0 & 0 & 5 & 10 & 0 & 1 & 16 \\
\hline 9 & 0 & 0 & 6 & 10 & 0 & 6 & 22 \\
\hline 10 & 0 & 4 & 7 & 0 & 12 & 0 & 23 \\
\hline
\end{tabular}

\begin{tabular}{|c|c|c|c|c|c|c|c|}
\hline \multicolumn{2}{|c|}{ Reproduction at } & $6.0 \%$ & Concentration \\
\hline Rep. & Day 2/3 & Day 4 & Day 5 & Day 6 & Day 7 & Day 8 & Total \\
\hline 1 & 0 & 3 & 8 & 0 & 0 & 3 & 14 \\
\hline 2 & 0 & 3 & 7 & 0 & 10 & 0 & 20 \\
\hline 3 & 0 & 4 & 8 & 9 & 0 & 0 & 21 \\
\hline 4 & 0 & 0 & 12 & 9 & 0 & 0 & 21 \\
\hline 5 & 0 & 6 & 9 & 0 & 0 & 3 & 18 \\
\hline 6 & 0 & 5 & 9 & 11 & 0 & 0 & 25 \\
\hline 7 & 0 & 4 & 6 & 0 & 8 & 0 & 18 \\
\hline 8 & L & L & L & L & L & L & L \\
\hline 9 & 0 & 5 & 4 & 0 & 13 & 0 & 22 \\
\hline 10 & 0 & 3 & 9 & 0 & 14 & 0 & 26 \\
\hline
\end{tabular}

\begin{tabular}{|c|c|c|c|c|c|c|c|}
\hline \multicolumn{3}{|c|}{ Reproduction at } & $12.0 \%$ & Concentration \\
\hline Rep. & Day 2/3 & Day 4 & Day 5 & Day 6 & Day 7 & Day 8 & Total \\
\hline 1 & 0 & 0 & 8 & 0 & 0 & 1 & 9 \\
\hline 2 & 0 & 0 & 4 & $L$ & $L$ & $L$ & L \\
\hline 3 & 0 & 0 & 7 & 4 & 5 & 5 & 21 \\
\hline 4 & 4 & 0 & 8 & 9 & 0 & 0 & 21 \\
\hline 5 & 0 & 4 & 7 & 0 & 12 & 0 & 23 \\
\hline 6 & 0 & 5 & 8 & 0 & 0 & 0 & 13 \\
\hline 7 & 0 & 5 & 9 & 0 & 3 & 12 & 29 \\
\hline 8 & 0 & 5 & 7 & 0 & 9 & 0 & 21 \\
\hline 9 & 0 & 0 & 8 & 11 & 0 & 0 & 19 \\
\hline 10 & 0 & 4 & 7 & 0 & 11 & 0 & 22 \\
\hline
\end{tabular}

\begin{tabular}{|c|c|c|c|c|c|c|c|}
\hline \multicolumn{4}{|c|}{ Reproduction at } & $25.0 \%$ & \multicolumn{1}{c|}{ Concentration } \\
\hline Rep. & Day 2/3 & Day 4 & Day 5 & Day 6 & Day 7 & Day 8 & Total \\
\hline 1 & 0 & 2 & 6 & 0 & 13 & 0 & 21 \\
\hline 2 & 0 & 0 & 3 & 5 & 9 & 0 & 17 \\
\hline 3 & 0 & 0 & 8 & 0 & 8 & 0 & 16 \\
\hline 4 & 0 & 3 & 7 & 10 & 0 & 0 & 20 \\
\hline 5 & 0 & 5 & 9 & 0 & 13 & 0 & 27 \\
\hline 6 & $\mathrm{~L}$ & $\mathrm{~L}$ & $\mathrm{~L}$ & $\mathrm{~L}$ & $\mathrm{~L}$ & $\mathrm{~L}$ & $\mathrm{~L}$ \\
\hline 7 & 0 & 4 & 9 & 0 & 8 & 0 & 21 \\
\hline 8 & 0 & 3 & 7 & 0 & 10 & 0 & 20 \\
\hline 9 & 0 & 5 & 7 & 11 & 0 & 0 & 23 \\
\hline 10 & 0 & 2 & 5 & 0 & 12 & 0 & 19 \\
\hline
\end{tabular}

\begin{tabular}{|c|l|l|l|l|l|l|c|}
\hline \multicolumn{6}{|l|}{ Reproduction at } & \multicolumn{6}{l|}{ Concentration } \\
\hline Rep. & Day $2 / 3$ & Day 4 & Day 5 & Day 6 & Day 7 & Day 8 & Total \\
\hline 1 & $\mathrm{X}$ & $\mathrm{X}$ & $\mathrm{X}$ & $\mathrm{X}$ & $\mathrm{X}$ & $\mathrm{X}$ & 0 \\
\hline 2 & $\mathrm{X}$ & $\mathrm{X}$ & $\mathrm{X}$ & $\mathrm{X}$ & $\mathrm{X}$ & $\mathrm{X}$ & 0 \\
\hline 3 & $\mathrm{X}$ & $\mathrm{X}$ & $\mathrm{X}$ & $\mathrm{X}$ & $\mathrm{X}$ & $\mathrm{X}$ & 0 \\
\hline 4 & $\mathrm{X}$ & $\mathrm{X}$ & $\mathrm{X}$ & $\mathrm{X}$ & $\mathrm{X}$ & $\mathrm{X}$ & 0 \\
\hline 5 & $\mathrm{X}$ & $\mathrm{X}$ & $\mathrm{X}$ & $\mathrm{X}$ & $\mathrm{X}$ & $\mathrm{X}$ & 0 \\
\hline 6 & $\mathrm{X}$ & $\mathrm{X}$ & $\mathrm{X}$ & $\mathrm{X}$ & $\mathrm{X}$ & $\mathrm{X}$ & 0 \\
\hline 7 & $\mathrm{X}$ & $\mathrm{X}$ & $\mathrm{X}$ & $\mathrm{X}$ & $\mathrm{X}$ & $\mathrm{X}$ & 0 \\
\hline 8 & $\mathrm{X}$ & $\mathrm{X}$ & $\mathrm{X}$ & $\mathrm{X}$ & $\mathrm{X}$ & $\mathrm{X}$ & 0 \\
\hline 9 & $\mathrm{X}$ & $\mathrm{X}$ & $\mathrm{X}$ & $\mathrm{X}$ & $\mathrm{X}$ & $\mathrm{X}$ & 0 \\
\hline 10 & $\mathrm{X}$ & $\mathrm{X}$ & $\mathrm{X}$ & $\mathrm{X}$ & $\mathrm{X}$ & $\mathrm{X}$ & 0 \\
\hline
\end{tabular}

\begin{tabular}{|c|c|c|c|c|c|c|c|}
\hline \multicolumn{3}{|c|}{ Reproduction at } & \multicolumn{5}{|c|}{$100.0 \%$ Concentration } \\
\hline Rep. & Day $2 / 3$ & Day 4 & Day 5 & Day 6 & Day 7 & Day 8 & Total \\
\hline 1 & $\bar{X}$ & $x$ & $x$ & $\bar{X}$ & $x$ & $\mathrm{X}$ & 0 \\
\hline 2 & $\bar{x}$ & $\bar{x}$ & $\bar{x}$ & $\bar{x}$ & $\bar{x}$ & $\bar{x}$ & 0 \\
\hline 3 & $\bar{x}$ & $\bar{X}$ & $\bar{X}$ & $\bar{X}$ & $\bar{x}$ & $\bar{x}$ & $\overline{0}$ \\
\hline 4 & $\bar{x}$ & $\bar{x}$ & $\bar{x}$ & $\bar{x}$ & $x$ & $\bar{x}$ & 0 \\
\hline 5 & $\bar{X}$ & $\bar{X}$ & $\bar{x}$ & $\mathrm{X}$ & $\bar{x}$ & $\bar{x}$ & 0 \\
\hline 6 & $\mathrm{X}$ & $\bar{X}$ & $\bar{X}$ & $\mathrm{X}$ & $x$ & $\bar{x}$ & 0 \\
\hline 7 & $\bar{x}$ & $\bar{x}$ & $\bar{x}$ & $\bar{x}$ & $\mathrm{X}$ & $\bar{x}$ & 0 \\
\hline 8 & $\bar{x}$ & 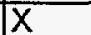 & $x$ & $\bar{X}$ & x & $\bar{x}$ & 0 \\
\hline 9 & $\bar{x}$ & $\bar{x}$ & $\bar{x}$ & $\bar{x}$ & $\bar{x}$ & $x$ & 0 \\
\hline 10 & $x$ & $x$ & $\mathrm{x}$ & $x$ & $\bar{x}$ & $x$ & 0 \\
\hline
\end{tabular}

\author{
$X=$ Dead \\ $L=$ Lost or caught in surface tension of water
}




\section{CHRONIC DEFINITIVE SURVIVAL AND REPRODUCTION TEST \\ Statistical Analyses}

Client: Westinghouse Savannah River Company

Sample Identification: T6098 (AT-001)

Test Date: $\quad$ 7-16-96

\begin{tabular}{|lrr}
\hline Test for Normality & & \\
& & \\
Test Used: Shapiro Wilks & W & 0.971 \\
& critical & 0.919
\end{tabular}

Test for Homogeneity of Variance

Test Used: Bartlett's Test $\quad \mathrm{B}=\quad 3.06$

critical $\quad 13.28$

The data are normal in distribution

The data are homogeneous in variance

Test for Differences in Reproduction

Test Type Used: $\quad$ t Test

t Test Test Concentration

\begin{tabular}{|l|r|r|r|r|r|r|r|}
\hline & \multicolumn{1}{|l|}{ Control } & $6.0 \%$ & $12.0 \%$ & $25.0 \%$ & $50 \%$ & $100 \%$ & \\
\hline t Value & -0.20 & 0.16 & -0.15 & NA & NA & \\
\hline critical & 2.33 & 2.33 & 2.33 & 2.33 & 2.33 & \\
\hline mean & 20.1 & 20.6 & 19.8 & 20.4 & 0.0 & 0.0 & \\
\hline$\%$ mortality & $0 \%$ & $0 \%$ & $0 \%$ & $100 \%$ & $100 \%$ & \\
\hline
\end{tabular}

NOEC (Survival): $\quad 25 \%$ effluent

NOEC (Reproduction): $25 \%$ effluent

Effluent Conc. vs. Reproduction

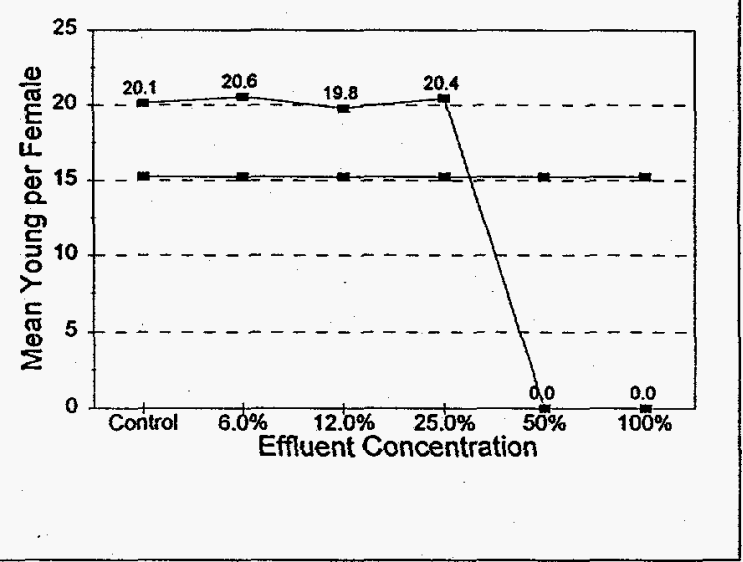

Summary

The effluent is toxic at concentrations of $50 \%$ and higher. 


\section{CHRONIC DEFINITIVE SURVIVAL AND REPRODUCTION TEST \\ Water Chemistry}

Client: Westinghouse Savannah River Company

Sample Identification: T6098 (AT-001)

Test Date: $\quad$ 7-16-96

pH (After Renewal)

\begin{tabular}{|c|c|c|c|c|c|c|c|}
\hline & \multicolumn{7}{|c|}{ Test Concentration } \\
\hline & Control & $6.0 \%$ & $12.0 \%$ & $25.0 \%$ & $50 \%$ & $100 \%$ & \\
\hline Initial & 7.97 & 7.80 & 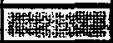 & 14 & 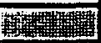 & 7.74 & \\
\hline Day 1 & 得 & 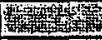 & 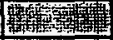 & 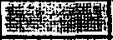 & (17) & 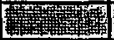 & 1) \\
\hline Day 2 & 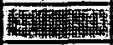 & 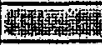 & 15 & 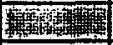 & 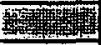 & (1) & 7) \\
\hline Day 3 & Hem & 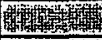 & 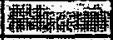 & 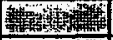 & A M & 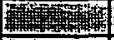 & AIt \\
\hline Day 4 & 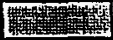 & 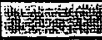 & 74 & 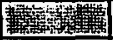 & 整 & (1) & (1) \\
\hline Day 5 & 4 & 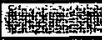 & 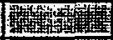 & 1) & 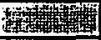 & 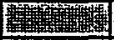 & 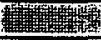 \\
\hline Day 6 & 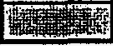 & 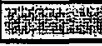 & (1) & 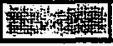 & 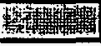 & 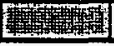 & 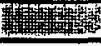 \\
\hline
\end{tabular}

Dissolved Oxygen (After Renewal)

\begin{tabular}{|c|c|c|c|c|c|c|c|}
\hline & \multicolumn{7}{|c|}{ Test Concentration } \\
\hline & Control & $6.0 \%$ & $12.0 \%$ & $25.0 \%$ & $50 \%$ & $100 \%$ & \\
\hline Initial & 8.6 & 8.3 & 10 & 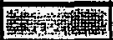 & 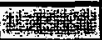 & 8.6 & \\
\hline Day 1 & 8.5 & mythest & 4 & yon & 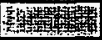 & 物 & 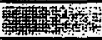 \\
\hline Day 2 & 8.6 & 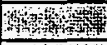 & 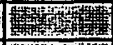 & ring & 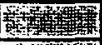 & 19 & 10 \\
\hline Day 3 & 8.7 & 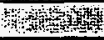 & Hetw & 11 & 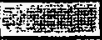 & $=1$ (1) & 4 \\
\hline Day 4 & 8.6 & Hating & 1) & Prot & 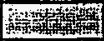 & Handy & 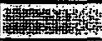 \\
\hline Day 5 & 8.6 & nty & 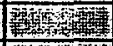 & 4-14 & Find & 整 & 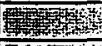 \\
\hline Day 6 & 8.7 & 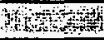 & 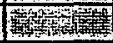 & 14. & 4tist & क & The \\
\hline
\end{tabular}

Dilution Water \& Effluent Parameters

\begin{tabular}{|l|r|r|r|r|r|r|}
\hline & Res Cl. & Alkalin & Hard. & Cond. & & \\
\hline & $\mathrm{mg} / \mathrm{L}$ & $\mathrm{mg} / \mathrm{L}$ & $\mathrm{mg} / \mathrm{L}$ & $\mu \mathrm{mhos}$ & & \\
\hline Eff. Sample 1 & 0.02 & 28.8 & 6.0 & 80 & & \\
\hline Eff. Sample 2 & 0.06 & 25.2 & 10.0 & 79 & & \\
\hline Eff. Sample 3 & N/A & 29.4 & 6.0 & 86 & & \\
\hline & & & & & & \\
\hline Dilution Water & & 2.1 & 4.0 & 30 & & \\
\hline & & & & & & \\
\hline & & & & & & \\
\hline
\end{tabular}

Dilution water was Upper Three Runs Creek water pH adjusted to 6.5.
$\mathrm{pH}$ (Before Renewal)

\begin{tabular}{|c|c|c|c|c|c|c|c|}
\hline & \multicolumn{7}{|c|}{ Test Concentration } \\
\hline & Control & $6.0 \%$ & $12.0 \%$ & $25.0 \%$ & $50 \%$ & $100 \%$ & \\
\hline Day 1 & 8.18 & 7.15 & 4 & 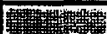 & 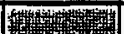 & 7.82 & \\
\hline Day 2 & 8.09 & 8.17 & Wat & W & 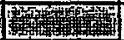 & 7.83 & \\
\hline Day 3 & 8.24 & 7.55 & 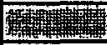 & 4 & 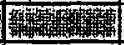 & 7.61 & \\
\hline Day 4 & 8.19 & 7.32 & (T) & 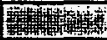 & 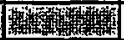 & 7.61 & \\
\hline Day 5 & 7.98 & 7.46 & W & 7.48 & 特 & & \\
\hline Day 6 & 8.17 & 7.43 & 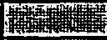 & 7.44 & 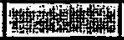 & & \\
\hline Final & 8.19 & 7.14 & 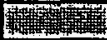 & 7.16 & My & & \\
\hline
\end{tabular}

\section{Dissolved Oxygen (Before Renewal)}

\begin{tabular}{|c|c|c|c|c|c|c|c|}
\hline & \multicolumn{7}{|c|}{ Test Concentration } \\
\hline & Control & $6.0 \%$ & $12.0 \%$ & $25.0 \%$ & $50 \%$ & $100 \%$ & \\
\hline Day 1 & 8.2 & 8.0 & 灌 & 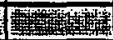 & & 8.3 & \\
\hline Day 2 & 8.5 & 8.1 & 14 & 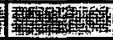 & $4=$ & 8.1 & \\
\hline Day 3 & 8.5 & 8.6 & 15 & 政 & +7 & 8.6 & \\
\hline Day 4 & 8.6 & 8.4 & (1) & $\sqrt{14}$ & III & 8.4 & \\
\hline Day 5 & 8.5 & 8.1 & 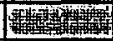 & 8.2 & $\mathrm{Bu}^{2}$ & 8.2 & \\
\hline Day 6 & 8.6 & 8.3 & 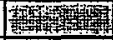 & 8.3 & 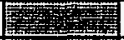 & & \\
\hline Final & 8.6 & 8.3 & 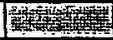 & 8.2 & 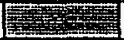 & & \\
\hline
\end{tabular}

\section{Temperature}

\begin{tabular}{|l|r|}
\hline \multicolumn{2}{|c|}{ Incubator ${ }^{\circ} \mathrm{C}$} \\
\hline Initial & 24.2 \\
\hline Day 1 & 24.3 \\
\hline Day 2 & 24.3 \\
\hline Day 3 & 24.4 \\
\hline Day 4 & 25.1 \\
\hline Day 5 & 25.9 \\
\hline Day 6 & 24.5 \\
\hline Final & 24.4 \\
\hline
\end{tabular}




\section{Day Chronic Definitive Survival and Reproduction Bioassay}

Method: EPA/600/4-89/001

Test Organism:

Ceriodaphnia dubia

Facility:Westinghouse Savannah River Company

Sample ID:T6099 (WE-001)

Date: 7-16-96 


\section{CHRONIC DEFINITIVE SURVIVAL AND REPRODUCTION TEST Survival and Reproduction Results}

Client: Westinghouse Savannah River Company

Sample Identification: T6099 (WE-001)

Test Date: $\quad 7-16-96$

\begin{tabular}{|c|c|c|c|c|c|c|c|}
\hline \multicolumn{3}{|c|}{ Reproduction at } & \multirow{2}{*}{$\frac{0.0 \%}{\text { Day } 5}$} & \multicolumn{4}{|c|}{ Concentration } \\
\hline Rep. & Day $2 / 3$ & Day 4 & & Day 6 & Day 7 & Day 8 & Total \\
\hline 1 & 0 & 4 & 8 & 0 & 10 & 0 & 22 \\
\hline 2 & 0 & 2 & 7 & 0 & 0 & 8 & 17 \\
\hline 3 & 0 & 5 & 7 & 0 & 11 & 0 & 23 \\
\hline 4 & 0 & $\mathbf{L}$ & $\mathbf{L}$ & $L$ & L & $L$ & $\mathrm{~L}$ \\
\hline 5 & 0 & 3 & 10 & 0 & 0 & 0 & 13 \\
\hline 6 & 0 & 6 & 8 & 0 & 11 & 0 & 25 \\
\hline 7 & 0 & 5 & L & L & L & $L$ & $L$ \\
\hline 8 & 0 & 0 & 5 & 10 & 0 & 1 & 16 \\
\hline 9 & 0 & 0 & 6 & 10 & 0 & 6 & 22 \\
\hline 10 & 0 & 4 & 7 & 0 & 12 & 0 & 23 \\
\hline
\end{tabular}

\begin{tabular}{|c|c|c|c|c|c|c|c|}
\hline \multicolumn{3}{|c|}{ Reproduction at } & $6.0 \%$ & \multicolumn{1}{c|}{ Concentration } \\
\hline Rep. & Day 2/3 & Day 4 & Day 5 & Day 6 & Day 7 & Day 8 & Total \\
\hline 1 & 0 & 1 & 0 & 11 & 0 & 7 & 19 \\
\hline 2 & 0 & 2 & 11 & 0 & 13 & 0 & 26 \\
\hline 3 & 0 & 5 & 8 & 10 & 0 & 0 & 23 \\
\hline 4 & 0 & 5 & 9 & 7 & 0 & 0 & 21 \\
\hline 5 & 0 & 4 & 0 & 8 & 12 & 0 & 24 \\
\hline 6 & 0 & 0 & 8 & $\mathrm{~L}$ & $\mathrm{~L}$ & $\mathrm{~L}$ & $\mathrm{~L}$ \\
\hline 7 & 0 & 0 & 10 & 11 & 0 & 0 & 21 \\
\hline 8 & 0 & 5 & 11 & 13 & 0 & 0 & 29 \\
\hline 9 & 0 & 0 & 7 & 9 & 0 & 0 & 16 \\
\hline 10 & $\mathrm{~L}$ & $\mathrm{~L}$ & $\mathrm{~L}$ & $\mathrm{~L}$ & $\mathrm{~L}$ & $\mathrm{~L}$ & $\mathrm{~L}$ \\
\hline
\end{tabular}

\begin{tabular}{|c|c|c|c|c|c|c|c|}
\hline \multicolumn{1}{|c|}{ Reproduction at } & $12.0 \%$ & \multicolumn{5}{c|}{ Concentration } \\
\hline Rep. & Day 2/3 & Day 4 & Day 5 & Day 6 & Day 7 & Day 8 & Total \\
\hline 1 & 0 & 3 & 6 & 0 & 12 & 0 & 21 \\
\hline 2 & 0 & 5 & 8 & 0 & 13 & 0 & 26 \\
\hline 3 & 0 & 0 & 4 & 11 & 8 & 0 & 23 \\
\hline 4 & 0 & 7 & 13 & 8 & 0 & 0 & 28 \\
\hline 5 & 0 & $\mathrm{~L}$ & $\mathrm{~L}$ & $\mathrm{~L}$ & $\mathrm{~L}$ & $\mathrm{~L}$ & $\mathrm{~L}$ \\
\hline 6 & 0 & 5 & 9 & 0 & 15 & 0 & 29 \\
\hline 7 & 0 & 4 & 8 & 0 & 13 & 0 & 25 \\
\hline 8 & 0 & 4 & 7 & 13 & 0 & 0 & 24 \\
\hline 9 & 0 & 5 & 8 & 10 & 0 & 0 & 23 \\
\hline 10 & 0 & 0 & $\mathrm{~L}$ & $\mathrm{~L}$ & $\mathrm{~L}$ & $\mathrm{~L}$ & $\mathrm{~L}$ \\
\hline
\end{tabular}

\begin{tabular}{|c|c|c|c|c|c|c|c|}
\hline \multicolumn{2}{|c|}{ Reproduction at } & $25.0 \%$ & \multicolumn{1}{c|}{ Concentration } \\
\hline Rep. & Day 2/3 & Day 4 & Day 5 & Day 6 & Day 7 & Day 8 & Total \\
\hline 1 & 0 & 4 & 8 & 2 & 10 & 0 & 24 \\
\hline 2 & 0 & 2 & 11 & 0 & 9 & 0 & 22 \\
\hline 3 & 0 & 0 & 6 & 10 & 8 & 0 & 24 \\
\hline 4 & 0 & 5 & 10 & 11 & 0 & 0 & 26 \\
\hline 5 & 0 & $L$ & $L$ & $L$ & $L$ & $L$ & L \\
\hline 6 & 0 & 4 & 9 & 0 & 12 & 0 & 25 \\
\hline 7 & 0 & 4 & 6 & 7 & 0 & 0 & 17 \\
\hline 8 & 0 & 6 & 9 & 9 & 0 & 0 & 24 \\
\hline 9 & 0 & 3 & 10 & 10 & 0 & 0 & 23 \\
\hline 10 & 1 & 3 & 7 & 0 & 15 & 0 & 26 \\
\hline
\end{tabular}

\begin{tabular}{|c|c|c|c|c|c|c|c|}
\hline \multicolumn{2}{|c|}{ Reproduction at } & \multicolumn{7}{c|}{$5 \%$ Concentration } \\
\hline Rep. & Day 2/3 & Day 4 & Day 5 & Day 6 & Day 7 & Day 8 & Total \\
\hline 1 & 0 & 3 & 10 & 11 & 0 & 0 & 24 \\
\hline 2 & 0 & 1 & 9 & 13 & 0 & 0 & 23 \\
\hline 3 & 0 & 3 & 6 & 12 & 0 & 0 & 21 \\
\hline 4 & 0 & 5 & 11 & 11 & 0 & 0 & 27 \\
\hline 5 & 0 & 2 & 12 & 0 & 16 & 0 & 30 \\
\hline 6 & 0 & 4 & 6 & 11 & 0 & 0 & 21 \\
\hline 7 & 0 & 3 & 9 & 13 & 0 & 0 & 25 \\
\hline 8 & 0 & 0 & 10 & 0 & 14 & 0 & 24 \\
\hline 9 & 0 & 5 & 10 & 8 & 0 & 0 & 23 \\
\hline 10 & 0 & 4 & 6 & 0 & 5 & 0 & 15 \\
\hline
\end{tabular}

\begin{tabular}{|c|c|c|c|c|c|c|c|}
\hline \multicolumn{3}{|c|}{ Reproduction at } & \multicolumn{5}{|c|}{$100.0 \%$ Concentration } \\
\hline Rep. & Day $2 / 3$ & Day 4 & Day 5 & Day 6 & Day 7 & Day 8 & Total \\
\hline 1 & 0 & 4 & 7 & 14 & 0 & 0 & 25 \\
\hline 2 & 0 & 7 & 8 & 13 & 0 & 0 & 28 \\
\hline 3 & 0 & 4 & 8 & 11 & 0 & 0 & 23 \\
\hline 4 & 0 & 5 & 7 & 11 & 0 & 0 & 23 \\
\hline 5 & 0 & 5 & 11 & 10 & 0 & 0 & 26 \\
\hline 6 & 0 & 5 & 10 & 10 & 0 & 0 & 25 \\
\hline 7 & 0 & 4 & 9 & 12 & 0 & 0 & 25 \\
\hline 8 & 0 & 1 & 10 & 18 & 0 & 0 & 29 \\
\hline 9 & 0 & 4 & 8 & 10 & 0 & 0 & 22 \\
\hline 10 & 0 & 3 & 5 & 14 & 0 & 0 & 22 \\
\hline
\end{tabular}

$X=$ Dead

$L=$ Lost or caught in surface tension of water 


\section{CHRONIC DEFINITIVE SURVIVAL AND REPRODUCTION TEST \\ Statistical Analyses}

Client: Westinghouse Savannah River Company

Sample Identification: T6099 (WE-001)

Test Date: $\quad$ 7-16-96

\begin{tabular}{|ccc|}
\hline Test for Normality & & \\
Test Used: Shapiro Wilks & W= & 0.979 \\
& critical & 0.93 \\
The data are normal in distribution & \\
& \\
\end{tabular}

\begin{tabular}{|lrr|}
\hline \multicolumn{2}{|c|}{ Test for Homogeneity of Variance } \\
Test Used: Bartlett's Test & $\mathrm{B}=$ & 5.50 \\
critical & 15.1 \\
The data are homogeneous in variance & \\
\end{tabular}

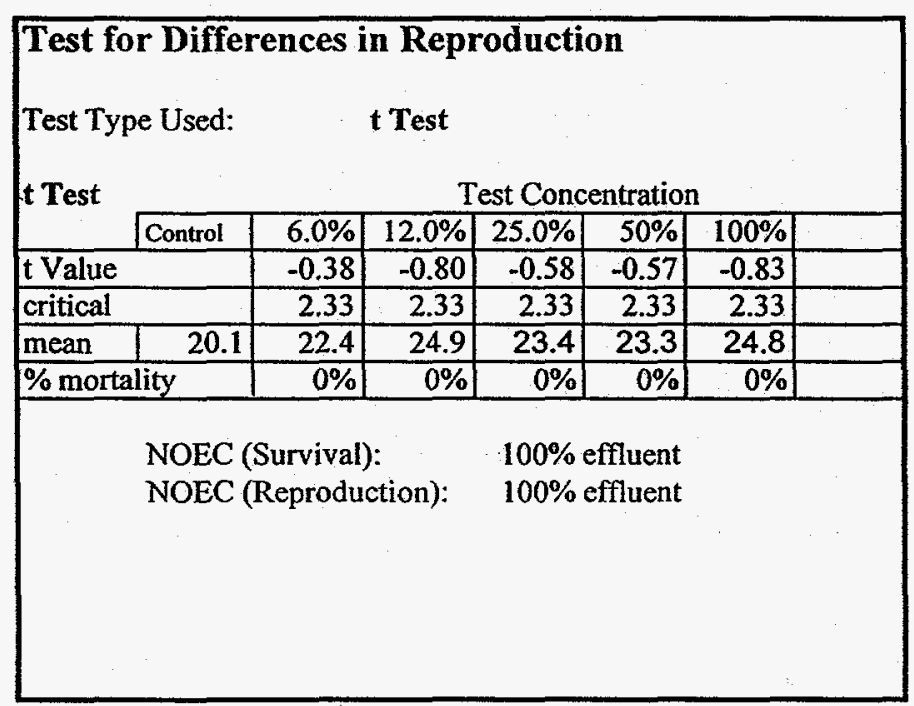

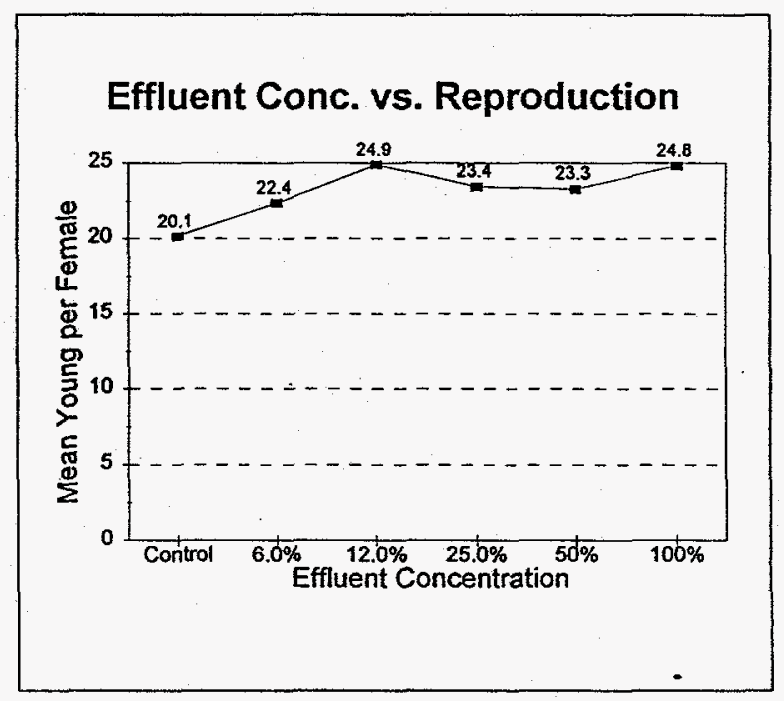

Summary
The sample is not toxic.




\section{CHRONIC DEFINITIVE SURVIVAL AND REPRODUCTION TEST \\ Water Chemistry}

Client: Westinghouse Savannah River Company

Sample Identification: T6099 (WE-001)

Test Date: $\quad 7-16-96$

\section{pH (After Renewal)}

\begin{tabular}{|c|c|c|c|c|c|c|c|}
\hline & \multicolumn{7}{|c|}{ Test Concentration } \\
\hline & Control & $6.0 \%$ & $12.0 \%$ & $25.0 \%$ & $50 \%$ & $100 \%$ & \\
\hline Initial & 7.97 & 7.80 & 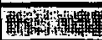 & 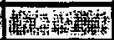 & 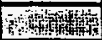 & 7.74 & \\
\hline Day 1 & 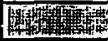 & (1) & 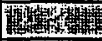 & 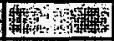 & 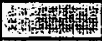 & 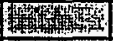 & mist \\
\hline Day 2 & 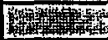 & 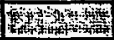 & 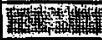 & 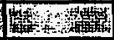 & 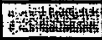 & 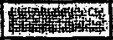 & 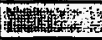 \\
\hline Day 3 & 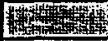 & (40) & 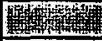 & 40 & 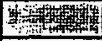 & 19 & 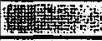 \\
\hline Day 4 & Fin & 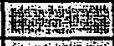 & 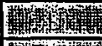 & 4 & 7 19 & 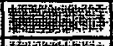 & 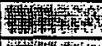 \\
\hline Day 5 & 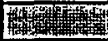 & 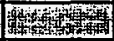 & 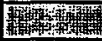 & 10 & 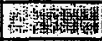 & 71) & 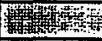 \\
\hline Day 6 & 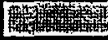 & (1) & 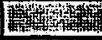 & 10 & In: & 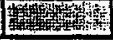 & 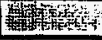 \\
\hline
\end{tabular}

Dissolved Oxygen (After Renewal)

\begin{tabular}{|c|c|c|c|c|c|c|c|}
\hline & \multicolumn{7}{|c|}{ Test Concentration } \\
\hline & Control & $6.0 \%$ & $12.0 \%$ & $25.0 \%$ & $50 \%$ & $100 \%$ & \\
\hline Initial & 8.6 & \begin{tabular}{|r|}
8.3 \\
\end{tabular} & 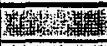 & 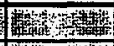 & 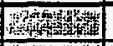 & 8.3 & \\
\hline Day 1 & 8.5 & Sry & 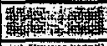 & A & Dy & 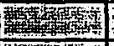 & 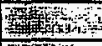 \\
\hline Day 2 & 8.6 & Bn & 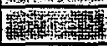 & (3) & 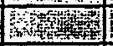 & 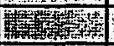 & Yhe \\
\hline Day 3 & 8.7 & 34t & 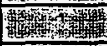 & 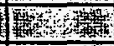 & 月14 & 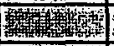 & Wh \\
\hline Day 4 & 8.6 & S小 & Hity & 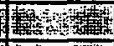 & 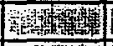 & 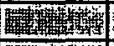 & 4y \\
\hline Day 5 & 8.6 & मn & 4 & 194 & 14 & 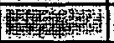 & 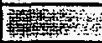 \\
\hline Day 6 & 8.7 & तियद्य & What & $\sqrt{4}$ & कियो & Hents & 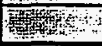 \\
\hline
\end{tabular}

Dilution Water \& Effluent Parameters

\begin{tabular}{|l|r|r|r|r|l|l|}
\hline & Res Cl. & Alkalin & Hard. & Cond. & & \\
\hline & $\mathrm{mg} / \mathrm{L}$ & $\mathrm{mg} / \mathrm{L}$ & $\mathrm{mg} / \mathrm{L}$ & $\mu \mathrm{mhos}$ & & \\
\hline Eff. Sample 1 & 0.02 & 18.5 & 6.0 & 63 & & \\
\hline Eff. Sample 2 & 0.06 & 14.4 & 4.0 & 46 & & \\
\hline Eff. Sample 3 & N/A & 8.4 & 24.0 & 30 & & \\
\hline & & & & & & \\
\hline Dilution Water & & 2.1 & 4.0 & 30 & & \\
\hline & & & & & & \\
\hline & & & & & & \\
\hline
\end{tabular}

\section{pH (Before Renewal)}

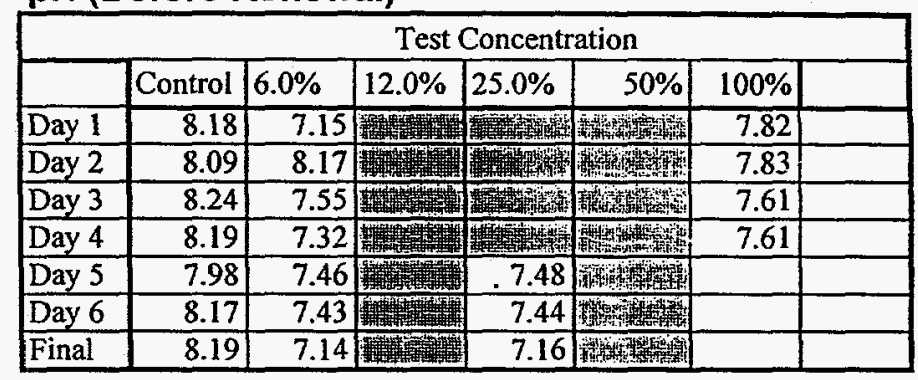

Dissolved Oxygen (Before Renewal)

\begin{tabular}{|c|c|c|c|c|c|c|c|}
\hline & \multicolumn{7}{|c|}{ Test Concentration } \\
\hline & Control & $6.0 \%$ & $12.0 \%$ & $25.0 \%$ & $50 \%$ & $100 \%$ & \\
\hline Day 1 & 8.2 & 7.9 & 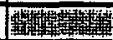 & 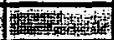 & 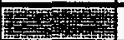 & 7.9 & \\
\hline Day 2 & 8.5 & 8.4 & 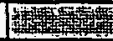 & 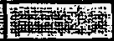 & H: & 8.3 & \\
\hline Day 3 & 8.5 & 8.4 & 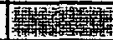 & 4 & Evat & 8.5 & \\
\hline Day 4 & 8.6 & & 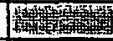 & 14 & III & & \\
\hline Day 5 & 8.5 & 8.0 & 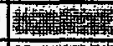 & W & (1) & 8.1 & \\
\hline Day 6 & 8.6 & 8.1 & W & wh & 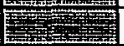 & 8.3 & \\
\hline Final & 8.6 & 8.5 & 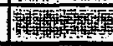 & 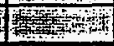 & Bin & 8.1 & \\
\hline
\end{tabular}

\section{Temperature}

\begin{tabular}{|l|r|}
\hline \multicolumn{2}{|c|}{ Incubator ${ }^{\circ} \mathrm{C}$} \\
\hline Initial & 24.2 \\
\hline Day 1 & 24.3 \\
\hline Day 2 & 24.3 \\
\hline Day 3 & 24.4 \\
\hline Day 4 & 25.1 \\
\hline Day 5 & 25.9 \\
\hline Day 6 & 24.5 \\
\hline Final & 24.4 \\
\hline
\end{tabular}




\section{Day Chronic Definitive Survival and Reproduction Bioassay \\ Method: EPA/600/4-89/001}

Test Organism:

Ceriodaphnia dubia

Facility:Westinghouse Savannah River Company

Sample ID:Outfall WE-001

\section{Date: $\quad$ 7/23/96}




\section{CHRONIC DEFINITIVE SURVIVAL AND REPRODUCTION TEST Survival and Reproduction Results}

Client: Westinghouse Savannah River Company

Sample Identification: Outfall WE-001

Test Date: $\quad 7 / 23 / 96 \quad$ T6184

\begin{tabular}{|c|c|c|c|c|c|c|c|}
\hline \multicolumn{3}{|c|}{ Reproduction at } & $0.0 \%$ & \multicolumn{5}{c|}{ Concentration } \\
\hline Rep. & Day 2 & Day 3 & Day 4 & Day 5 & Day 6 & Day 7 & Total \\
\hline 1 & 0 & 0 & 0 & 0 & 7 & 12 & 19 \\
\hline 2 & 0 & 0 & 4 & 6 & 0 & 8 & 18 \\
\hline 3 & 0 & 2 & 0 & 6 & 7 & 5 & 20 \\
\hline 4 & 0 & 0 & 0 & 7 & 10 & 13 & 30 \\
\hline 5 & 0 & 0 & 4 & 4 & 12 & 0 & 20 \\
\hline 6 & 0 & 0 & 3 & 8 & 0 & 14 & 25 \\
\hline 7 & 0 & 0 & 4 & 8 & 6 & 0 & 18 \\
\hline 8 & 0 & 0 & 3 & 7 & 0 & 12 & 22 \\
\hline 9 & 0 & 0 & 4 & 7 & 9 & 0 & 20 \\
\hline 10 & 0 & 0 & 0 & 8 & 11 & 10 & 29 \\
\hline
\end{tabular}

\begin{tabular}{|c|c|c|c|c|c|c|c|}
\hline \multicolumn{9}{|c|}{ Reproduction at } \\
\hline Rep. & Day 2 & Day 3 & Day 4 & Day 5 & Day 6 & Day 7 & Total \\
\hline 1 & 0 & 0 & 4 & 0 & 9 & 12 & 25 \\
\hline 2 & 0 & 0 & 4 & 6 & 10 & 0 & 20 \\
\hline 3 & 0 & 3 & 0 & 4 & 9 & 0 & 16 \\
\hline 4 & 0 & 0 & 3 & 6 & 8 & 0 & 17 \\
\hline 5 & 0 & 0 & 0 & 5 & 12 & 4 & 21 \\
\hline 6 & 0 & 0 & 4 & 6 & 0 & 15 & 25 \\
\hline 7 & 0 & 4 & 9 & 13 & 0 & 0 & 26 \\
\hline 8 & 0 & 2 & 0 & 0 & 13 & 16 & 31 \\
\hline 9 & 0 & 0 & 0 & 10 & 10 & 7 & 27 \\
\hline 10 & 0 & 0 & 3 & 8 & 0 & 15 & 26 \\
\hline
\end{tabular}

\begin{tabular}{|c|c|c|c|c|c|c|c|}
\hline \multicolumn{3}{|c|}{ Reproduction at } & $12.5 \%$ & \multicolumn{5}{|c|}{ Concentration } \\
\hline Rep. & Day 2 & Day 3 & Day 4 & Day 5 & Day 6 & Day 7 & Total \\
\hline 1 & 0 & 0 & 0 & 0 & 7 & 10 & 17 \\
\hline 2 & 0 & 0 & 3 & 10 & 11 & 0 & 24 \\
\hline 3 & 0 & 2 & 0 & 7 & 13 & 0 & 22 \\
\hline 4 & 0 & 0 & 3 & 4 & 7 & 7 & 21 \\
\hline 5 & 0 & 0 & 0 & 7 & 10 & 10 & 27 \\
\hline 6 & 0 & 0 & 5 & 7 & 0 & 9 & 21 \\
\hline 7 & 0 & 0 & 4 & 12 & 12 & 0 & 28 \\
\hline 8 & 0 & 0 & 4 & 0 & 16 & 15 & 35 \\
\hline 9 & 0 & 0 & 4 & 0 & 0 & 13 & 17 \\
\hline 10 & 0 & 2 & 0 & 8 & 6 & 0 & 16 \\
\hline
\end{tabular}

\begin{tabular}{|c|c|c|c|c|c|c|c|}
\hline \multicolumn{3}{|c|}{ Reproduction at } & $25.0 \%$ & \multicolumn{1}{c|}{ Concentration } \\
\hline Rep. & Day 2 & Day 3 & Day 4 & Day 5 & Day 6 & Day 7 & Total \\
\hline 1 & 0 & 0 & 0 & 0 & 0 & 15 & 15 \\
\hline 2 & 0 & 0 & 4 & 7 & 2 & 0 & 13 \\
\hline 3 & 0 & 3 & 7 & 0 & 14 & 0 & 24 \\
\hline 4 & 0 & 3 & 0 & 10 & 15 & 0 & 28 \\
\hline 5 & 0 & 0 & 4 & 8 & 5 & 5 & 22 \\
\hline 6 & 0 & 0 & 4 & 5 & 13 & 0 & 22 \\
\hline 7 & 0 & 5 & 0 & 3 & 12 & 0 & 20 \\
\hline 8 & 0 & 0 & 3 & 2 & 0 & 10 & 15 \\
\hline 9 & 0 & 0 & 4 & 0 & 13 & 10 & 27 \\
\hline 10 & 0 & 2 & 0 & 6 & 10 & 0 & 18 \\
\hline
\end{tabular}

\begin{tabular}{|c|c|c|c|c|c|c|c|}
\hline \multicolumn{6}{|c|}{ Reproduction at } & \multicolumn{7}{l|}{ Co\% } & \multicolumn{5}{|c|}{ Concentration } \\
\hline Rep. & Day 2 & Day 3 & Day 4 & Day 5 & Day 6 & Day 7 & Total \\
\hline 1 & 0 & 0 & 0 & 8 & 0 & 18 & 26 \\
\hline 2 & 0 & 0 & 5 & 14 & 10 & 0 & 29 \\
\hline 3 & 0 & 3 & 5 & 0 & 12 & 0 & 20 \\
\hline 4 & 0 & 0 & 0 & 8 & 13 & 4 & 25 \\
\hline 5 & 0 & 0 & 5 & 7 & 2 & 10 & 24 \\
\hline 6 & 0 & 0 & 5 & 8 & 0 & 12 & 25 \\
\hline 7 & 0 & 5 & 0 & 13 & 2 & 0 & 20 \\
\hline 8 & 0 & 0 & 4 & 7 & 0 & 17 & 28 \\
\hline 9 & 0 & 0 & 5 & 11 & 0 & 11 & 27 \\
\hline 10 & 0 & 0 & 4 & 7 & 0 & 16 & 27 \\
\hline
\end{tabular}

\begin{tabular}{|c|c|c|c|c|c|c|c|}
\hline \multicolumn{7}{|c|}{ Reproduction at } \\
\hline Rep. & Day 2 & Day 3 & Day 4 & Day 5 & Day 6 & Day 7 & Total \\
\hline 1 & 0 & 0 & 4 & 12 & 0 & 16 & 32 \\
\hline 2 & 0 & 0 & -4 & 10 & 14 & 0 & 28 \\
\hline 3 & 0 & 1 & 0 & 6 & 11 & 0 & 18 \\
\hline 4 & 0 & 0 & 0 & 10 & 8 & 13 & 31 \\
\hline 5 & 0 & 0 & 0 & 1 & 10 & 12 & 23 \\
\hline 6 & 0 & 0 & 3 & 8 & 0 & 14 & 25 \\
\hline 7 & 0 & 0 & 5 & 11 & 17 & 0 & 33 \\
\hline 8 & 0 & 3 & 4 & 0 & 11 & 0 & 18 \\
\hline 9 & 0 & 0 & 3 & 12 & 15 & 0 & 30 \\
\hline 10 & 0 & 0 & 4 & 3 & 0 & 12 & 19 \\
\hline
\end{tabular}

\footnotetext{
$X=$ Dead

$L=$ Lost or caught in surface tension of water
} 


\section{CHRONIC DEFINITIVE SURVIVAL AND REPRODUCTION TEST \\ Statistical Analyses}

Client: Westinghouse Savannah River Company

Sample Identification: Outfall WE-001

Test Date: $\quad$ 7/23/96

\section{Test for Normality}

Test Used: Shapiro Wilks

$\begin{array}{rr}\mathrm{W}= & 0.933 \\ \text { critical } & 0.93\end{array}$

The data are normal in distribution

Test for Differences in Reproduction

Test Type Used: $\quad$ t Test

\begin{tabular}{|l|r|r|r|r|r|r|r|}
\multirow{2}{*}{ Test } & \multicolumn{7}{|c|}{ Test Concentration } \\
\cline { 2 - 8 } & Control & $6.25 \%$ & $12.5 \%$ & $25.0 \%$ & $50 \%$ & $100 \%$ & \\
\hline$t$ Value & -0.59 & -0.32 & 0.77 & -1.36 & -1.64 & \\
\hline critical & 2.33 & 2.33 & 2.33 & 2.33 & 2.33 & \\
\hline mean & 22.1 & 23.4 & 22.8 & 20.4 & 25.1 & 25.7 & \\
\hline \multicolumn{2}{|l|}{$\mathrm{MSD}=$} & 5.13 & & &
\end{tabular}

NOEC (Survival): $100 \%$ effluent

NOEC (Reproduction): $100 \%$ effluent

\section{Test for Homogeneity of Variance}

Test Used: Bartlett's Test

$\mathrm{B}=$
critical

4.62

15.1

The data are homogeneous in variance

\section{Effluent Conc. vs. Reproduction}

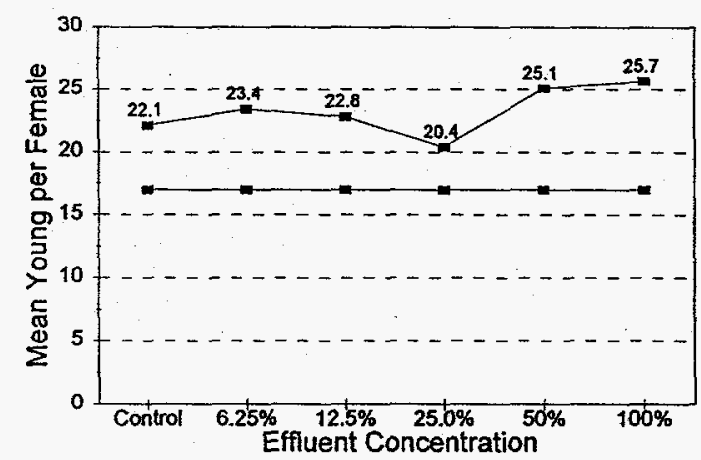

Summary

The effluent is not toxic 


\section{CHRONIC DEFINITIVE SURVIVAL AND REPRODUCTION TEST \\ Water Chemistry}

Client: Westinghouse Savannah River Company

Sample Identification: Outfall WE-001

Test Date: $\quad$ 7/23/96

pH (After Renewal)

\begin{tabular}{|c|c|c|c|c|c|c|c|}
\hline & \multicolumn{7}{|c|}{ Test Concentration } \\
\hline & Control & $6.25 \%$ & $12.5 \%$ & $25.0 \%$ & $50 \%$ & $100 \%$ & 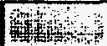 \\
\hline Initial & 6.51 & $3+4$ & 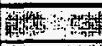 & 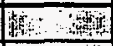 & कt? & 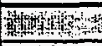 & 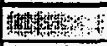 \\
\hline Day 1 & 6.52 & $2 \mathrm{~S}_{4} 4 \mathrm{~s}$ & 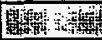 & 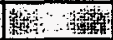 & Ix, and & 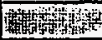 & 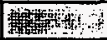 \\
\hline Day 2 & 6.51 & काt & 20 & Wy & 3y & 3 & 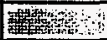 \\
\hline Day 3 & 6.47 & $1+4$ & Whan & An & 24 & W & 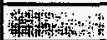 \\
\hline Day 4 & 6.53 & Butwe & at & $\sqrt{1+4}$ & Pfontw & 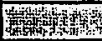 & 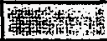 \\
\hline Day 5 & 6.59 & कि? & 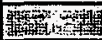 & 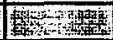 & 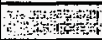 & The & 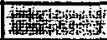 \\
\hline Day 6 & 6.49 & s.m & 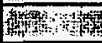 & 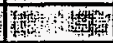 & $1 \mathrm{ntm}$ & 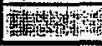 & 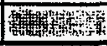 \\
\hline
\end{tabular}

\section{Dissolved Oxygen (After Renewal)}

\begin{tabular}{|c|c|c|c|c|c|c|c|}
\hline & \multicolumn{7}{|c|}{ Test Concentration } \\
\hline & Control & $6.25 \%$ & $12.5 \%$ & $25.0 \%$ & $50 \%$ & $100 \%$ & Mest \\
\hline Initial & 7.7 & 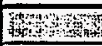 & 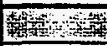 & 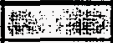 & ring & Ty & 14 \\
\hline Day 1 & 8.5 & DAs? & 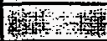 & 4 & D+4at & 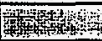 & 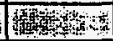 \\
\hline Day 2 & 7.8 & मि & min & $3 x-4$ & ता? & 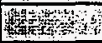 & 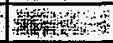 \\
\hline Day 3 & 8.1 & $1+4$ & w & 將 & $\therefore \quad 4$ & Mns & 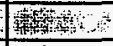 \\
\hline Day 4 & 8.1 & $\sqrt{1+4}$ & 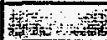 & 15, & 14 & Mys & W \\
\hline Day 5 & 8.6 & का4t & 繁霖 & (1) & 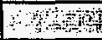 & 14 & 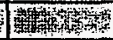 \\
\hline Day 6 & $\overline{8.4}$ & Bymb & (1) & 1) & 14ts & Whe & 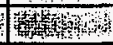 \\
\hline
\end{tabular}

\section{Dilution Water \& Effluent Parameters}

\begin{tabular}{|l|c|r|r|r|r|r|}
\hline & Res Cl. & Alkalin & Hard. & Cond. & & \\
\hline & $\mathrm{mg} / \mathrm{L}$ & $\mathrm{mg} / \mathrm{L}$ & $\mathrm{mg} / \mathrm{L}$ & $\mu \mathrm{mhos}$ & \\
\hline Eff. Sample 1 & 0.13 & 24.7 & 6 & 75.5 & & \\
\hline Eff. Sample 2 & $<.01$ & 28.8 & 6 & 82 & & \\
\hline Eff. Sample 3 & 0.07 & 26.8 & 6 & 74.5 & & \\
\hline & & & & & & \\
\hline Dilution Water & & 4 & 4 & 15 & \\
\hline & & & & & & \\
\hline & & & & & & \\
\hline
\end{tabular}

$\mathrm{pH}$ (Before Renewal)

\begin{tabular}{|c|c|c|c|c|c|c|c|}
\hline & \multicolumn{7}{|c|}{ Test Concentration } \\
\hline & Control & $6.25 \%$ & $12.5 \%$ & $25.0 \%$ & $50 \%$ & $100 \%$ & 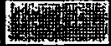 \\
\hline Day 1 & 7.69 & 7.25 & 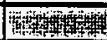 & tents & fantost & 7.64 & 40 \\
\hline Day 2 & 8.13 & 9.97 & 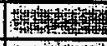 & Notres & 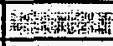 & 7.83 & 118 \\
\hline Day 3 & 7.79 & 7.58 & 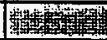 & What & pady & 7.65 & Btw-1, \\
\hline Day 4 & 7.96 & 7.67 & 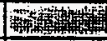 & mand & 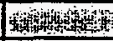 & 8.03 & -1) \\
\hline Day 5 & 7.63 & 7.49 & 篓 & Whan & 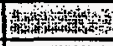 & 7.56 & 整 \\
\hline Day 6 & 7.88 & 7.63 & trits & 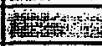 & 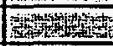 & 7.64 & (1) \\
\hline Final & 7.93 & 7.67 & 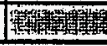 & 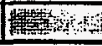 & 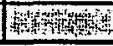 & 7.89 & $4+4$ \\
\hline
\end{tabular}

Dissolved Oxygen (Before Renewal)

\begin{tabular}{|c|c|c|c|c|c|c|c|}
\hline & \multicolumn{7}{|c|}{ Test Concentration } \\
\hline & Control & $6.25 \%$ & $12.5 \%$ & $25.0 \%$ & $50 \%$ & $100 \%$ & 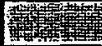 \\
\hline Day 1 & 8.4 & 8.4 & Pry & x+4 & Hotsts & 8.5 & W \\
\hline Day 2 & 7.8 & 7.9 & 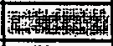 & 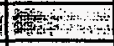 & Wentw & 7.8 & Hint \\
\hline Day 3 & 8.4 & 8.5 & 215 & 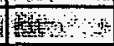 & का & 8.4 & (1) \\
\hline Day 4 & 8.0 & 8.3 & and & 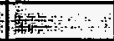 & Haph & 7.9 & Pat \\
\hline Day 5 & 8.7 & 8.5 & 5int & $y=$ & w & 8.7 & 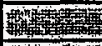 \\
\hline Day 6 & 8.7 & 8.5 & 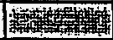 & 4 & thatsms & 8.4 & mix \\
\hline Final & 8.6 & 8.6 & 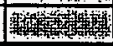 & 19y & 1964) & 8.5 & 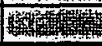 \\
\hline
\end{tabular}

\section{Temperature}

\begin{tabular}{|l|r|}
\hline \multicolumn{2}{|c|}{ Incubator ${ }^{\circ} \mathrm{C}$} \\
\hline Initial & 24.3 \\
\hline Day 1 & 24.5 \\
\hline Day 2 & 24.5 \\
\hline Day 3 & 25.0 \\
\hline Day 4 & 24.4 \\
\hline Day 5 & 24.5 \\
\hline Day 6 & 24.5 \\
\hline Final & 26.0 \\
\hline
\end{tabular}

